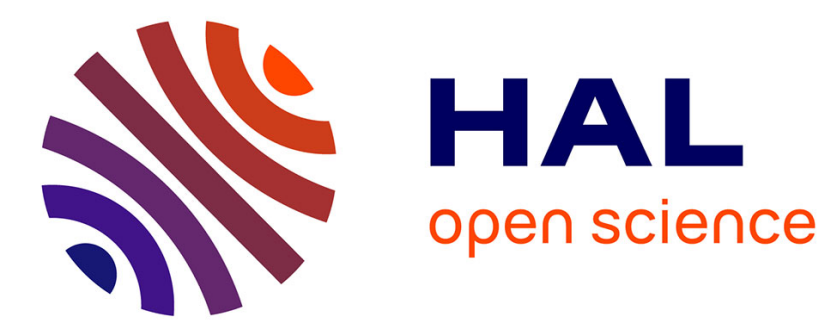

\title{
Challenges and Opportunities in Multicatalysis
}

Sebastian Andres Martinez Pena, Lukas Rainer Veth, Bruno Lainer, Pawel

Dydio

\section{To cite this version:}

Sebastian Andres Martinez Pena, Lukas Rainer Veth, Bruno Lainer, Pawel Dydio. Challenges and Opportunities in Multicatalysis. ACS Catalysis, 2021, 11 (7), pp.3891-3915. 10.1021/acscatal.0c05725 . hal-03281109

\section{HAL Id: hal-03281109 \\ https://hal.science/hal-03281109}

Submitted on 7 Jul 2021

HAL is a multi-disciplinary open access archive for the deposit and dissemination of scientific research documents, whether they are published or not. The documents may come from teaching and research institutions in France or abroad, or from public or private research centers.
L'archive ouverte pluridisciplinaire HAL, est destinée au dépôt et à la diffusion de documents scientifiques de niveau recherche, publiés ou non, émanant des établissements d'enseignement et de recherche français ou étrangers, des laboratoires publics ou privés. 


\title{
Challenges and Opportunities in Multi-Catalysis
}

\author{
Sebastián Martínezł, Lukas Vethł, Bruno Lainer, and Pawet Dydio* \\ University of Strasbourg, CNRS, ISIS UMR 7006, 8 allée Gaspard Monge, 67000 Strasbourg, \\ France
}

\begin{abstract}
Multi-catalysis is an emerging field targeting the development of efficient catalytic transformations to quickly convert relatively simple starting materials into more complex valueadded products. Within multi-catalytic processes either multiple catalysts execute single reactions or precise sequences of multiple catalytic reactions occur in a 'one-pot' fashion. Attractively, multi-catalytic protocols not only enable transformations that are inaccessible through classic approaches, but also are able to significantly reduce the time, waste, and cost of the synthetic processes, making organic synthesis more resources efficient. In this Perspective article, we review different strategies in multi-catalysis that bring distinct challenges and opportunities. We divide this overarching field into three main categories: cooperative, domino, and relay catalysis. Each category is described along with representative examples to highlight its features. Special emphasis is dedicated to relay catalysis, which is further discussed in its sub-categories. Lastly, we provide an analysis of systems that incorporate higher levels of complexity and further underscore the potential of multi-catalytic systems.
\end{abstract}

Keywords: multi-catalysis, cooperative catalysis, relay catalysis, orthogonal relay catalysis, sequential catalysis, auto-relay catalysis, assisted relay catalysis, complex systems chemistry 


\section{Introduction:}

The emergence of catalysis has had a transformative impact on various areas, including the production of chemicals, materials, and fuels, but also healthcare, agriculture, and the environment. ${ }^{1}$ From a historical standpoint, first catalysts were developed to enhance the performance of known reactions, but later catalysis started enabling new transformations to address new challenges of the time. Catalytic transformations are typically constrained to one catalyst enabling a single reaction. However, the field continues evolving in complexity to further increase its potential. ${ }^{2-19}$ Recently, there has been growing interest in developing multi-catalytic systems, which are inspired by the complexity of catalytic reactions occurring in nature. ${ }^{20,21}$ Such efforts are further driven by remarkable characteristics of biosynthesis, that is, excellent selectivity, high yields, and material efficiency when producing complex molecules directly without any isolation of intermediates. However, to develop such multi-catalytic systems, major challenges need to be addressed, including incompatibility between multiple catalysts or reagents, precise ordering of all individual processes, and operation of all reactions under a single set of conditions.

To shed light on different approaches that address the challenges and take advantage of the opportunities of multi-catalysis, we analyze the field and provide insights into its three main categories: cooperative, domino, and relay catalysis. Each type of multi-catalysis is illustrated with a selection of representative reports that highlight their different features. Special emphasis is paid to relay catalysis, sub-categories of which are discussed and further portrayed with corresponding examples. Lastly, more complex systems, consisting of combinations of main multi-catalytic categories, are reviewed. The overall goal of this Perspective article is to emphasize the vast potential of multi-catalytic 'one-pot' processes to further inspire development and promote applications of such processes across molecular sciences. 
Noteworthy, domino catalysis that uses a single catalyst to execute a series of reactions within one catalytic cycle lays at the edge of multi-catalysis, and as such, it might be considered to be outside of the field. However, we included domino catalysis into the scope of this Perspective article purposefully, in the efforts of portraying the full picture of synthetic systems that execute multiple catalytic events in one-pot.

Because multi-catalytic systems based solely on enzymatic catalysis encounter distinct challenges than the multi-catalytic systems incorporating 'small-molecule' catalysts, they stay beyond the scope of this article. We refer the readers to the corresponding reviews. ${ }^{22-25}$

\section{Definitions and classification}

The classification of different types of the multi-catalytic one-pot processes is somewhat inconsistent in the chemical literature. The herein proposed classification is inspired by the efforts of Fogg and Santos, ${ }^{11}$ and Patil ${ }^{5}$ and aims to provide a systematic analysis that includes all current approaches in this field. All systems with either multiple catalysts or multiple catalytic reactions taking place in the same reaction vessel are accounted for, irrespectively if all reagents or catalysts are present from the start or are added during the reaction along with the possible change of reaction conditions.

Our primary classification of multi-catalytic systems takes into account (i) how the catalysts operate, i.e., the number of catalytic cycles that are involved and if the catalytic cycles are intertwined, and (ii) the number of independent catalytic reactions, i.e., number of bonds being formed, modified, or broken in the overall process, leading to the three categories of "cooperative catalysis", "domino catalysis", and "relay catalysis". Because relay catalysis encompasses a range of distinct systems bearing different challenges and opportunities, the further division into subcategories is proposed. Here, the distinction is based on whether one or multiple pre-catalysts are 
used, i.e., whether each pre-catalyst operates in single or multiple independent catalytic cycles, and whether a change in reaction conditions or an addition of components throughout the process takes place. The general features for each category and sub-category are summarized in Figure 1.

a. Cooperative Catalysis

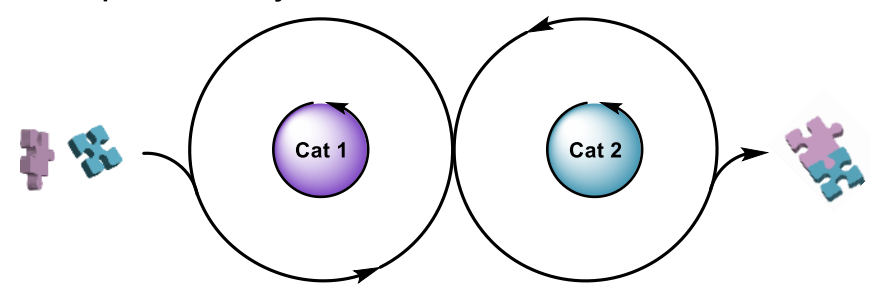

Key features:

Number of catalytic reactions ${ }^{a}$ in the process: 1

Number of catalysts in the process: $\geq 2$

Number of catalytic cycles in the process: $\geq 2$

Shared intermediates between catalytic cycles: yes b. Domino Catalysis

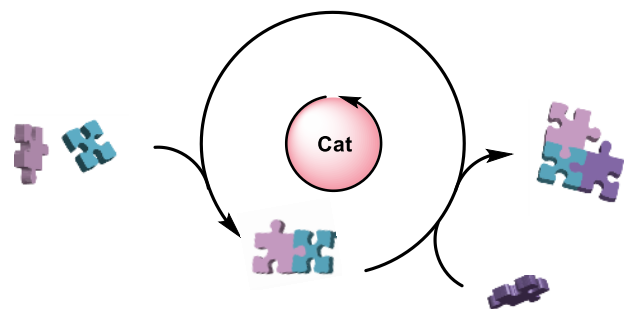

Key features:

Number of catalytic reactions ${ }^{a}$ in the process: $\geq 2$

Number of catalysts in the process: 1

Number of catalytic cycles in the process: 1

Shared intermediates between catalytic cycles: not applicable

\section{c. Relay Catalysis}

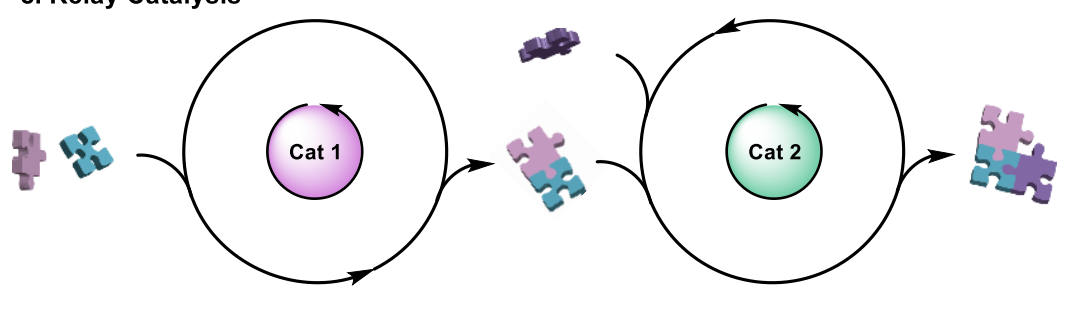

Subcategories of Relay Catalysis:

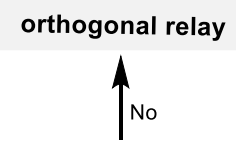

Change of conditions?

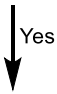

sequential relay

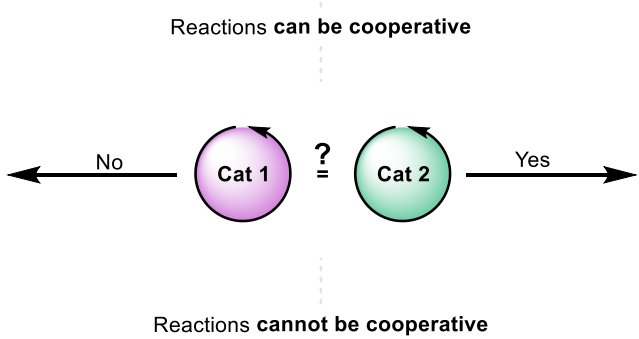

Key features:

Number of catalytic reactions ${ }^{a}$ in the process: $\geq 2$

Number of catalysts in the process: $\geq 1$

Number of catalytic cycles in the process: $\geq 2$

Shared intermediates between catalytic cycles: No

${ }^{a}$ A single catalytic reaction understood as a distinct event of making, modifying, or breaking a bond occurring without any non-catalytic reaction intermediates

Figure 1. General characteristics of different categories and subcategories of multi-catalytic onepot processes discussed in the Perspective article. 
It is noteworthy that the literature often refers to some multi-catalytic processes as "cascade catalysis". Because of its wide-spread use in different independent contexts, we propose the term "cascade catalysis" to be avoided in the future, as previously also suggested by Tietze. ${ }^{26}$

We also need to note that for the sake of simplicity in this article, we use the term 'catalyst' rather liberally to indicate a molecule that is either catalytically active or itself forms a catalytically active species under reaction conditions. When necessary for clarity, we differentiate between a 'pre-catalyst' and an actual 'catalyst' or 'catalytic species'.

\section{Cooperative catalysis}

Cooperative catalysis, often referred to as synergistic catalysis, ${ }^{16}$ refers to catalytic systems in which multiple catalysts operate in concert by sharing their individual catalytic cycles in a single reaction to create, modify, or break a single bond. Consequently, the accumulation of reaction

intermediates is not possible. The field of research has been extensively reviewed recently. ${ }^{18,27-29}$ Therefore, below we only discuss representative examples that indicate the key features enabled by cooperative catalysis, noting all possible combinations regarding the different types of catalysts, such as organo/organo, organo/metal, and metal/metal, found in dual-catalytic systems. It is worth noting that in a recent perspective article on enantioselective synthesis with the aid of cooperative dual-catalytic systems, ${ }^{17}$ Hoveyda and co-workers pointed out that the two catalysts are not necessarily acting cooperatively in such reactions. Therefore, the term cooperative catalysis might be somewhat misleading.

\section{Enabling precise stereocontrol}

In stereoselective synthesis, the synergistic action of two catalysts enables the enhancement of stereoselectivity control through the formation of highly organized transition states. For instance, Carreira $^{30}$ and co-workers developed a metal/organo dual-catalytic system for the enantio- and 
diastereodivergent $\alpha$-allylation of branched aldehydes $\mathbf{1 . 1}$ to form $\gamma, \delta$-unsaturated aldehydes $\mathbf{1 . 3}$ from a pair of racemic starting materials (Scheme 1). The reaction occurs in the presence of a chiral Ir-phosphoramidite complex that activates allylic alcohol 1.2 to form allyliridium intermediate $\mathbf{1 . 4}$ and chiral cinchona alkaloid A1 or A2 that activates aldehyde $\mathbf{1 . 1}$ through the formation of an iminium intermediate $\mathbf{1 . 5}$. Because each catalyst controls the selectivity of each new stereogenic center independently, each of all four possible stereoisomers of $\mathbf{1 . 3}$ can be prepared selectively by selecting a pair of suitable enantiomers of both catalysts. By adapting the strategy, the same group subsequently reported two similar systems for the $\alpha$-allylation of linear aldehydes, ${ }^{31}$ and protected $\alpha$-amino or $\alpha$-hydroxyacetaldehydes. ${ }^{32}$ The strategy was also utilized in a number of other studies with metal/metal ${ }^{33-39}$, metal/organo, ${ }^{40-42}$ and organo/organo ${ }^{43}$ dual-catalytic systems, which demonstrated that multi-catalysis provides powerful tools for stereodivergent asymmetric synthesis. 
Scheme 1. Ir-/chiral amine-catalyzed cooperative enantio- and diastereodivergent $\alpha$-allylation of $\alpha$-branched aldehydes

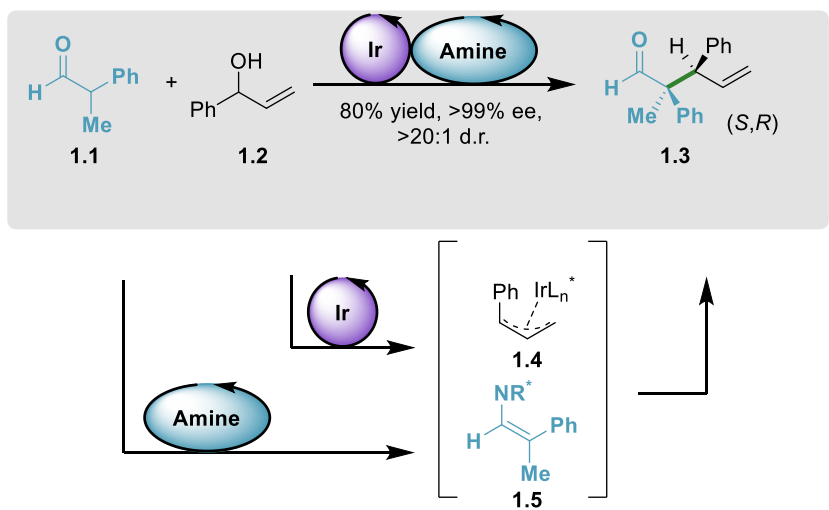

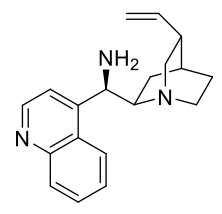

A1
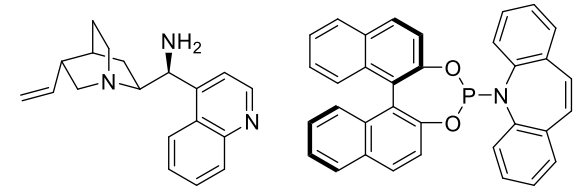

$(R)-\mathrm{L}$

$\begin{array}{ll}\text { Key feature: } & \\ \text { Ir } & =[\operatorname{lr}(\mathrm{COD}) \mathrm{Cl}]_{2}+(R / S)-\mathrm{L}\end{array}$




\section{Enabling new reaction pathways}

Thanks to the interactions between multiple catalytic cycles, multi-catalysis can create new reaction pathways, enabling transformations that are otherwise either kinetically or thermodynamically unfeasible. For instance, cross-coupling reactions involving alkyl boronic acids occurring in the presence of a single catalyst are typically sluggish, because transmetalation involving $\mathrm{sp}^{3}$-hybridized organoboronic acid $\mathbf{2 . 1}$ is rather slow and requires harsh reaction conditions, limiting the overall applicability. To address these issues, Molander ${ }^{44}$ and co-workers developed a metal/metal dual-catalytic system that combines a classical Ni-catalyst with an Irphotoredox catalyst (Scheme 2). ${ }^{45}$ The latter mediates a single-electron transfer (SET) to readily form benzyl radicals $\mathbf{2 . 4}$ from $\mathbf{2 . 1}$, which undergo fast single electron transmetalation with the $\mathrm{Ni}$ catalyst. Overall, incorporation of a SET enables efficient cross-coupling between $\mathrm{sp}^{3}$-centered potassium alkoxyalkyl- or benzyltrifluoroborates $\mathbf{2 . 1}$ and aryl bromides $\mathbf{2 . 2}$ under mild conditions and consequently for a broad range of substrates. In turn, the merger of photoredox catalysis and transition metal catalysis was examined by MacMillan and Doyle to execute cross-coupling reactions with alkyl carboxylic acids as coupling partners. ${ }^{46}$ The versatility of the approach was further indicated in other studies, including the report of Molander ${ }^{47}$ on using alkyl silicon derivatives as coupling partners and the work of Nishibayashi ${ }^{48}$ on using alkyl dihydropyridines as coupling partners. The field of cross-coupling reactions exploiting the combination of photoand transition metal-catalysts was recently reviewed. ${ }^{49}$ 
Scheme 2. Ir-/Ni-catalyzed cooperative cross-coupling of $\mathrm{sp}^{3}$-nucleophiles with aryl bromides assisted by a single-electron transmetalation step

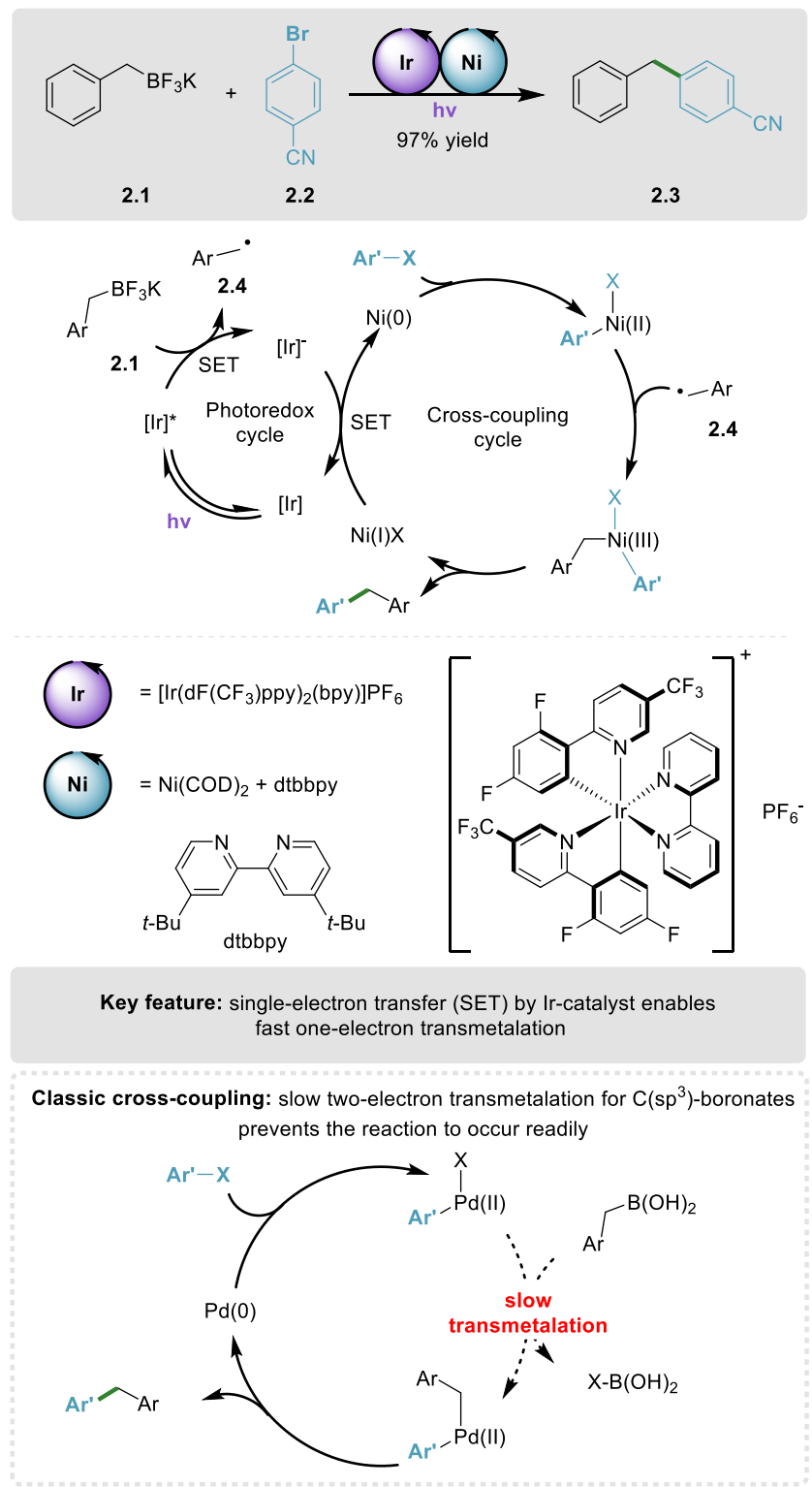

The combination of a photoredox mediated hydrogen atom transfer (HAT) with transition-metal catalysis proved attractive for functionalization of C-H bonds. ${ }^{50-54}$ In a notable example, MacMillan and coworkers ${ }^{55}$ established challenging arylation reactions of aliphatic substrates at their strong $\mathrm{C}\left(\mathrm{sp}^{3}\right)$-H bonds with aryl bromides occurring in the presence of a metal/metal dual- 
catalytic system (Scheme 3). In the reaction, a light-driven excitation of a decatungstate HAT photocatalyst mediates a hydrogen atom abstraction from alkyl nucleophile $\mathbf{3 . 1}$ to form nucleophilic carbon-centered radical 3.4, which is in turn captured by a $\mathrm{Ni}^{0}$-catalytic species forming a $\mathrm{Ni}^{\mathrm{I}}$-alkyl species. The latter undergoes sequential oxidative addition of aryl halide $\mathbf{3 . 2}$ to furnish a $\mathrm{Ni}^{\mathrm{III}}$ (aryl)(alkyl) species followed by subsequent reductive elimination affording the cross-coupled product 3.3. Lastly, a series of electron transfer events regenerates catalytic species, closing both catalytic cycles. Other reports on functionalization of unactivated $\mathrm{C}-\mathrm{H}$ bonds occurring in the presence of analogous metal/metal dual-catalytic systems further underscored the efficacy of the strategy. ${ }^{56-58}$

Scheme 3. W-/Ni-catalyzed arylation of strong $\mathrm{sp}^{3}-\mathrm{C}-\mathrm{H}$ bonds with aryl bromides enabled by light-driven hydrogen atom transfer (HAT)

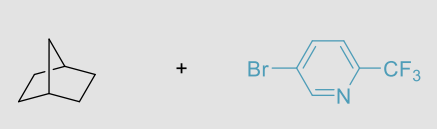

3.1

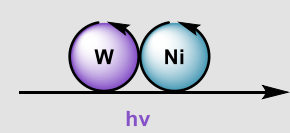

$61 \%$ yield

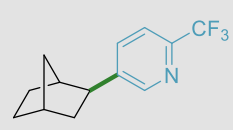

3.3
Key feature:
light-driven hydrogen atom transfer enables the in situ generation of strong radical nucleophiles for nickel-catalyzed crosscoupling with aryl electrophiles
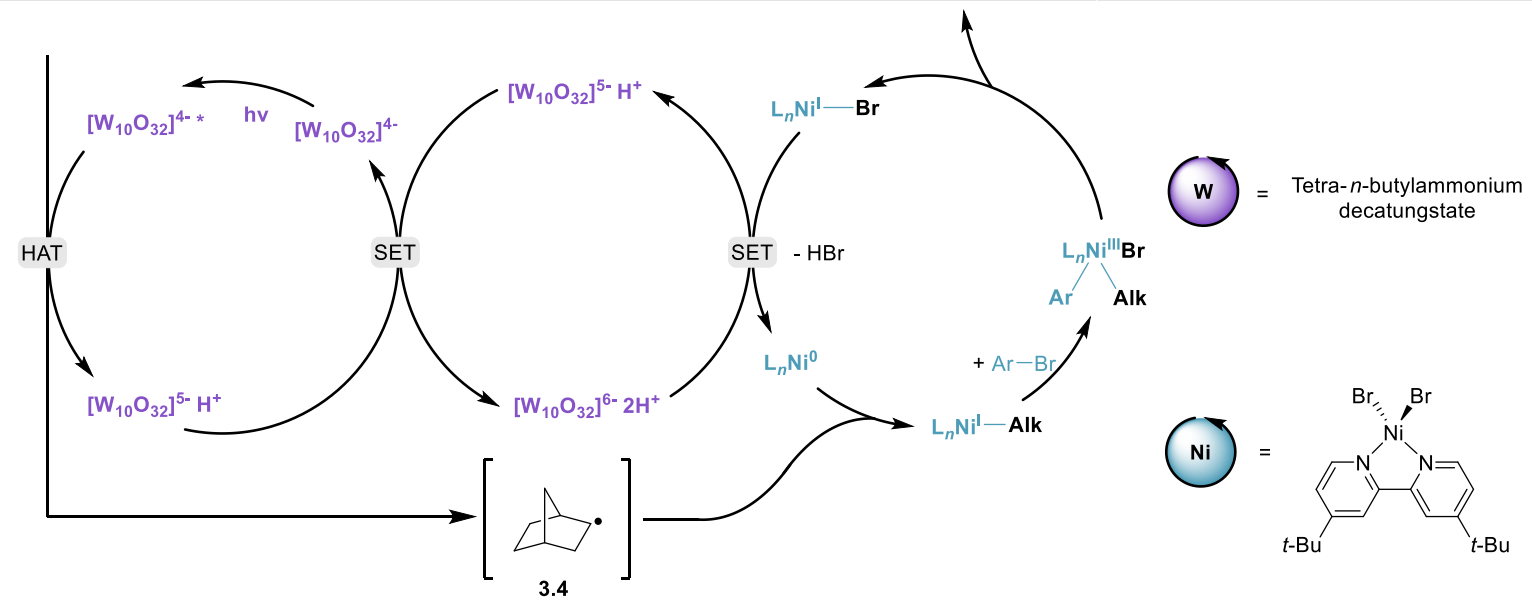

Cooperation between a photocatalyst (PC) and a hydrogen atom transfer (HAT) catalyst provided new opportunities for free radical organic chemistry. ${ }^{59}$ In general, open-shell radical intermediates can be readily generated by an electron transfer process between PCs and organic 
starting materials. Such radicals can be engaged in transformations that are inaccessible for the closed-shell starting materials. Upon completing the target reactions, the radical intermediates are converted back to the closed-shell products by reacting with the HAT catalysts. ${ }^{60}$ For instance, Nicewicz and coworkers ${ }^{61}$ recently established anti-Markovnikov hydrohalogenation of styrenyl alkenes occurring selectively in the presence of a organo/organo dual-catalytic system built of an acridinium salt acting as a PC and a thiophenol derivative acting as a HAT catalyst (Scheme 4). Initially, the visible light-excited photoredox catalyst reacts with alkene 4.1 through photoinduced electron-transfer (PET) to form alkene radical cation 4.4. The latter reacts with a chloride anion through the nucleophilic addition to produce benzylic carbon-centered radical 4.5. Upon a HAT from the redox-active thiol, the anti-Markovnikov hydrohalogenation product 4.3 is formed. Lastly, a source of acidic protons converts thiolate to the active hydrogen atom donor, allowing turnover of the HAT catalyst. Notably, several halogen, phosphate, and sulfonate anions can be used as nucleophiles, when a well-paired combination of PC and HAT catalysts is used. 
Scheme 4. Merging two organocatalysts enables the light-driven anti-Markovnikov hydrohalogentaion of styrenyl alkenes
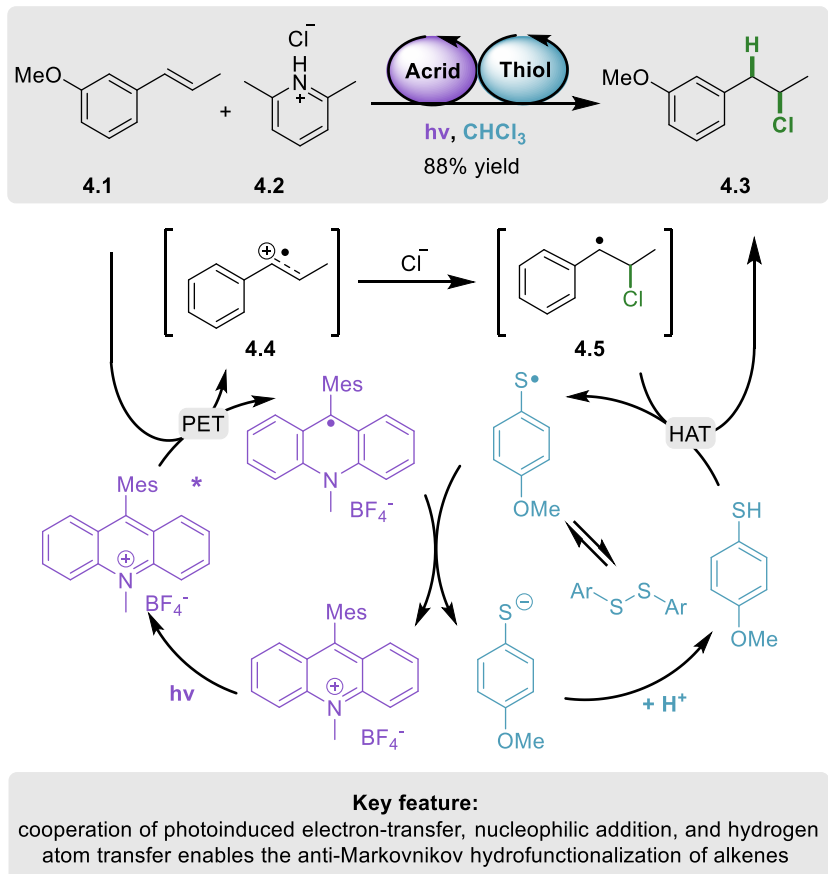

The abovementioned photocatalytic strategy using organocatalysts has been extensively studied to afford a range of new reactions, including recent studies on generation and trapping of alkene radical cations ${ }^{62}$ in other transformations such as hydroacetoxylation, ${ }^{63}$ intra- ${ }^{64}$ and intermolecular ${ }^{65}$ hydroamination, a range of novel polar radical crossover cycloadditions, ${ }^{66-68}$ synthesis of polysubstituted aldehydes, ${ }^{69}$ as well as a site-selective arene C-H amination ${ }^{70}$ and direct arene $\mathrm{C}-\mathrm{H}$ fluorination. ${ }^{71} \mathrm{HAT}$ organocatalysts, such as zwitterionic triazolium amidates, proved also effective in the organo/metal dual-catalytic systems enabling $\mathrm{C}-\mathrm{H}$ bond alkylation reactions. $^{72}$

\section{Enabling thermodynamically unfeasible transformations}

The ability to directly convert a racemic mixture of a compound into its enantiomerically pure form would be attractive from a synthetic standpoint. However, such transformations are 
thermodynamically unfeasible, due to the prospective increase in entropy $(\Delta \mathrm{G}=+0.42 \mathrm{kcal} / \mathrm{mol}){ }^{73}$ To overcome the intrinsic challenges of unfavorable thermodynamics, Miller, Knowles, ${ }^{73}$ and coworkers developed an elegant protocol of a light-driven direct deracemization of cyclic ureas $\mathbf{5 . 1}$ by the cooperative action of three catalysts namely an (achiral) Ir-photocatalyst, a chiral phosphate catalyst, and a chiral peptide thiol catalyst. The reaction proceeds through the excited-state redox events enabling a kinetic enrichment of one enantiomer (Scheme 5). First, both enantiomers of cyclic urea 5.1 undergo continuous reversible oxidation by the excited state of the Ir-photocatalyst to form a pair of transient chiral radical cations 5.3 and 5.4. Then, one enantiomer of the transiently formed $\mathbf{5 . 3}$ or $\mathbf{5 . 4}$ is preferentially deprotonated by the chiral phosphate catalyst with $86: 14$ selectivity. In turn, thus-formed $\alpha$-amino radical $\mathbf{5 . 5}$ undergoes enantioselective hydrogen atom transfer (HAT) in the presence of the chiral thiol catalyst, recovering preferentially the opposite enantiomer of the starting material with 79:21 selectivity. Upon reaching the steady-state level of optical enrichment, the effective overall deracemization is achieved with 96:4 er through the composition of selectivities of chiral oxidation (86:14 er) and chiral hydrogen atom transfer (79:21 er) steps. Overall, the multi-catalytic strategy enables to create the out-of-equilibrium state at the expense of light energy as the fuel. 
Scheme 5. Ir-/phosphate-/peptide thiol-catalyzed light-driven cooperative deracemization of ureas through stereoselective excited-state processes
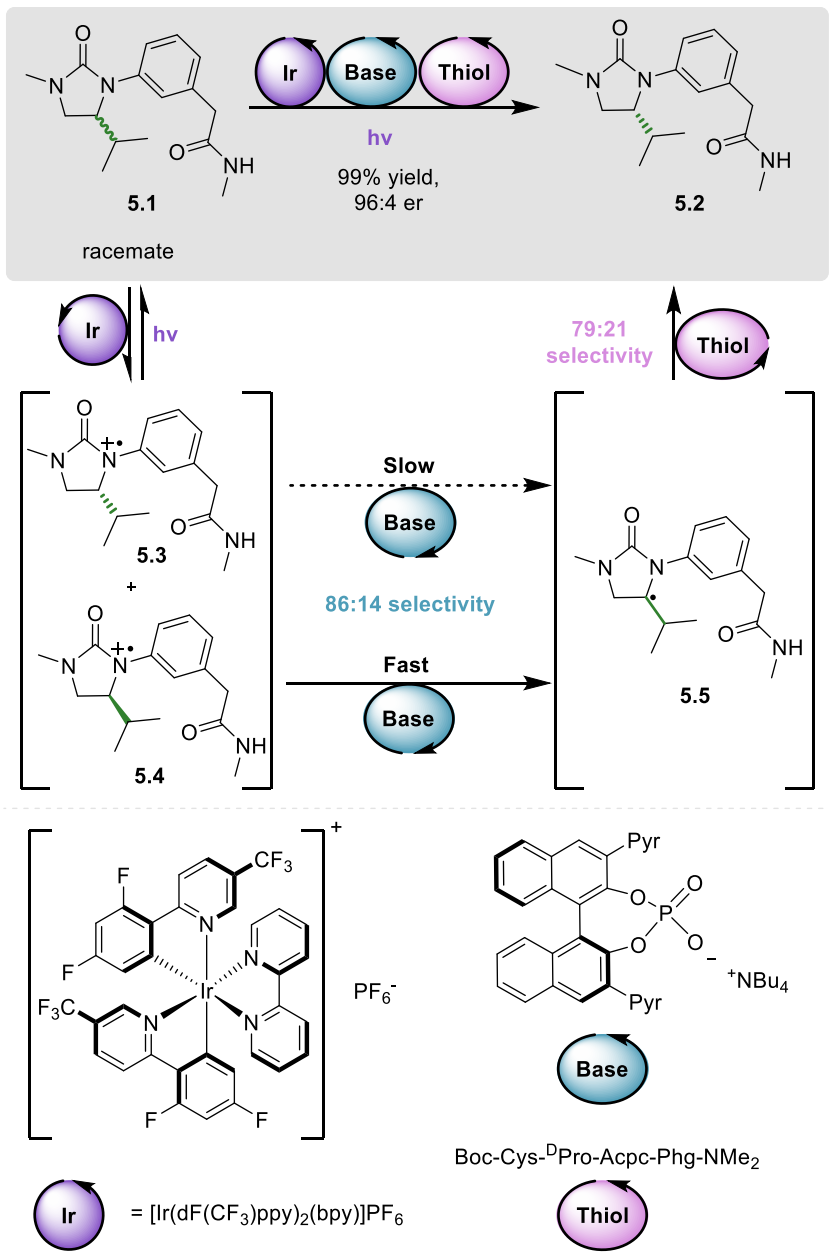

Boc-Cys- ${ }^{\text {P Pro-Acpc-Phg-NMe }}{ }_{2}$

(Thiol)

Cooperation between a series of light-driven organocatalytic processes furnished a kinetically controlled site-selective epimerization of natural sugars to afford rare sugar isomers, as recently reported by Wendlandt ${ }^{74}$ and co-workers (Scheme 6). Within the reaction, four organo-catalysts operate in concert, namely a photocatalyst (4-CzIPN), a hydrogen-atom abstraction catalyst (quinuclidine), a HAT catalyst (1-adamantane thiol), and a base (tetrabutylammonium pchlorobenzoate). First, the blue LED light-excited photocatalyst is quenched by quinuclidine to 
generate a quinuclidinium radical cation. The latter mediates an irreversible hydrogen-atom abstraction from substrate $\mathbf{6 . 1}$ to form sugar radical $\mathbf{6 . 3}$, followed by a diastereoselective HAT from the thiol catalyst to afford the epimerized sugar product $\mathbf{6 . 2}$ and the thiyl radical. A series of proton, electron, and hydrogen-atom transfers regenerates catalysts, closing the catalytic cycles.

Scheme 6. Cooperative metal-free quadruple-catalytic catalysis enables site-selective epimerization of sugars to form rare sugar isomers

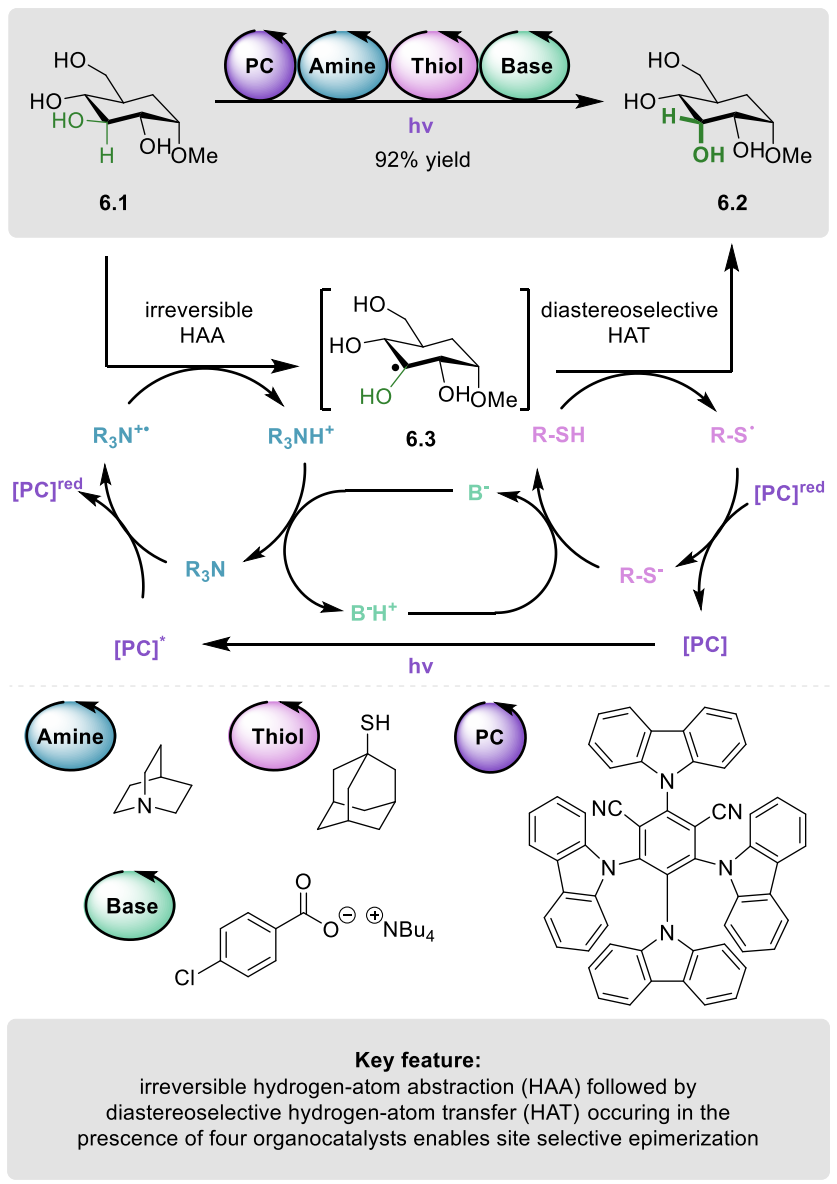




\section{Domino catalysis}

Domino catalysis refers to catalytic systems with typically one catalyst operating in one catalytic cycle to form, modify, or break multiple bonds, i.e., multiple distinct reactions take place within the same catalytic cycle. Considering that a catalyst typically remains bound to the intermediates until the sequence is completed, the accumulation of reaction intermediates is not possible. Because domino reactions have been extensively reviewed in journal articles ${ }^{75-80}$ and books, ${ }^{26,81}$ below we only discuss representative examples and we further refer the reader to the review literature. However, it is worth noting that some of the reviews do not strictly follow the same definition, sometimes using the term domino catalysis in a much broader sense.

It should be noted that domino catalysis, featuring only one catalyst that operates in one catalytic cycle, might not belong strictly to the field multi-catalysis. However, the approach represents a stepping stone towards the development of time- and resource-efficient processes with multiple catalytic bond-forming events occurring in one pot. Therefore, we consider its inclusion and a discussion of selected examples as pertinent for this Perspective article.

\section{Creating multiple bonds within a single linear substrate}

Domino reactions were shown to be particularly attractive for natural product synthesis, directly converting relatively simple linear starting materials into complex molecules with high diastereoand enantioselectivity. The substrates for such processes usually require a high level of design and preorganization for a transformation to be successful; however, the reported examples demonstrate the impressive increase of molecular complexity within a single synthetic step. For instance, Trost and co-workers ${ }^{82}$ employed an intramolecular Pd-catalyzed polyenyne cycloisomerization as the centerpiece of the total synthesis of tremulanes, a group of sesquiterpenes. A linear polyenyne starting material 7.1 containing all the carbon and oxygen atoms in the targeted places 
undergowent a sequence of reactions through a series of sequential hydropalladation, carbopalladation, and $\beta$-hydride elimination steps (Scheme 7). Overall, the Pd-catalyzed domino process resulted in the formation of 7.2 with three new stereocenters, one 5-membered, and one 7membered ring with high diastereoselectivity and yield in a single synthetic step. In an earlier work from the same group, ${ }^{83}$ a Pd-catalyzed polyenyne cycloisomerization led to the formation of six new C-C bonds and seven 5-membered rings, highlighting the synthetic capacity of domino catalysis.

Scheme 7. Pd-catalyzed intramolecular domino polyenyne cycloisomerization for the synthesis of terpene derivatives
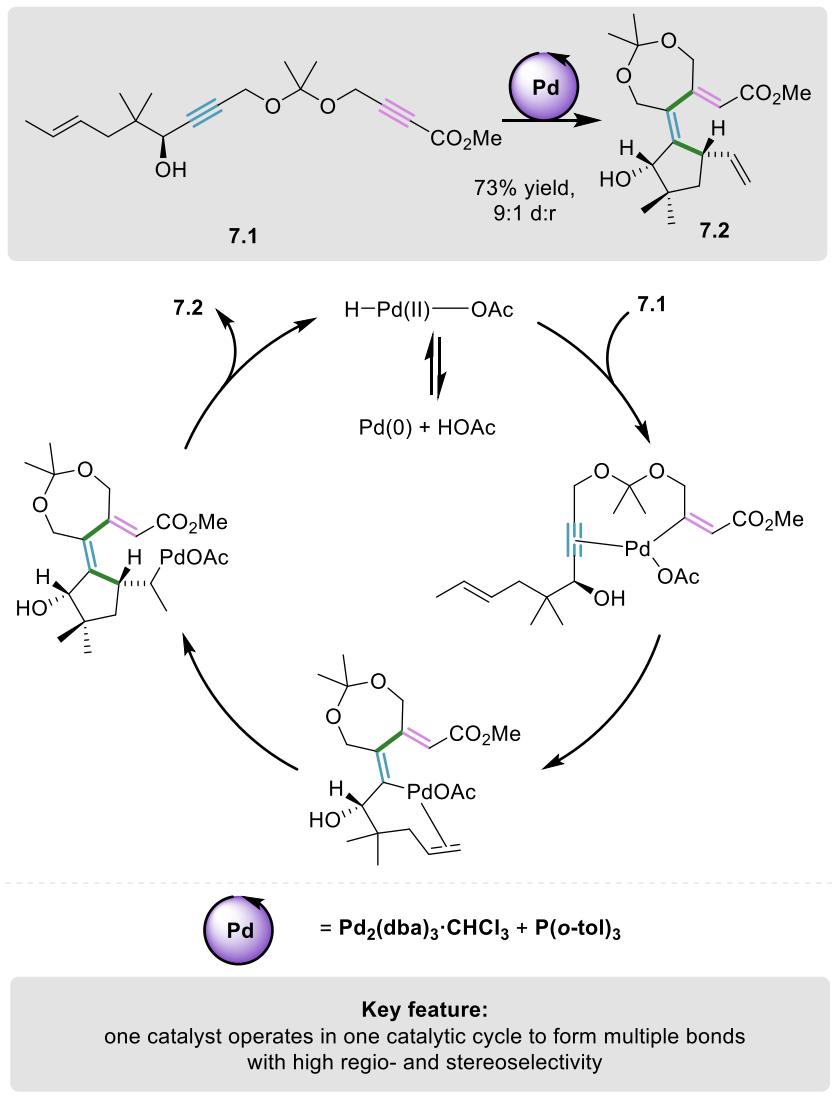


\section{Creating several bonds between multiple substrates}

Multiple building blocks can undergo intermolecular reactions to build up molecular complexity under domino catalysis protocols. For instance, Lautens and co-workers ${ }^{84}$ recently reported a Cocatalyzed domino transformation, which most likely occurs through a $\mathrm{C}-\mathrm{H}$ activation of pyridylindole $\mathbf{8 . 2}$ followed by a series of insertion steps involving 1,6-enyne $\mathbf{8 . 1}$ as the second starting material (Scheme 5). Overall, a pair of simple achiral starting materials are directly converted into pyrrolidine derivatives 8.3 with high regio- and diastereoselectivities and in high yields.

Scheme 8. Co-catalyzed enantioselective hydroarylation of 1,6-enynes through an intermolecular domino reaction

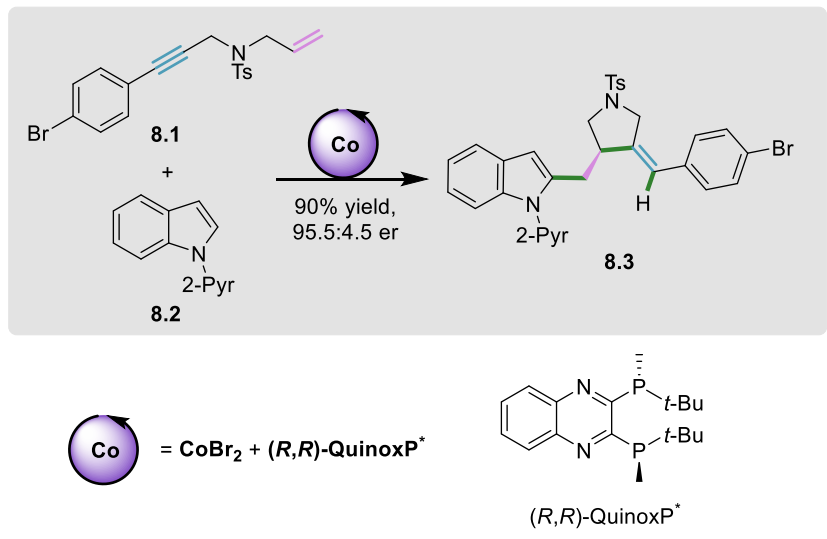

Key feature:
domino catalysis enables to form multiple bonds with high selectivity between multiple starting materials

\section{Relay catalysis}

Relay catalysis refers to catalytic transformations occurring in the presence of catalysts, which operate in multiple functionally distinct, non-interfering catalytic cycles to execute a sequence of independent reactions. Importantly, unlike for cooperative catalysis, here catalytic cycles do not share any catalytic intermediates. Therefore, the accumulation of intermediates of the sequence 
might be possible. It is worth noting that relay catalysis involving two catalysts is often referred to as tandem catalysis.

As discussed above, relay catalysis covers a range of distinct systems, which we further divided into sub-categories, depending on the number of catalysts and the reaction conditions. In the following chapters, we discuss different types of relay catalysis, exemplified by selected examples. Special emphasis is placed on the potential and challenges related to each of the sub-categories.

\subsection{Orthogonal relay catalysis}

Orthogonal relay catalysis refers to catalytic systems exploiting at least two catalysts, which are present and active from the start, with each catalyst mediating only one of multiple consecutive reactions. The strategy requires to address all the common challenges of multi-catalysis, which include (i) the compatibility between all catalysts and reagents, i.e., catalysts and reagents must not inhibit each other or undergo non-productive side reactions; (ii) common conditions, i.e., all reactions need to occur under the same set of reaction conditions; and (iii) reaction ordering, i.e., each catalyst is designed to undergo a selective reaction only with one specific intermediate of the reaction sequence. Despite all the requirements associated, orthogonal relay catalysis represents arguably an ideal case of relay catalysis, providing numerous features inaccessible in other cases. The representative examples that highlight such features are discussed below.

\section{$\underline{\text { Decreasing overall reaction time for two-step processes }}$}

Among typical advantages of orthogonal relay processes are reduction of the reaction time, the amount of work, and resources needed for the overall transformation when compared to the corresponding two-step process. For instance, a large volume of linear aliphatic alcohols in the industry is prepared by a two-step sequence involving hydroformylation of alkenes $\mathbf{9 . 1}$ to form aldehydes 9.3, which are later hydrogenated to the target alcohol products 9.2. To improve the 
economy of the process by limiting the overall reaction time of the process, Nozaki and coworkers $^{85,86}$ developed the orthogonal relay of hydroformylation and hydrogenation steps occurring in parallel in the same vessel in the presence of a dual-catalytic Rh-/Ru-system (Scheme 9). The control experiments confirmed that both reactions are fully independent of each other. The overall yield remains the same, irrespectively whether the process is conducted in a one-step or two-step fashion, with the catalysts added sequentially.

Scheme 9. Rh-/Ru-catalyzed orthogonal relay of hydroformylation-hydrogenation of terminal alkenes to form terminal alcohols

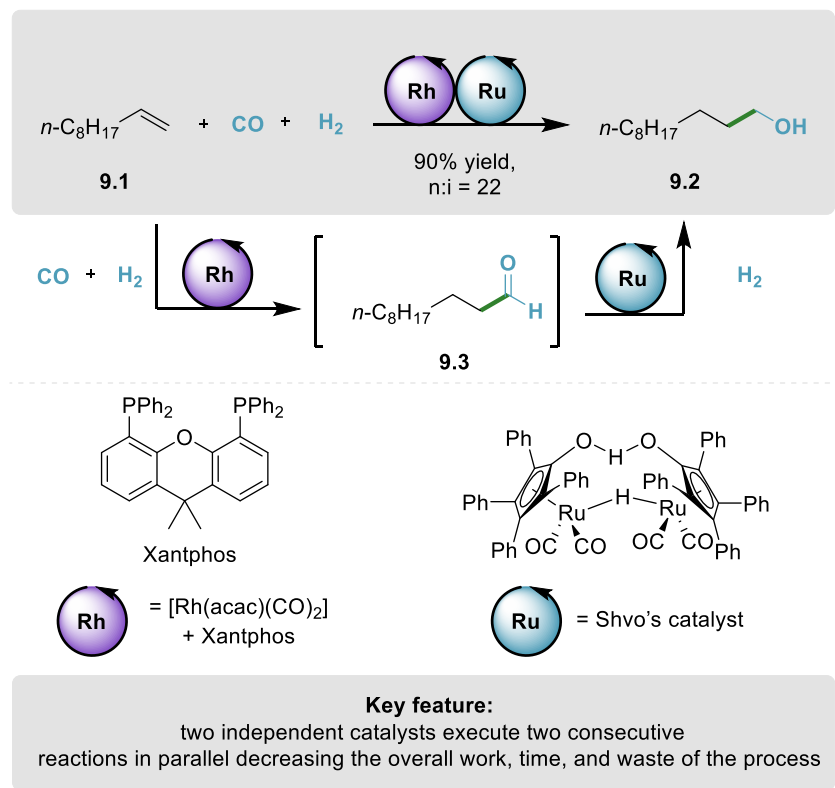

Enabling selective sequences under mild conditions

Hydroaminomethylation, a formal combination of hydroformylation of alkenes with reductive amination of aldehyde intermediates, represents an attractive strategy to convert abundant alkenes into value-added aliphatic amines. Beller and co-workers ${ }^{87}$ reported that the full sequence can be executed in the presence of a single Rh-complex (auto-relay catalysis, vide infra); however, harsh conditions $\left(>120^{\circ} \mathrm{C}, 40\right.$ bar) are required, which limit the prospective scope of the transformation 
(Scheme 10a). Because orthogonal relay catalysis enables to select each catalyst for each function independently, the dual-catalytic strategy might facilitate the optimization of the overall activity and selectivity. In that context, Hartwig and co-workers ${ }^{88}$ reported an orthogonal relay protocol for hydroaminomethylation of alkenes, which connects Rh-catalyzed hydroformylation of alkenes $\mathbf{1 0 . 5}$ with in situ Ir-catalyzed reductive amination of aldehyde intermediates $\mathbf{1 0 . 8}$ to form amines 10.7 (Scheme 10b). Although the approach requires two precious metal catalysts, the full sequence occurs under milder conditions $\left(80^{\circ} \mathrm{C}, 3.4\right.$ bar) with superior regioselectivity, and a broader functional group tolerance, when compared with the sequence in the presence of a single catalyst. The comparison of both protocols underscores the advantages of orthogonal relay catalysis. 
Scheme 10. Comparison of mono-catalytic Rh-catalyzed auto-relay protocol (a) and the bicatalytic Rh-/Ir-catalyzed orthogonal relay protocol (b) for direct hydroaminomethylation of terminal alkenes to form amines
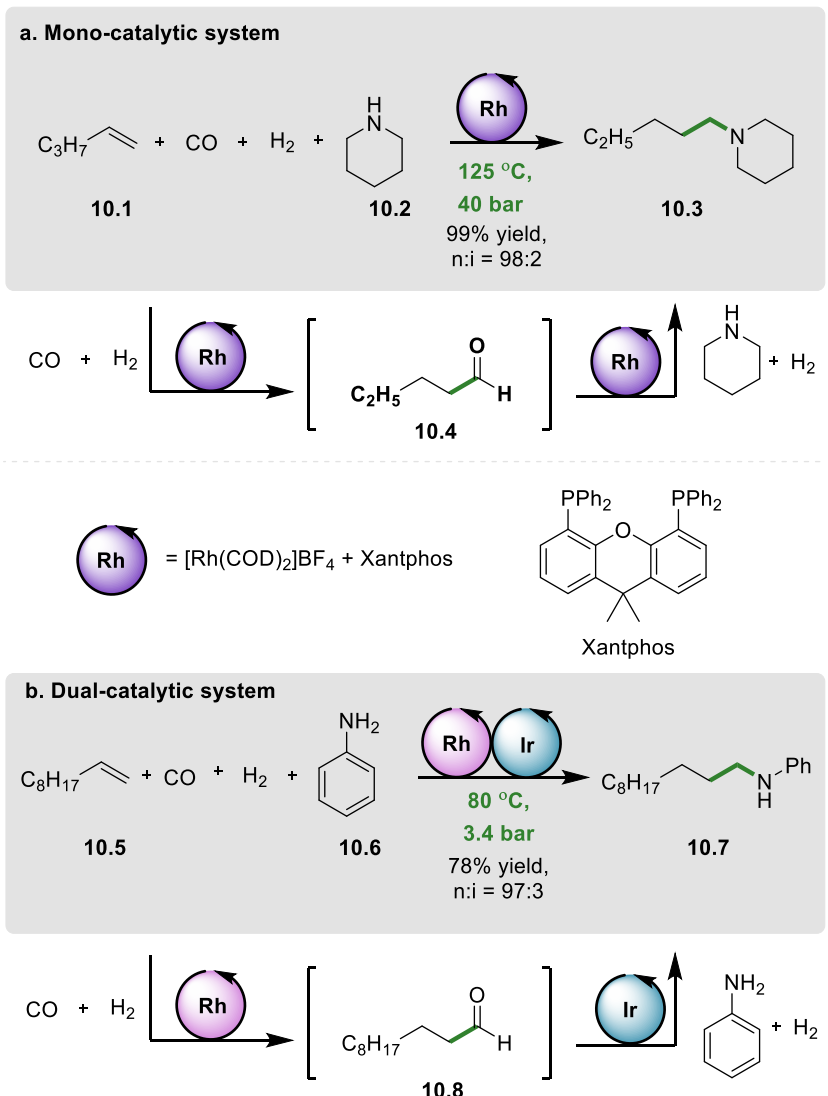

10.8
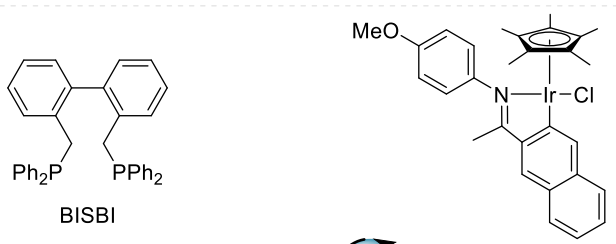

(Rh) $=\left[\mathrm{Rh}(\mathrm{CO})_{2}(\mathrm{acac})\right]+\mathrm{BISBI} \quad \mathrm{Ir}=$ Xiao's catalyst

Key feature:

two catalysts operate two distinct reactions under milder conditions than a single catalyst does, thus increasing the applicability of the method

to a broader range of substrates

\section{$\underline{\text { Including unstable intermediates }}$}

Under orthogonal relay catalysis, an intermediate can be converted further as soon as it is formed, leading to its short residence time in the reaction mixture. Therefore, such pathways of 
reactions can include unstable intermediates, which would be otherwise unfeasible for a stepwise approach. Waldmann and co-workers ${ }^{89}$ developed the enantioselective synthesis of the spirotropanyl oxindole scaffold $\mathbf{1 1 . 3}$ proceeding through the $\mathrm{Rh}$-catalyzed reaction of $(E)$-oximino $\alpha$-diazo ketone $\mathbf{1 1 . 1}$ to form transient azomethine ylide $\mathbf{1 1 . 4}$ that subsequently underwent $\mathrm{Nd}$ catalyzed intermolecular 1,3-dipolar cycloaddition with 3-alkenyl oxindoles 11.2 (Scheme 11a). That one-pot relay approach provides straightforward access to a class of complex biologically active compounds. The synthetic sequence could not be executed in a stepwise manner, due to the instability of the ylide intermediate.

Zhong and co-workers ${ }^{90}$ reported a direct synthesis of benzofuroindolines 11.7. The sequence occurs thorough Mn-catalyzed oxidation of $N$-Boc aminophenols 11.5 to unstable $N$-Boc quinone imines 11.8, which subsequently undergo a phosphoric acid-catalyzed enantioselective 1,3-dipolar cycloaddition with indoles 11.6 (Scheme 8b). The strategy was inspired by related phenol oxidation processes occurring in nature, with the Mn-salen complex mimicking natural metalloenzymes. 
Scheme 11. Orthogonal relay processes occurring through unstable reaction intermediates: (a) Rhcatalyzed formation of labile azomethine ylides followed by Nd-catalyzed enantioselective 1,3dipolar cycloaddition, and (b) Mn-catalyzed oxidation of phenol to labile N-boc quinone imines followed by CPA-catalyzed enantioselective 1,3-dipolar cycloaddition
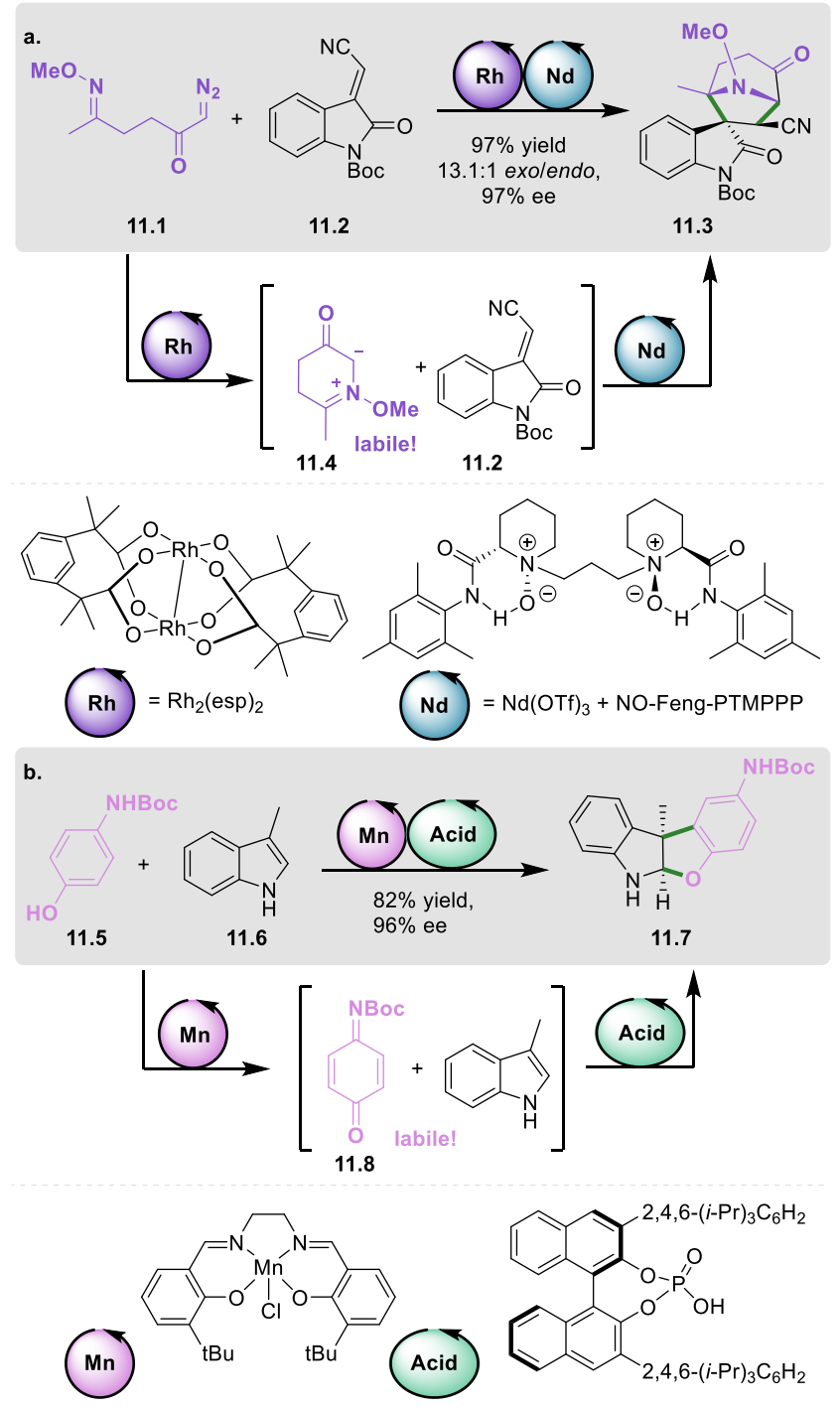

Key feature:

direct conversions of unstable intermediates prevents their decompositions, executing otherwise unfeasible pathways 


\section{Preventing secondary reactions of intermediates}

In many cases, a primary product of a catalytic reaction remains reactive under catalytic conditions, undergoing secondary side reactions that lower the yield of the target transformation. Because under orthogonal relay catalysis, intermediates might be quickly converted in subsequent reactions, such secondary side reactions can be minimized, increasing the yield of the overall transformation. To demonstrate the principle, Hartwig, Zhao, and co-workers ${ }^{91}$ reported a dualcatalytic system that connects a Ru-catalyzed cross-metathesis of (Z)-stilbene $\mathbf{1 2 . 1}$ with 2-butene 12.2 that forms heterocoupling alkene intermediate 12.4 with a subsequent P450-catalyzed substrate-selective epoxidation of intermediate $\mathbf{1 2 . 4}$ to form selectively arylalkyl epoxide $\mathbf{1 2 . 3}$ (Scheme 12). The fast conversion of alkene intermediate $\mathbf{1 2 . 4}$ outcompetes its slow conversion to (E)-stilbene $\mathbf{1 2 . 5}$ in a secondary reaction of the Ru-catalyzed cross-metathesis, leading to higher yields than a corresponding two-step procedure.

Scheme 12. Ru-/P450-catalyzed orthogonal relay of olefin cross-metathesis and substrateselective epoxidation of heterocoupling olefin product

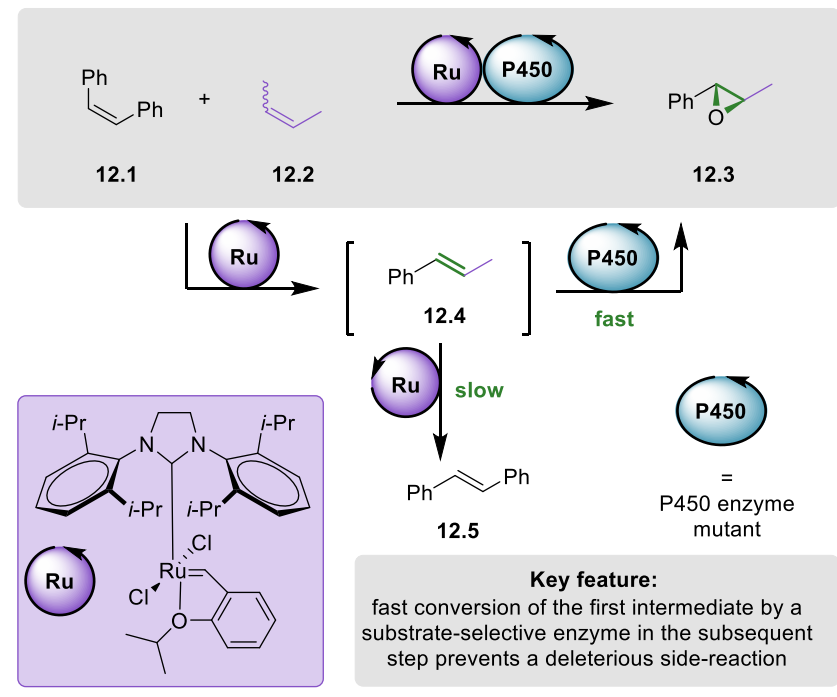




\section{Enabling inaccessible transformations through hydrogen borrowing}

Hydrogen borrowing catalysis ${ }^{92}$ refers to the process exploiting transition metal-catalyzed reversible oxidation of alcohols, amines or alkanes to form transient aldehydes/ketones, imines or alkenes that can undergo a series of new reactions, including typically alkylation or amination, leading directly to value-added compounds. Attractively, the process eliminates any stochiometric redox reactions required otherwise in a stepwise approach. However, by combining the hydrogen borrowing catalysis with catalytic functionalization reactions into dual-catalytic systems, a broad chemical space of new transformations can be envisioned. In that context, Goldman, Brookhart, and co-workers ${ }^{93}$ reported a remarkable dual-catalytic system for the elusive cross-metathesis of linear alkanes (Scheme 13). The sequence involves an Ir-catalyzed reversible dehydrogenation of starting alkane $\mathbf{1 3 . 1}$ to form a transient terminal alkene $\mathbf{1 3 . 3}$, followed by Mo-catalyzed crossmetathesis to yield new larger alkenes $\mathbf{1 3 . 4}$ (and ethylene), which are lastly hydrogenated back to larger alkanes $\mathbf{1 3 . 2}$ by the initial Ir-catalyst. Although, the system suffers from isomerization reactions and harsh reaction conditions, forming a broad distribution of products, the study underscores the unique potential of cooperating reactions under orthogonal dual catalysis.

Following similar design principles, we devised dual-catalytic transition metal systems for functionalization of unreactive sites. ${ }^{94}$ For instance, a relay of reversible oxidation of alcohols and arylation of transient aldehydes enables a general method for direct $\beta$-arylation of alcohols. Specifically, in the system, primary alkyl alcohol $\mathbf{1 4 . 1}$ undergoes reversible oxidation by a Rucatalyst to form transient aldehyde 14.4. The latter undergoes Pd-catalyzed arylation to form aryl aldehyde $\mathbf{1 4 . 5}$ prior to reduction to the $\beta$-aryl alcohol $\mathbf{1 4 . 3}$ by the same hydrogen borrowing Rucatalyst (Scheme 14). In the same vein, the combination of Fe-catalyzed hydrogen borrowing equilibrium with Rh-catalyzed enantioselective hydroarylation of an $\alpha, \beta$-unsaturated aldehydes 
enables a method for direct enantioselective $\gamma$-hydroarylation of allylic alcohols. Importantly, because both reactions building such dual-catalytic systems occur under mild conditions, the overall functionalizations of unreactive sites occur also under mild conditions, enabling the application of such methods to a broad range of starting materials with a high functional group tolerance. Noteworthy, a combination of hydrogen-borrowing catalysis with organocatalysis was reported to enable the control of enantioselectivity of reactions ${ }^{95,96}$ that are otherwise racemic under sole hydrogen-borrowing catalysis. ${ }^{97}$

Scheme13. Ir-/Mo-catalyzed orthogonal relay of alkane dehydrogenation-alkene metathesisalkene hydrogenation for formal alkane cross-metathesis

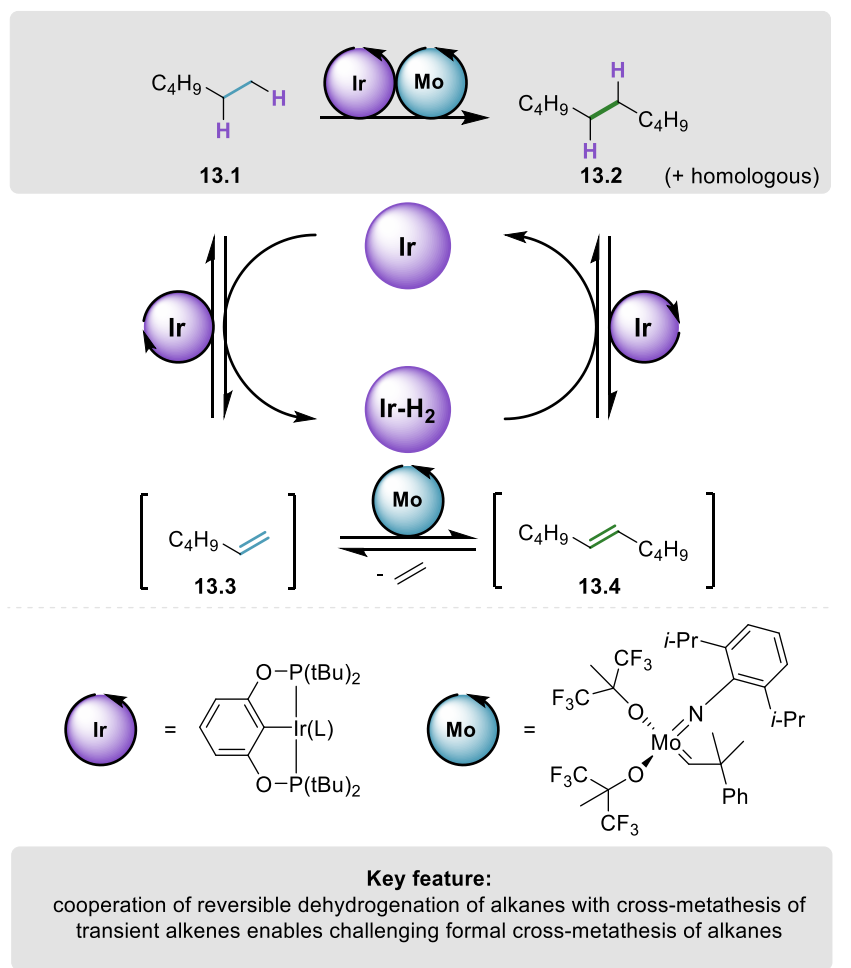


Scheme 14. Ru-/Pd-catalyzed orthogonal relay of alcohol dehydrogenation-aldehyde arylationaldehyde hydrogenation for direct $\beta$-arylation of alcohols

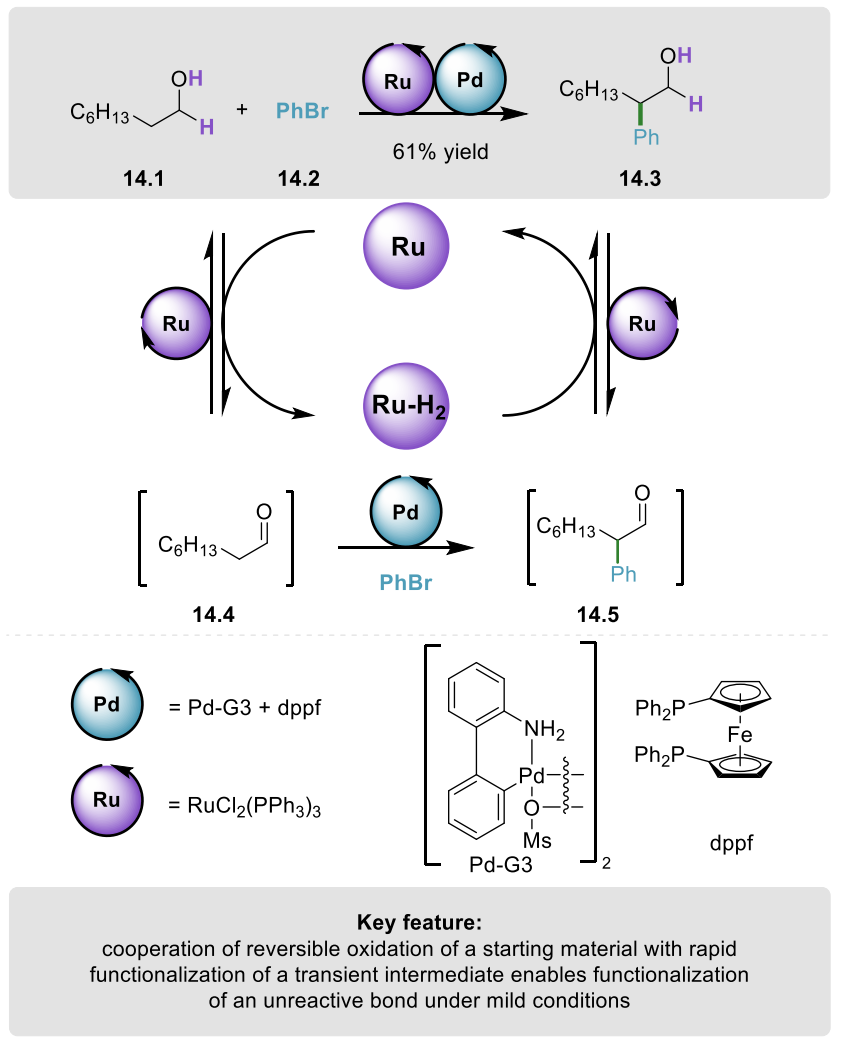

\section{Correcting selectivity of unselective reactions}

Sequences of unselective but reversible reactions cooperating in situ with other selective reactions can increase the selectivity of the overall transformations. For instance, cross-metathesis of two olefins typically leads to the formation of a statistical mixture of a heterocoupling product and undesired homocoupling products, lowering the process efficiency. To address this issue, Hartwig, Zhao, and co-workers ${ }^{98}$ showed that a combination of an unselective but reversible Rucatalyzed cross-metathesis of alkenes $\mathbf{1 5 . 1}$ and $\mathbf{1 5 . 2}$ with a substrate-selective P450-catalyzed epoxidation of olefin 15.4 enables to increase the overall efficiency of the sequence (Scheme 15). Specifically, the selective conversion of heterocoupling alkene $\mathbf{1 5 . 4}$ to epoxide $\mathbf{1 5 . 3}$ drives the reversible metathesis reaction to continuously form a new portion of alkene $\mathbf{1 5 . 4}$ at the expense of 
homocoupling product 15.5 and starting alkene 15.2. The authors showed that in the dual-catalytic system, the overall yield of the target epoxide product $\mathbf{1 5 . 3}$ was 1.5 times higher, while the endconcentration of the self-metathesis product $\mathbf{1 5 . 5}$ was $40 \%$ lower, and the remaining concentration of the starting material $\mathbf{1 5 . 2}$ was $20 \%$ lower, than in the stepwise transformation.

Scheme 15. Ru-/P450-catalyzed orthogonal relay of reversible olefin cross-metathesis and substrate-selective epoxidation of alkenes

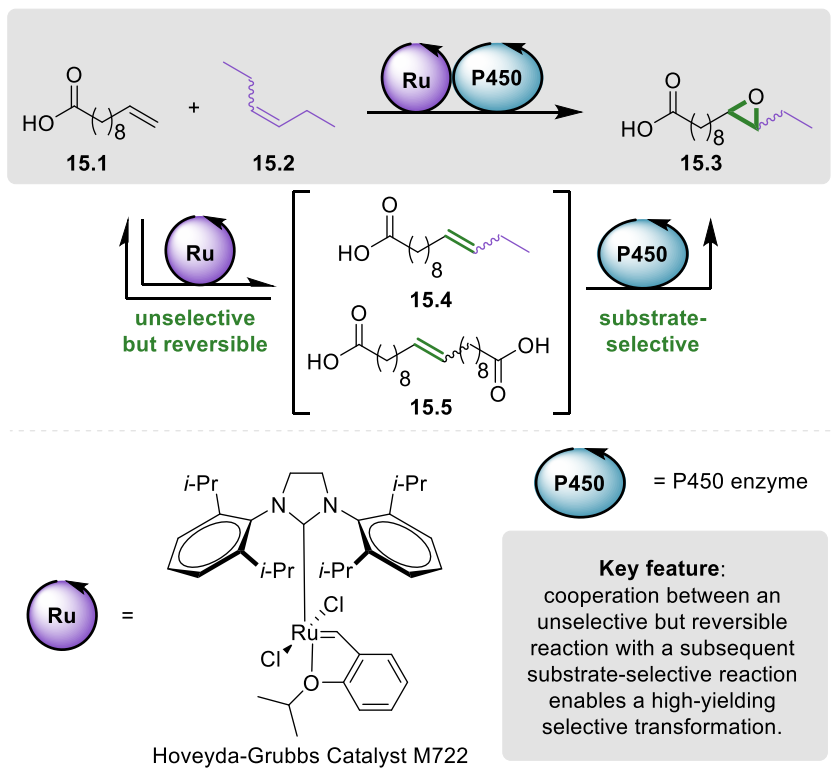

The design principles of the above-described system of cooperating reversible olefin metathesis with selective olefin epoxidation resemble the working principles of dynamic kinetic resolution reactions (DKR) ${ }^{99-102}$ In the latter, a dynamic equilibrium between two enantiomers of a substrate is maintained, but one enantiomer undergoes a subsequent reaction selectively. For instance, a secondary alcohol continues to be reversibly oxidized-reduced to maintain the racemic composition of the mixture in the presence of a hydrogen-borrowing catalyst, but one enantiomer of the alcohol continuous to undergo a selective acylation in the presence of a lipase, leading in the end to a quantitative conversion of a racemic alcohol to an enantiomerically pure ester. This 
strategy has been extensively studied for deracemization of alcohols, amines, aldehydes, and ketones. Recently, a combination of photo- and enzymatic catalysis has been reported, opening up the chemical space of DKR to the elusive stereoselective reduction of mixtures of $(E)$ - and $(Z)$ alkenes. ${ }^{103}$

\section{Exploiting thermodynamic leveraging}

By exploiting the concept of thermodynamic leveraging, orthogonal relay catalysis bears the potential to execute reactions in sequence that are thermodynamically unfeasible. In such case, an endothermic reaction is coupled with a subsequent exothermic reaction to create an overall thermodynamically favorable sequence. For instance, Marks and co-workers ${ }^{104}$ reported a dualcatalytic method for the etheric $\mathrm{C}-\mathrm{O}$ bond hydrogenolysis through a combination of $\operatorname{Ln}(\mathrm{OTf})_{3}-$ catalyzed endothermic reversible C-O bond scission with a subsequent Pd-catalyzed exothermic $\mathrm{C}=\mathrm{C}$ bond hydrogenation to generate an overall favorable process (Scheme 16). Such

transformations can be used to convert biomass-based materials to alkanes, ${ }^{105}$ providing a sustainable alternative to fossil fuels. 
Scheme 16. Thermodynamic leveraging in $\mathrm{Ln}-/ \mathrm{Pd}$-catalyzed orthogonal relay of retrohydroalkoxylation of ethers and alkene hydrogenation drives the etheric $\mathrm{C}-\mathrm{O}$ bond hydrogenolysis

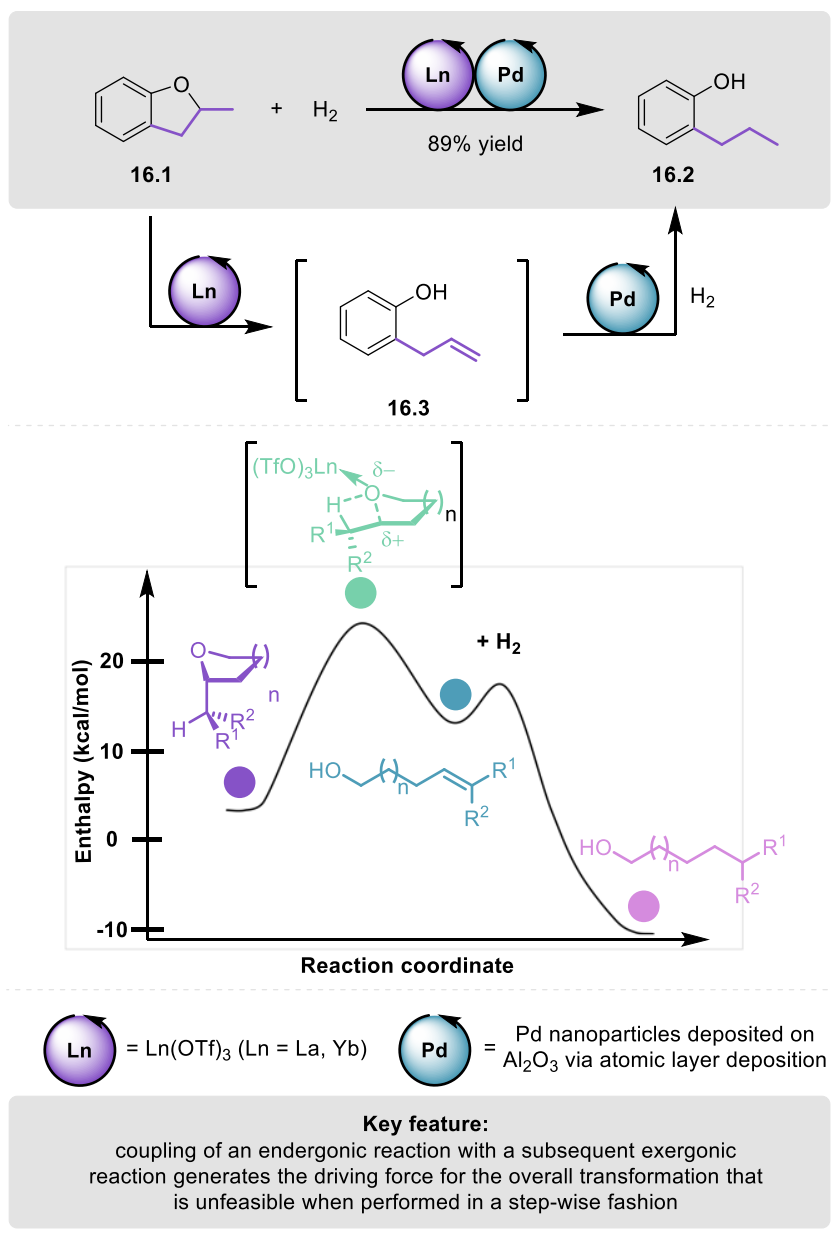

\section{Preventing compatibility issues}

One of the major challenges in the development of orthogonal relay-catalyzed transformations is the prospective incompatibility of catalysts that leads to inhibition of catalytic activity, preventing the overall process to occur. One of the common issues in case of transition metalcatalysis is the prospective ligand scrambling. For instance, in the synthesis of dihydroquinolines 17.3 through a combination of binap-Rh-catalyzed hydroarylation of alkyne $\mathbf{1 7 . 1}$ (with aryl boronic acid 17.2) followed with Xphos-Pd-catalyzed $N$-arylation (Scheme 17), Lautens and co- 
workers ${ }^{106}$ noted that the formation of mixed ligand complexes is detrimental to the catalytic process. However, the ligand exchange between complexes does not occur under the catalytic conditions (or occurs slowly). Therefore, when suitable complexes are formed prior to adding to the reaction mixture, their catalytic activities are maintained, enabling the formation of product 17.3 in $69 \%$ overall yield. Furthermore, this strategy proved effective to prevent premature ligand scrambling in asymmetric variants of orthogonal-relay catalytic reactions in which two metals and two ligands, one chiral and one achiral, are used. ${ }^{107,108}$ Although effective, the approach is not general. Therefore, there have been many other strategies studied to address the incompatibility issues with the physical separation of catalysts. ${ }^{10}$ In such a case, not only prospective ligand exchange processes are hindered but also any cross-reactivity is prevented. For instance, the physical separation of catalysts within the same vessel can be realized by catalysts immobilization on a solid support. ${ }^{109}$ 
Scheme 17. Synthesis of dihydroquinolines in the orthogonal relay of Rh-catalyzed alkyne hydroarylation followed with Pd-catalyzed intramolecular N-arylation
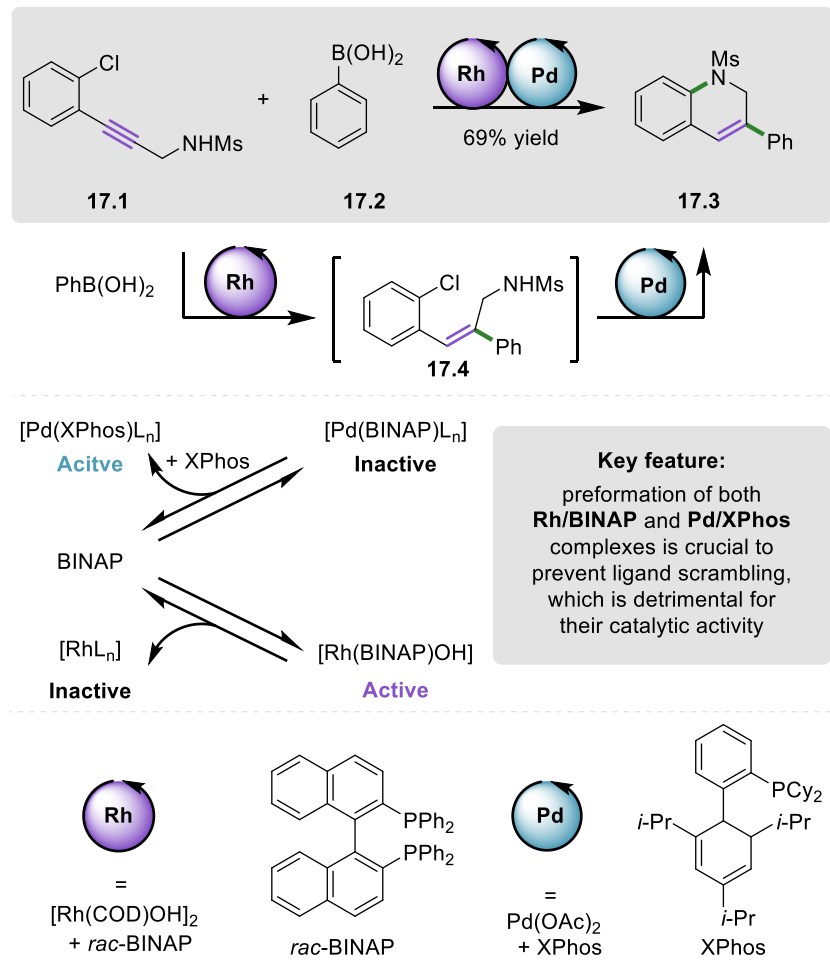

The incompatibility between reagents of one step with the reagents or catalysts of other steps of the sequence might also be encountered in multi-catalytic systems. Strategies to address such incompatibility issues mainly focus on compartmentalization of different reactions, i.e., the catalysts and reagents for different steps are located in different 'compartments' of the reactor. ${ }^{20}$ The compartments can be constructed by phases that are either immiscible, ${ }^{110}$ separated by semipermeable membrane, ${ }^{111}$ or physically separated with the volatile intermediates being exchanged through the gas-phase. ${ }^{12}$ An elegant example of reaction isolation was reported by Weck and coworkers in a sequence of a Co-catalyzed hydration of alkyne $\mathbf{1 8 . 1}$ to form ketone $\mathbf{1 8 . 3}$ and its subsequent Rh-catalyzed enantioselective transfer hydrogenation to form enantioenriched secondary alcohol 18.2 (Scheme 18). ${ }^{113}$ The reactions are not compatible with each other because 
HCOONa, that is, the hydrogen source for the Rh-catalyzed transfer hydrogenation, is not compatible with the Co-catalyst. Therefore, the authors designed a core-shell micellar support constructed in an aqueous environment. The Co-catalyzed hydration takes place in the hydrophobic core, which expels detrimental HCOONa, while the Rh-catalyzed hydrogenation takes place in the hydrophilic shell of the micelle. Notably, the fast diffusion of the ketone intermediate within the core-shell micelle containing both catalysts leads to increased yields, when compared to the control reactions in the presence of a mixture of micelles containing one or the other catalyst. Such spatial organization of catalysts mimics the substrate channeling operating in nature. $^{114}$ 
Scheme 18. Co-/Rh-catalyzed orthogonal relay for the enantioselective conversion of terminal alkynes to secondary alcohols enabled by the compartmentalization of incompatible reactions

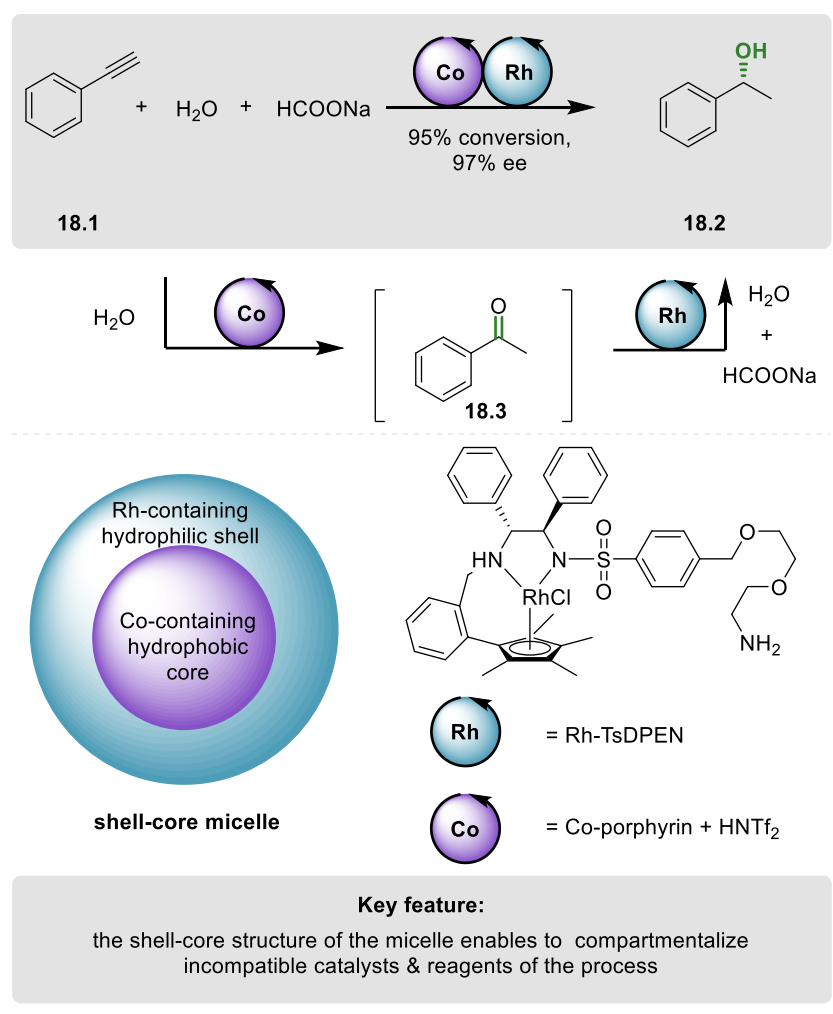

Noteworthy, when biocatalysis and chemocatalysis are both employed, the sequences are typically executed with the aid of multi-phase systems with catalysts operating in different, nonmixable phases, between which the intermediates are exchanged. For instance, the sequence of Ru-catalyzed olefin metathesis and subsequent P450-catalyzed epoxidation (Scheme 15) ${ }^{98}$ was executed in a biphasic system consisting of a dioctylphthalate-phase for the metathetic reaction and an aqueous-phase for the epoxidation reaction. Generally, the strategies for the separation of bio- and chemocatalysts are well-developed, and have been reviewed previously. ${ }^{20}$ Also, the use of artificial biocatalysts, including artificial metalloenzymes, has been reviewed. ${ }^{115,116}$ 


\subsection{Sequential catalysis}

Sequential catalysis refers to catalytic transformations in which the catalysts or reagents for each step are added sequentially. The approach simplifies the way to address the issues of compatibility of catalysts and reagents, optimization of conditions for each step, or synchronization of reactions of the sequence, which are often encountered in orthogonal relay catalysis. However, such an approach prevents exploiting the cooperativity of the reactions, i.e., the enabling feature of relay catalysis that was discussed above. Nevertheless, the main advantage remains in the possibility of performing a target sequence of transformations in one-pot, without the need for costly and tedious isolations and purifications of potentially unstable intermediates.

\section{Preventing side-reactions}

The sequential addition of catalysts helps to eliminate side reactions. For instance, Reek ${ }^{117}$ and co-workers reported a dual-catalytic transition metal system for branch-selective hydroformylation of terminal alkenes, in which two catalysts are added sequentially and the conditions are adapted for each step. The approach prevents secondary reactivity of the first catalyst and eliminates premature reactivity of the second catalyst over the initial substrate. In the first step of the sequence, terminal alkene 19.1 undergoes a Pd-catalyze mono-isomerization to sub-terminal alkene 19.3 (Scheme 19). Upon completion of the first step, a base is added to stop any further isomerization processes, which would lead to a mixture of internal alkenes, i.e., the secondary products of the isomerization reaction. The Rh-catalyst is added, and the reaction mixture is subjected to a syngas atmosphere $\left(\mathrm{CO} / \mathrm{H}_{2}\right)$ to undergo the regioselective hydroformylation of the sub-terminal alkene intermediate 19.3, yielding $\alpha$-methyl-branched aldehyde 19.2 selectively in high yield. 
Scheme 19. Pd-/Rh-catalyzed sequential olefin isomerization - hydroformylation for $\alpha$-branchselective hydroformylation of terminal alkenes

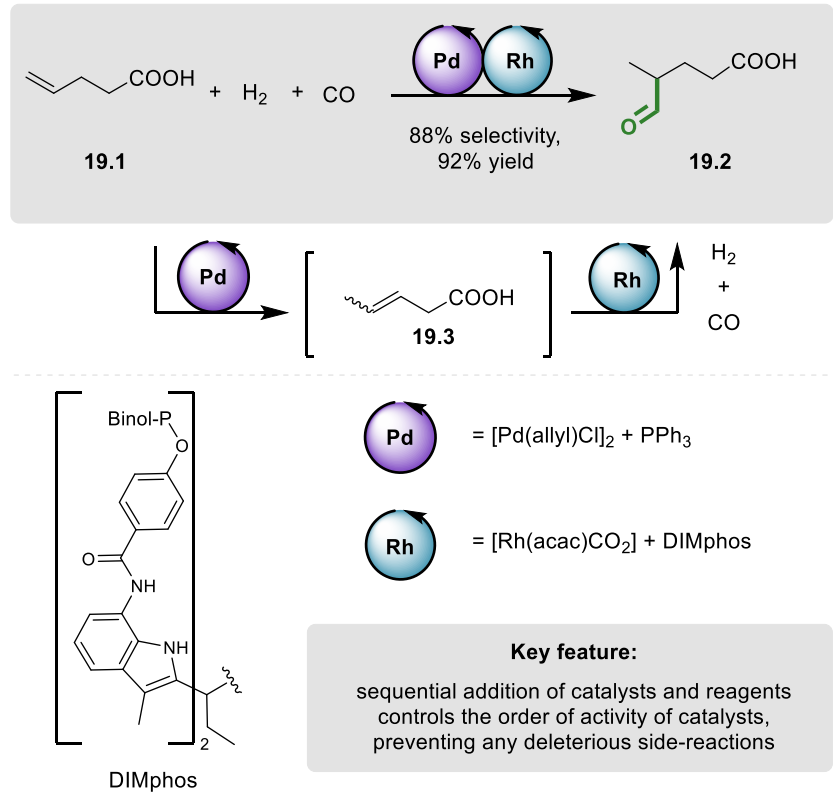

\section{Eliminating incompatibility issues}

The sequential addition of catalysts assists to mitigate the inherent incompatibility between catalysts, such as incompatibility between Brønsted acid and Brønsted base catalysts. For instance, Zhou and co-workers ${ }^{118}$ executed a one-pot three-step enantioselective synthesis of spirocyclic indolines 20.3 employing the acid- and base-catalyzed reactions (Scheme 20). The sequence starts with a Pd-catalyzed hydrogenation of nitroarene $\mathbf{2 0 . 1}$ to form aryl amine 20.4, which is then subjected to a Brønsted acid catalyzed coupling with isatin derivative $\mathbf{2 0 . 2}$ to form ketimine $\mathbf{2 0 . 5}$. Subsequently, a chiral Brønsted base is added to promote the asymmetric $6 \pi$ electrocyclization, yielding chiral product $\mathbf{2 0 . 3}$ in $85 \%$ yield and $92 \%$ ee. Sequential catalysis represents a particular advantage when the stability of intermediates with respect to purification procedures limits the yield of a synthetic route. The authors showed that stepwise synthesis yields the product in only 
$34 \%$ overall yield (with $97 \%$ ee), illustrating both the practicality and the increased efficiency of the one-pot procedure over the classic stepwise approach.

Scheme 20. Pd-/Brønsted acid-/ Brønsted base-catalyzed three step sequence involving reduction, coupling, and asymmetric $6 \pi$ electrocyclization for the formation of spirocyclic indolines

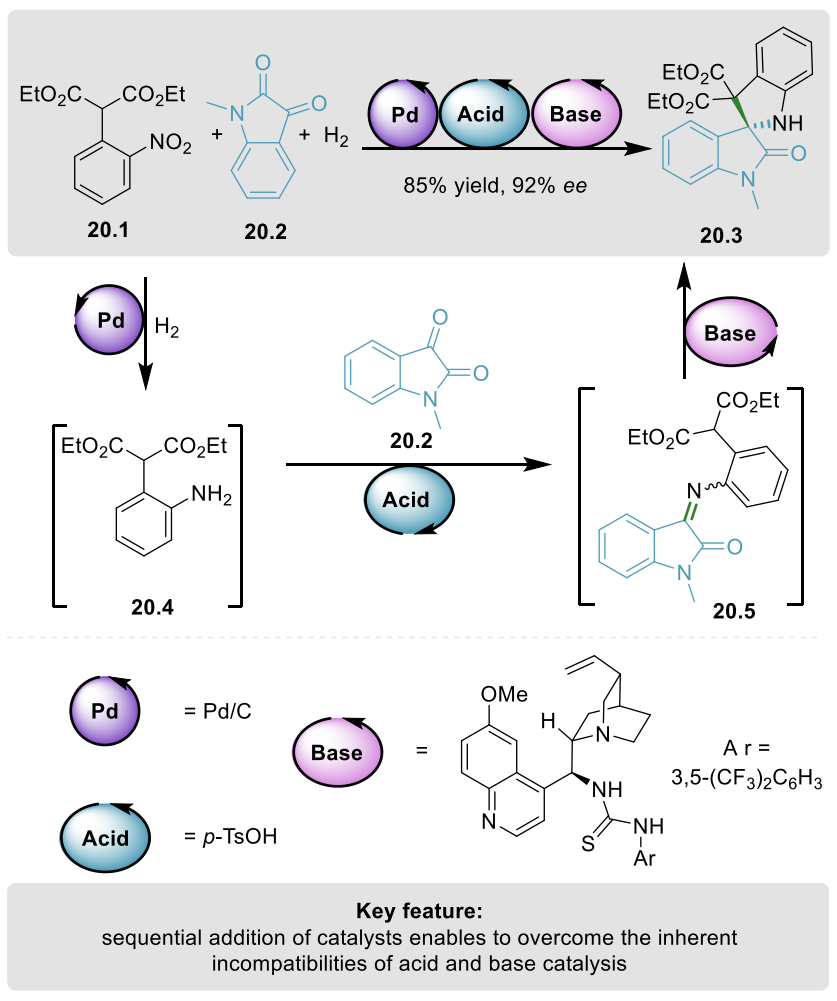

\section{Exploiting multiple transition metal-catalysts}

Although a number of examples of ternary catalytic systems combining transition metal- and organocatalysts operating in a sequential fashion has been reported, ${ }^{2}$ three-step sequences occurring in the presence of three transition metal-catalysts remain rare. The main challenges are driven by both the compatibility requirements between all metal complexes and reagents, and the prospective ligand exchanges between the catalysts that are likely to deteriorate their catalytic activities. However, the potential of such multi-catalytic transformations stimulates the development of the ternary transition-metal systems. Ramasatry and co-workers reported one of 
the first examples of multi-catalytic protocols with three transition metal-catalysts for the 3-step synthesis of $\beta$-carbolines 21.2 (Scheme 21). ${ }^{119}$ Within the process, starting material 21.1 undergoes first Ag-catalyzed intramolecular hydroamination to form allylic alcohol 21.3, which is followed by Bi-catalyzed 1,3-allylic alcohol isomerization (AAI) and the Friedel-Crafts-type dehydrative azidation to afford azide intermediate 21.4. Lastly, the latter undergoes the Pdcatalyzed [3+2]-cycloaddition to form $\mathbf{2 1 . 5}$ followed by nitrogen extrusion to yield $\beta$-carbolines 21.2 in overall $82 \%$ yield. Noteworthy, azide intermediate $\mathbf{2 1 . 4}$ is unstable and potentially explosive. Hence in situ conversion of 21.4, without the need for potentially dangerous isolation, is the key factor for the formation of the final product both in high yield and in a safe manner.

Scheme 21. Ag-/Bi-/Pd-catalyzed three step sequence involving hydroamination, 1,3-allylic alcohol isomerization (AAI), and [3+2] cycloaddition for the formation of $\beta$-carbolines

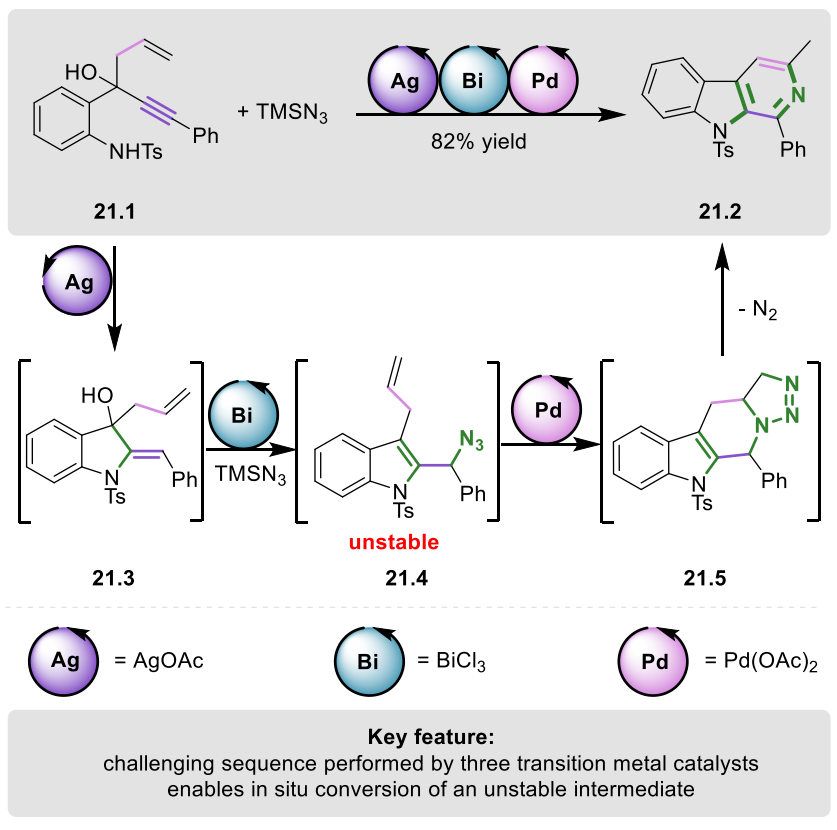




\section{Multiple transition metal-catalysts in stereoselective synthesis}

Compatibility issues aside, the prospective ligand exchange between multiple transition metal catalysts is particularly problematic in case of stereoselective reactions, because any ligand exchange is likely to deteriorate the stereocontrol of the overall transformation. However, because the efficient preparation of chiral molecules is the key in fine-chemical synthesis, stereoselective multi-catalytic systems remain of high interest. We have recently developed multi-catalytic transition-metal protocols that convert alkenes, unsaturated aliphatic alcohols, and aryl boronic acids to form secondary benzylic alcohols, a prevalent moiety in biologically active molecules and valuable building blocks in organic synthesis, with high stereoselectivities, within one-pot sequences of reactions by integrating transition-metal catalyzed alkene cross-metathesis, isomerization, and nucleophilic addition reactions (Scheme 22a). ${ }^{120}$ Because each reaction of the sequence is executed by an independent catalyst, allylic alcohols bearing a prochiral double bond 22.8 can be converted to any stereoisomer of the product $\mathbf{2 2 . 9}$ with high stereoselectivity ( $>98: 2$ er and >20:1 dr, for 1,3-syn and 1,3-anti selective synthesis; Scheme 22b). Overall, with the aid of up to four catalysts operating in a single vessel, the protocols directly convert simple starting materials into a range of valuable products with high stereocontrol. Not only the one-pot protocols are operationally simpler and up to $\sim 3$-fold resources more efficient than the stepwise syntheses, but also the overall yield of the product is increased thanks to preventing cumulative losses of materials during subsequent isolations and purifications of the intermediates. Overall, the approach simplifies the synthesis of target motives, increases material efficiency, and limits cost, time, and waste associated with the multi-step procedures. 
Scheme 22. Multi-catalytic sequences for stereoselective synthesis of secondary benzylic alcohols with up to four catalysts working in the same reaction mixture

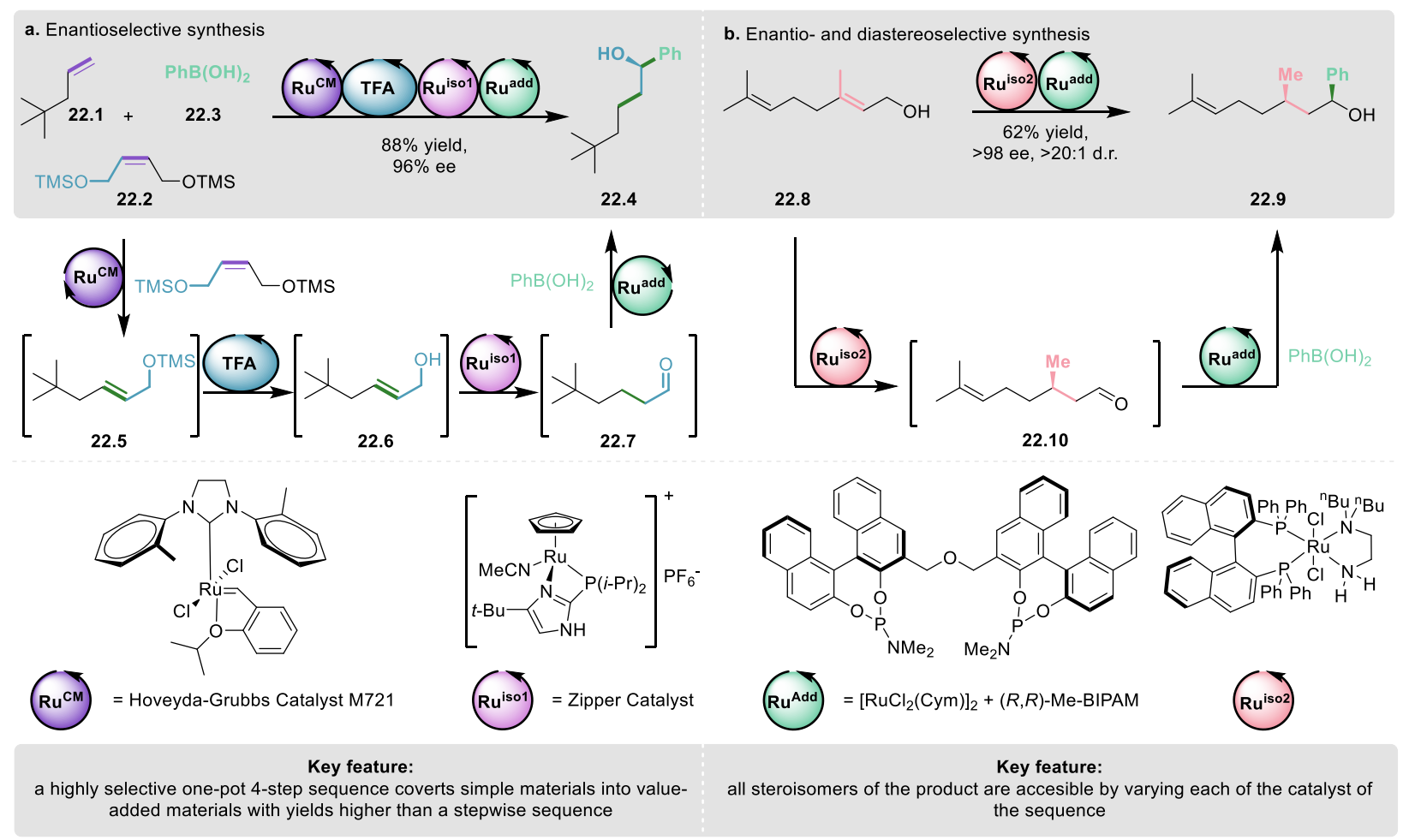

\subsection{Auto-relay catalysis}

Auto-relay catalysis, referred to as auto-tandem catalysis when two transformations take place, refers to catalytic systems in which a single (pre)-catalyst catalyzes multiple, independent reactions within mechanistically distinct catalytic cycles under the same reaction conditions with all reagents present from the start. The approach faces different challenges than orthogonal relay catalysis. Because a single catalyst is used, incompatibility issues concerning the catalysts are obsolete. The main challenge relates to designing a pre-catalyst that can catalyze two reactions of different catalytic mechanisms in a well-defined manner under the same conditions. The rates of each step are interrelated, and the starting materials and intermediates need to be reactive only in a specific 
step. Because catalytic activities are promoted by the same pre-catalyst, optimization of each step is challenging with a change of a single parameter affecting all reactions within the sequence. The field has been a subject of a micro-review ${ }^{121}$ and conceptual article. ${ }^{122}$ Selected examples are discussed below.

The study by Onitsuka and co-workers ${ }^{123}$ for the synthesis of enantiomerically enriched $\gamma$ lactams $\mathbf{2 3 . 3}$ constitutes an elegant example illustrating the development of auto-relay catalytic systems. The authors envisioned a sequence connecting previously established asymmetric allylic substitution $(\mathrm{AAS})^{124}$ of chloroalkene 23.1 with N-nucleophile $\mathbf{2 3 . 2}$ to form bromoalkene intermediate 23.4 with its subsequent atom transfer radical cyclization (ATRC) to yield the final product 23.3 (Scheme 23). Both reactions are catalyzed exclusively by a cyclopentadienylruthenium (Cp'Ru) complex through two different mechanisms. While the AAS reaction occurs through the 2-electron $\mathrm{Ru} / \mathrm{Ru}^{\mathrm{IV}}$ mechanism, the ATRC cyclization reaction occurs through the radical $\mathrm{Ru}^{\mathrm{II}} / \mathrm{Ru}^{\mathrm{III}}$ mechanism. Overall, the protocol directly yields the target $\gamma$-lactams 23.3 in excellent yields and enantioselectivities. 
Scheme 23. Ru-catalyzed auto-relay of asymmetric allylic substitution (AAS) and atom transfer radical cyclization (ATRC) for enantioselective synthesis of $\gamma$-lactams

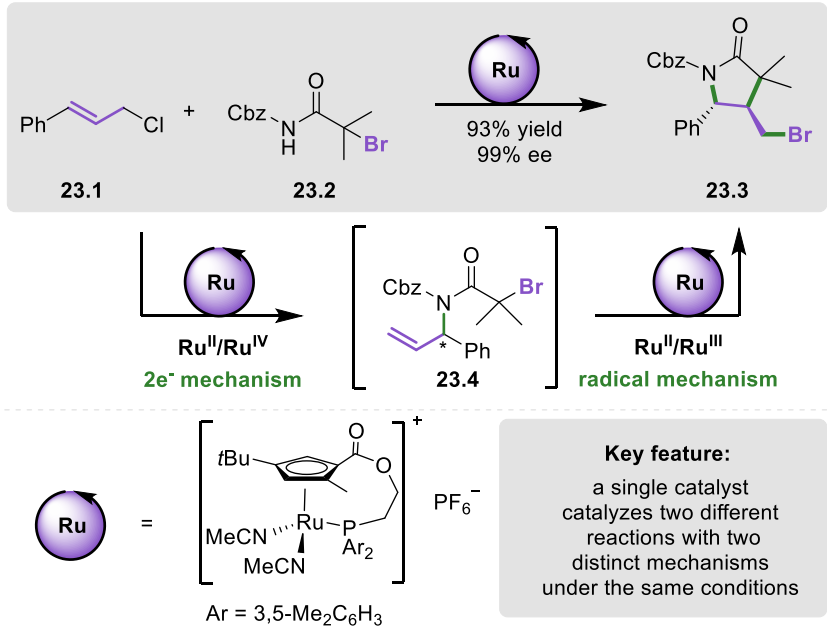

The field of auto-relay catalysis is dominated by transition metal-based catalytic systems, including recent elegant studies on Ni-catalyzed direct transformations of dienyl allylic alcohols into chiral 1,3-dinitriles by Fang ${ }^{125}$ and Rh-catalyzed dehomologation of alcohols into alkenes by Dong $^{126}$; however, organocatalytic auto-relay catalytic systems have been developed as well. Radosevich ${ }^{127}$ and co-workers reported a system based on a single redox-active organophosphorus catalyst, which operates two complementary cycles in the $\mathrm{P}^{\mathrm{III}} / \mathrm{P}^{\mathrm{V}}$ couple, to execute a condensation-cyclodehydration sequence for amines $\mathbf{2 4 . 1}$ and carboxylic acids $\mathbf{2 4 . 2}$ as starting materials (Scheme 24). The key common intermediate, bromophosponium cation 24.5, is generated upon reduction of phosphine oxide pre-catalyst $\mathbf{R}_{\mathbf{3}} \mathbf{P}=\mathbf{O}$ by phenylsilane and subsequent halophilic reaction with the oxidant DEBM (diethyl bromomalonate). Condensation involves the activation of acid $\mathbf{2 4 . 2}$ with cation $\mathbf{2 4 . 5}$ to form activated onium intermediated 24.6, which in turn reacts with amine $\mathbf{2 4 . 1}$ to form amide intermediate $\mathbf{2 4 . 4}$ within the $\mathrm{C}-\mathrm{N}$ bond forming cycle. Then, amide $\mathbf{2 4 . 4}$ reacts again with catalytic cation $\mathbf{2 4 . 5}$ to form the final product $\mathbf{2 4 . 3}$ through activated onium intermediate $\mathbf{2 4 . 7}$ within the $\mathrm{C}-\mathrm{C}$ bond forming cycle. 
Scheme 24. Condensation-cyclodehydration sequence occurring in the presence of a single organocatalyst that operates two complementary and functionally distinct catalytic cycles
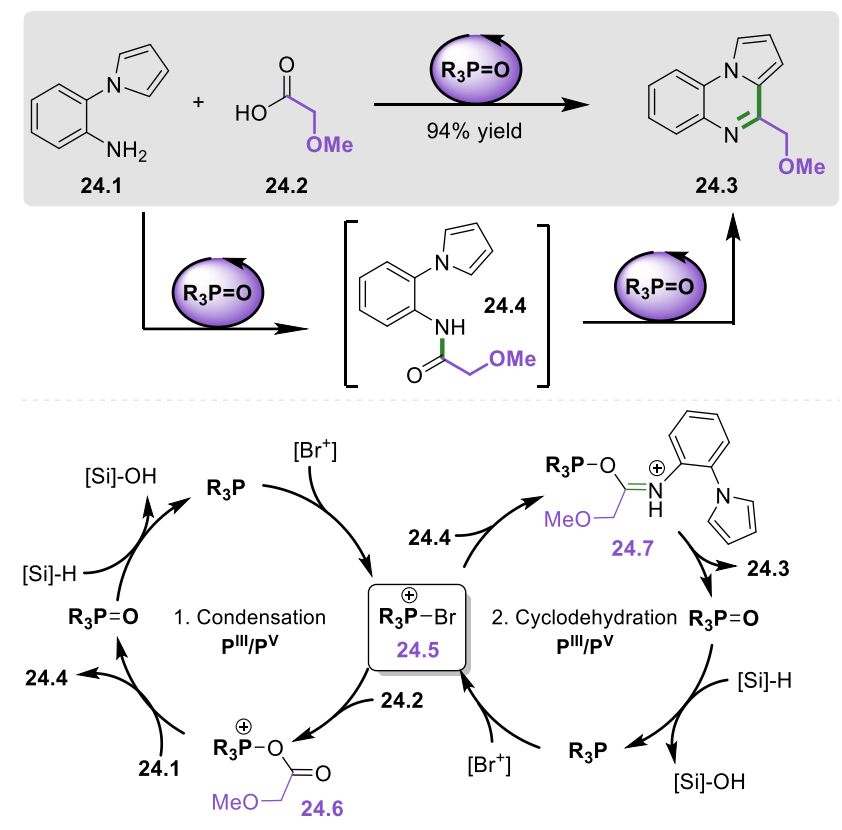

$\left[\mathrm{Br}^{+}\right]=\mathrm{DEBM}$ (diethyl bromomalonate); [Si]-H = $\mathrm{PhSiH}_{3}$

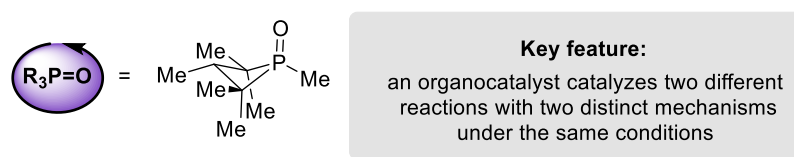

Reaction ordering by 'delay loop'

A major challenge in auto-relay catalysis is to establish kinetic hierarchy for each catalytic cycle involved when the intrinsic reactivity order does not match the required sequence and leads to detrimental side reactions. With the aim to provide an easy access to enantioenriched unprotected homoallylic amines, a valuable building block in organic synthesis, Hoveyda ${ }^{128}$ and co-workers studied a single copper-based catalytic system to promote reductive coupling of allenes and nitriles (Scheme 25). Two functionally different catalytic cycles operate simultaneously to catalyze allene 25.1 addition to a nitrile 25.2, enabled by $\mathrm{Cu}-\mathrm{B}$ (pin) species, and ketimine $\mathbf{2 5 . 5}$ reduction enabled by $\mathrm{Cu}-\mathrm{H}$ species. However, premature addition of $\mathrm{Cu}-\mathrm{H}$ to allene lead to side-products, lowering 
the efficiency of the overall process. To address this issue, the authors introduced a non-productive 'delay loop' by adding a sacrificial reagent, which reacts with $\mathrm{Cu}-\mathrm{H}$ faster than the allene does, and hence holds the reduction step just until the ketimine intermediate is formed; the latter reacting with $\mathrm{Cu}-\mathrm{H}$ faster than the sacrificial reagent. Overall, the delay loop ensures the required order of the catalytic events is kept, albeit at the price of non-productive consumption of sacrificial reagents.

Scheme 25. Cu-catalyzed auto-relay of reductive coupling of allenes and nitriles for the preparation of $\mathrm{NH}_{2}$-amines

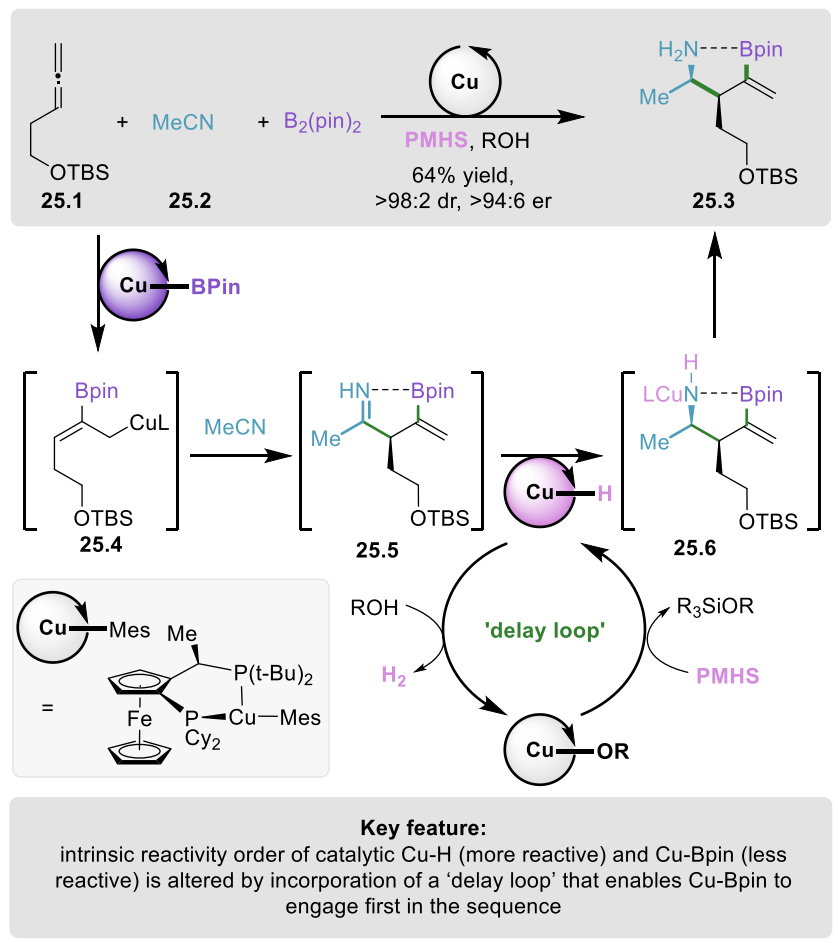




\section{$\underline{\text { Pseudo-allosteric regulation of catalytic activity }}$}

Another strategy to ensure precise ordering of catalytic activities is to separate the operation of different catalytic cycles in the time domain through a pseudo-allosteric control. Herzon ${ }^{129}$ and coworkers reported an example in which two catalytic functions of a Ru-complex - hydration and reduction - are separated in time (Scheme 26). The key aspect of this system is that the resting states of the catalyst in each catalytic cycle are metal-substrate complexes. Therefore, the substrate that binds the strongest to the Ru-resting state dominates its catalytic activity until this substrate is consumed. Within the sequence, first a strongly binding alkyne 26.1 undergoes anti-Markovnikov hydration to the corresponding aldehyde 26.4, which in turn undergoes uncatalyzed Wittig olefination to form 1,4-enone intermediate 26.6. Because the reduction activity of the catalyst remains latent in the presence of alkyne 26.1, the side-reduction of the aldehyde 26.4 to the alcohol 26.5 is inhibited, so the intermediate is selectively converted to 1,4-enone $\mathbf{2 6 . 6}$. When conversion of alkyne $\mathbf{2 6 . 1}$ is finished, 1,4-enone $\mathbf{2 6 . 6}$ can bind to the catalyst to be reduced to the ketone $\mathbf{2 6 . 3}$ through transfer-hydrogenation, forming the product in high yield (up to 75\%). The overall control of activity resembles the allosteric mechanism controlling activity of enzymes, that is, one of the key features enabling biosynthetic pathways to be executed through well-orchestrated enzymatic reactions. 
Scheme 26. Ru-catalyzed auto-relay of alkyne hydration, Wittig olefination, and transferhydrogenation with a pseudo-allosteric regulation of catalytic activity

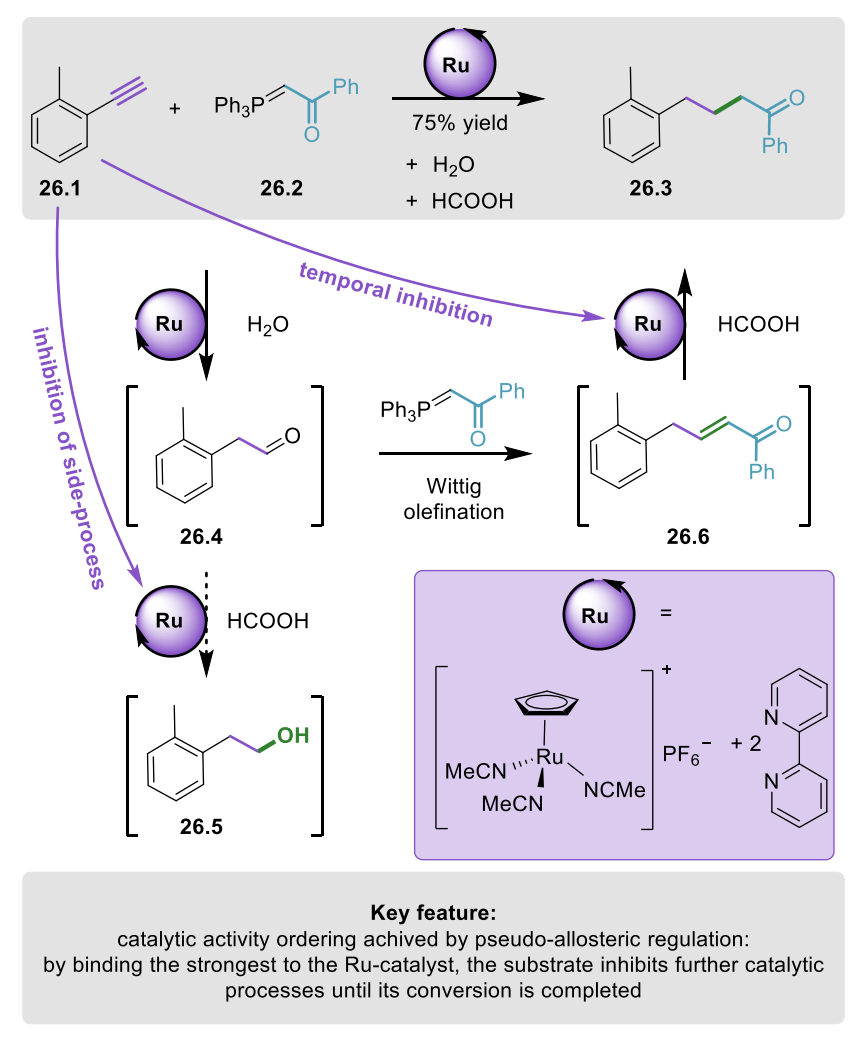

\subsection{Assisted-relay catalysis}

Assisted-relay catalysis refers to catalytic systems in which a single catalyst operates multiple reactions within different catalytic cycles, but the subsequent reactions are triggered by the addition of reagents or change in the reaction conditions. Because optimal conditions for each step can be easily adjusted, the order of steps can be controlled. However, because of the time separation of the different catalytic cycles, cooperation between reactions remains excluded.

\section{Temperature trigger}

Temperature changes are often used to change the activity of the catalyst. Recently, Ackermann ${ }^{130}$ and co-workers reported sequential meta-C $-\mathrm{H}$ alkylation and ortho- $\mathrm{C}-\mathrm{H}$ arylation of aryloxazoline $\mathbf{2 7 . 1}$ occurring in the presence of a single ruthenium(II) complex (Scheme 27). 
First, the starting material undergoes meta-selective C-H-alkylation with alkyl bromide $\mathbf{2 7 . 2}$ through the radical $\mathrm{Ru}^{\mathrm{II}} / \mathrm{Ru}^{\mathrm{III}}$ mechanism. After completion of the first step, aryl bromide $\mathbf{2 7 . 3}$ is added, and the reaction temperature is increased to trigger Ru-catalyzed ortho-selective C-Harylation through the two-electron $\mathrm{Ru}^{\mathrm{II}} / \mathrm{Ru}^{\mathrm{IV}}$ mechanism to form the double $\mathrm{C}-\mathrm{H}$ functionalized product $\mathbf{2 7 . 4}$ in one-pot in $73 \%$ overall yields.

Scheme27. Ru-catalyzed temperature-controlled assisted-relay of sequential C-H alkylation via radical mechanism and ortho- $\mathrm{C}-\mathrm{H}$ arylation via $\mathrm{Ru}{ }^{\mathrm{I} /} / \mathrm{Ru}^{\mathrm{IV}}$ mechanism

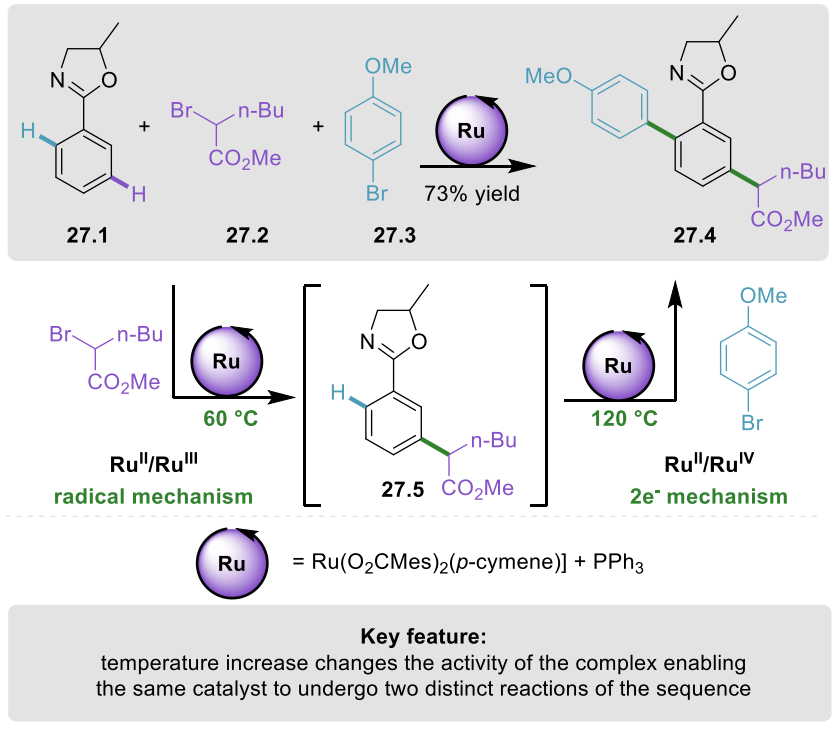

\section{Chemical trigger}

Chemical triggers can be used to trigger the activity change of a catalyst in assisted-relay catalysis. Choudhury ${ }^{131}$ and co-workers reported a $\mathrm{Ru}(\mathrm{II})$-complex, which is able to adopt two different states for different catalytic functions: the first state for dehydrogenative coupling of amines, and the second state for hydrogenation of imines (Scheme 28). Both states of the catalyst are switched by the addition of an acid or a base. In a model experiment, 4-methylbenzylamine 28.1 undergoes dehydrogenative coupling to form the corresponding imine intermediate $\mathbf{2 8 . 3}$ in the presence of the catalyst in the first state. Upon addition of a base, the Ru-catalyst adapts the 
second state, which undergoes hydrogenation of the imine intermediate $\mathbf{2 8 . 3}$ in the presence of hydrogen gas, furnishing the dibenzylamine product $\mathbf{2 8 . 2}$ in $70 \%$ yield.

Scheme 28. Ru-catalyzed pH-controlled assisted-relay of sequential dehydrogenative coupling of amines followed with hydrogenation of the imine intermediate

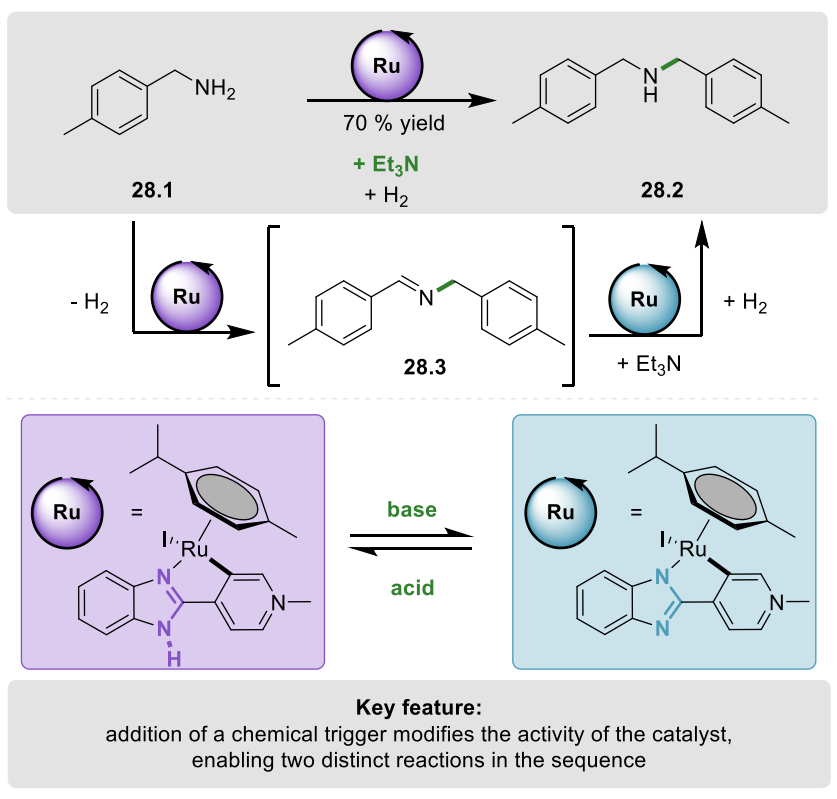

\section{Complex systems}

While most multi-catalytic systems involve just two reactions or two catalysts and can be assigned to a single class of cooperative, domino, or relay catalysis, a higher level of complexity becomes increasingly more common. Although the development of such systems is particularly challenging, due to increasing demand for the precise ordering of the catalytic events and preventing any incompatibility issues or side-reactions between multiple catalysts and reagents, enormous potential for achieving highly efficient synthetic transformations motivates the research in the field. Within this chapter, we discuss selected examples that illustrate various combinations of cooperative, domino, and different types of relay catalysis. The selected studies highlight both increasing complexity and synthetic potential of such multi-catalytic approaches to access 
increasingly complex architectures from simple starting materials within sequences of precisely orchestrated catalytic events.

We classified the studies of complex systems into two main categories: (a) unassisted complex systems, in which all reactions of the sequence occur without any change to the conditions or any additions of reagents or catalysts, and (b) assisted complex systems, in which the sequence requires the conditions to be modified or reagents/catalysts to be added in the course of the process to trigger subsequent transformations.

\subsection{Unassisted complex systems:}

Unassisted complex systems that contain all reagents and catalysts from the start are particularly challenging to design, due to the requirement of compatibility between multiple reagents and catalysts, precise ordering of the catalytic events, and preventing any side-reactions. However, such systems offer the broadest realm of opportunities by merging the advantages of cooperativity between catalysts (i.e., cooperative catalysis) and cooperativity between reactions (i.e., relay catalysis). Below we discuss selected examples of complex systems exploiting either orthogonalor auto-relay catalysis together with cooperative catalysis.

Orthogonal relay catalysis with the $2^{\text {nd }}$ step involving cooperative catalysis (4 catalysts):

Gong $^{132}$ and co-workers reported a protocol exploiting four catalysts to combine hydroformylation of alkenes and asymmetric allylation of aldehydes. The sequence directly converts styrene derivatives, allylic alcohols, and syngas (i.e., $\mathrm{CO}$ and $\mathrm{H}_{2}$ ) to form $\alpha$-quaternary aldehydes enantioselectively (Scheme 29). First, styrene $\mathbf{2 9 . 1}$ undergoes hydroformylation with $\mathrm{H}_{2}$ and $\mathrm{CO}$ to form $\alpha$-branched aldehyde $\mathbf{2 9 . 4}$ in the presence of a Rh-catalyst. In the meantime, the reaction of allylic alcohol 29.2, a $\operatorname{Pd}(0)$-complex, and a chiral phosphoric acid forms a chiral $\pi$-allyl Pd species. The aldehyde intermediate $\mathbf{2 9 . 4}$ reacts with an amine catalyst to form an 
enamine species 29.5, which undergoes asymmetric allylation with the $\pi$-allyl Pd species, exploiting triple-catalytic cooperative catalysis to form quaternary imine enantioselectively. Upon imine hydrolysis $\mathbf{2 9 . 6}$, the final $\alpha$-quaternary chiral aldehyde 29.3 is released. Overall, two C-C bonds are created to form all-carbon quaternary center in a stereoselective fashion with the aid of four catalysts.

Scheme 29. Rh-/Pd-/Brønsted acid-/amine-catalyzed relay involving hydroformylation and cooperative asymmetric allylation to convert vinyl arenes, allylic alcohols, and syngas to form $\alpha$ quaternary chiral aldehydes

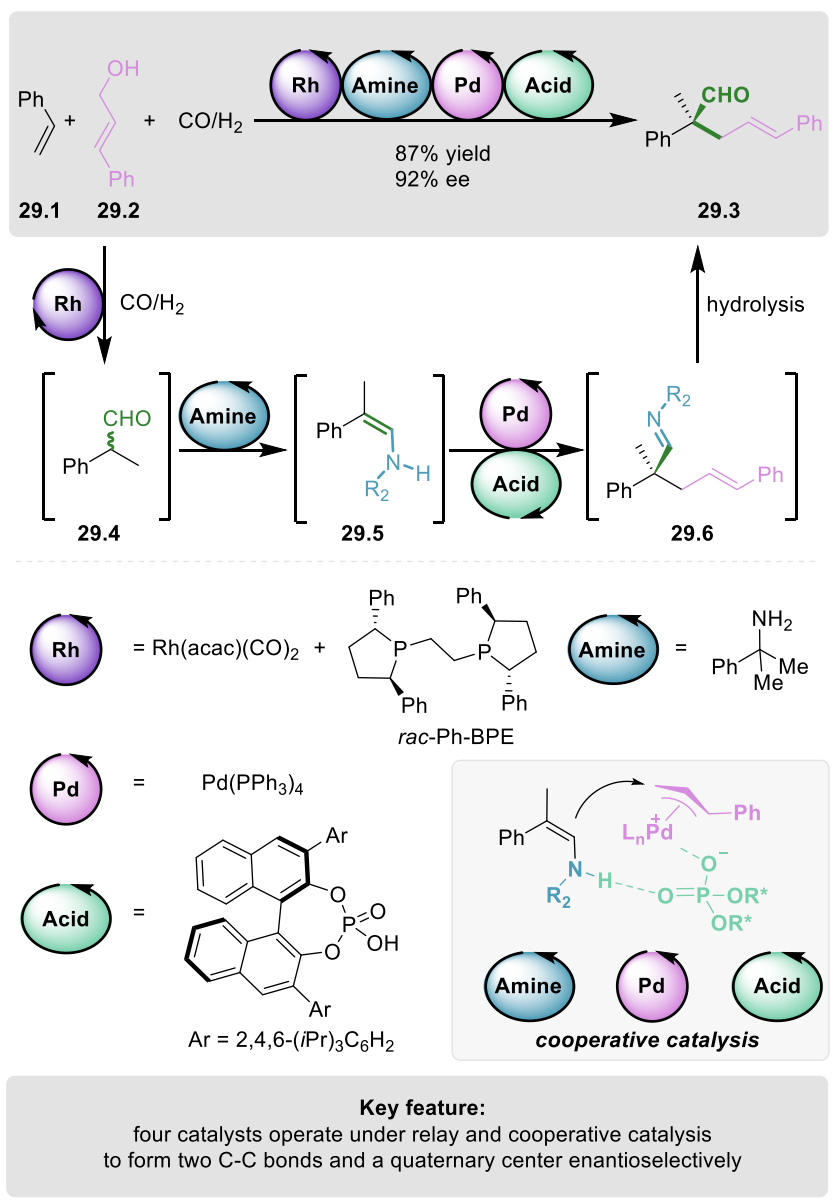

Gong ${ }^{133}$ and co-workers reported a sequence of hydroamination and allylic alkylation reactions occurring in the presence of three catalysts operating under orthogonal relay- and cooperative 
catalysis (Scheme 30). The sequence starts with an Au-catalyst triggering intramolecular hydroamination of secondary amine-bridged enyne $\mathbf{3 0 . 1}$ to form $\mathrm{N}$-allylic enamine intermediate 30.3. Then, the $\mathrm{N}$-allylic enamine undergoes intramolecular allylic alkylation under cooperative catalysis of a Pd-complex and a Brønsted acid to yield final pyrrolidine derivative $\mathbf{3 0 . 2}$.

Scheme 30. Au-/Pd-/Brønsted acid-catalyzed relay of intramolecular hydroamination and cooperative allylic alkylation for transforming secondary amine-bridged enynes into pyrrolidine derivatives

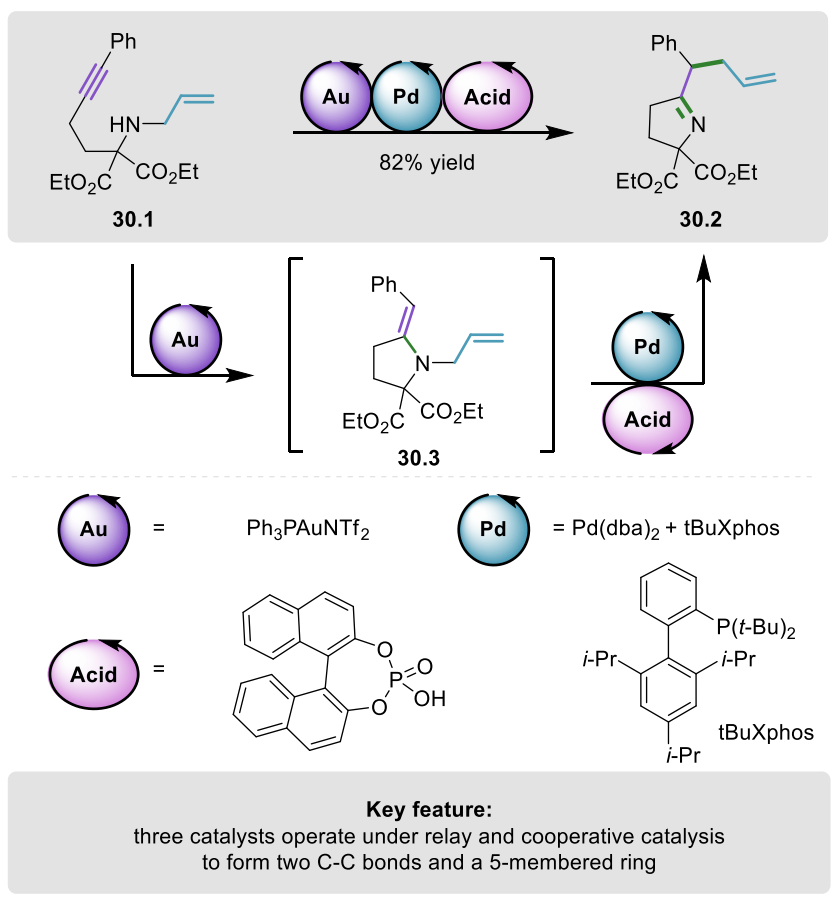

Auto-relay catalysis with the $2^{\text {nd }}$ step involving cooperative catalysis (one catalyst acts both cooperatively and non-cooperatively):

Gong $^{134}$ and co-workers reported enantioselective one-pot synthesis of julolidine derivatives, exploiting two catalysts that operate under auto-relay and cooperative catalysis (Scheme 31 ). The synthesis embarks with phosphoric acid-catalyzed enantioselective [4+2] cycloaddition of the aryl imine formed in situ (from 31.1 +31.2) and enamine 31.3 to form tetrahydroquinoline intermediate 
31.5 bearing an alkyne moiety. Subsequently, the latter undergoes intramolecular hydroamination to form dehydrojulolidine product $\mathbf{3 1 . 6}$ in the presence of cooperative gold phosphate and phosphoric acid catalysts. The synthesis is completed by the stochiometric reduction with a borohydride reagent to form julolidine derivative $\mathbf{3 1 . 4}$ in overall high yield and enantioselectivity. Scheme 31. Brønsted acid-/Au-catalyzed auto-relay of enantioselective [4+2] cycloaddition and cooperative intramolecular hydroamination for the synthesis of julolidine derivatives

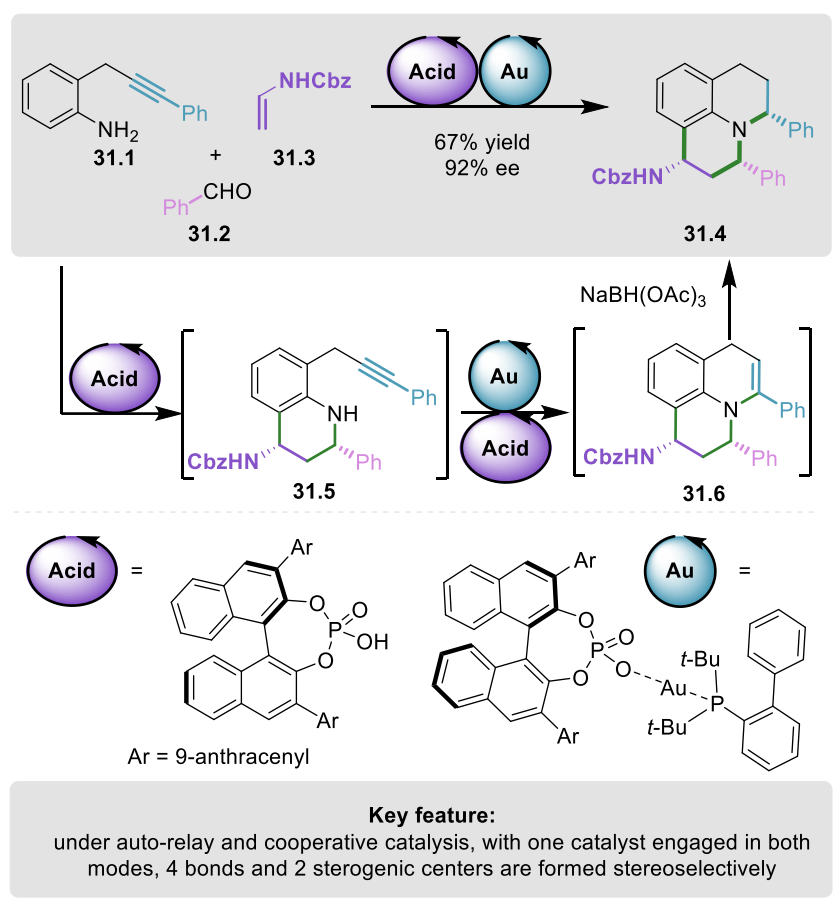

Cooperative and orthogonal relay catalysis (two independent cooperative systems, consisting of three and two catalysts, respectively, work in relay,):

Merging racemic photoredox/organo dual-catalytic reactions ${ }^{135}$ with enzymatic kinetic resolution can serve as a platform for executing elusive stereoselective functionalization reactions at remote, unactivated sites of starting materials. In that context, MacMillan, Hyster, and coworkers ${ }^{136}$ recently reported an approach in which a racemic mixture of $\beta$-substituted ketone, $(S)$ 32.1 and $(R)$-32.2, is converted to nearly enantiopure $\gamma$-substituted alcohol $\mathbf{3 2 . 3}$ by merging a 
substrate-selective reduction process operated by two enzymes with a racemization sequence enabled by three cooperative catalysts (Scheme 32). In the system, $(R)-\mathbf{3 2 . 2}$ is reduced stereoselectively to alcohol $\mathbf{3 2 . 3}$ by substrate-selective NADPH-dependent ketoreductase working in combination with glucose dehydrogenase. Importantly, $(S)$-32.1 remains unreactive to the enzyme. However, $(S)$-32.1 undergoes continuous racemization in the system with the aid of three cooperating catalysts under light irradiation. First, $(S)$-32.1 undergoes condensation with amine 32.4 to form enamine $(S)-32.5$, which is oxidized to enaminyl radical cation $(S)-32.6$ by the excited iridium photocatalyst. Upon subsequent deprotonation of $(S)-\mathbf{3 2 . 6}$ at its allylic site, the stereochemical information is lost and prochiral radical intermediate 32.7 is formed. An unselective HAT between 32.7 and the thiol catalyst forms a racemic mixture of $(S)$-32.5 and $(R)$ 32.8. Upon hydrolysis, $(R)-32.2$ is formed, which undergoes selective reduction to product $\mathbf{3 2 . 3}$, and (S)-32.1, which enters again the racemization cycle. This unique protocol that exploits five catalysts operating in parallel demonstrates the feasibility of catalytic systems of increasing complexity to execute attractive transformations that remain beyond the capabilities of standard systems. 
Scheme 32. Merging photocatalysis with enzymatic reactions enables stereoselective reactions at traditionally static stereocentres of racemic starting materials

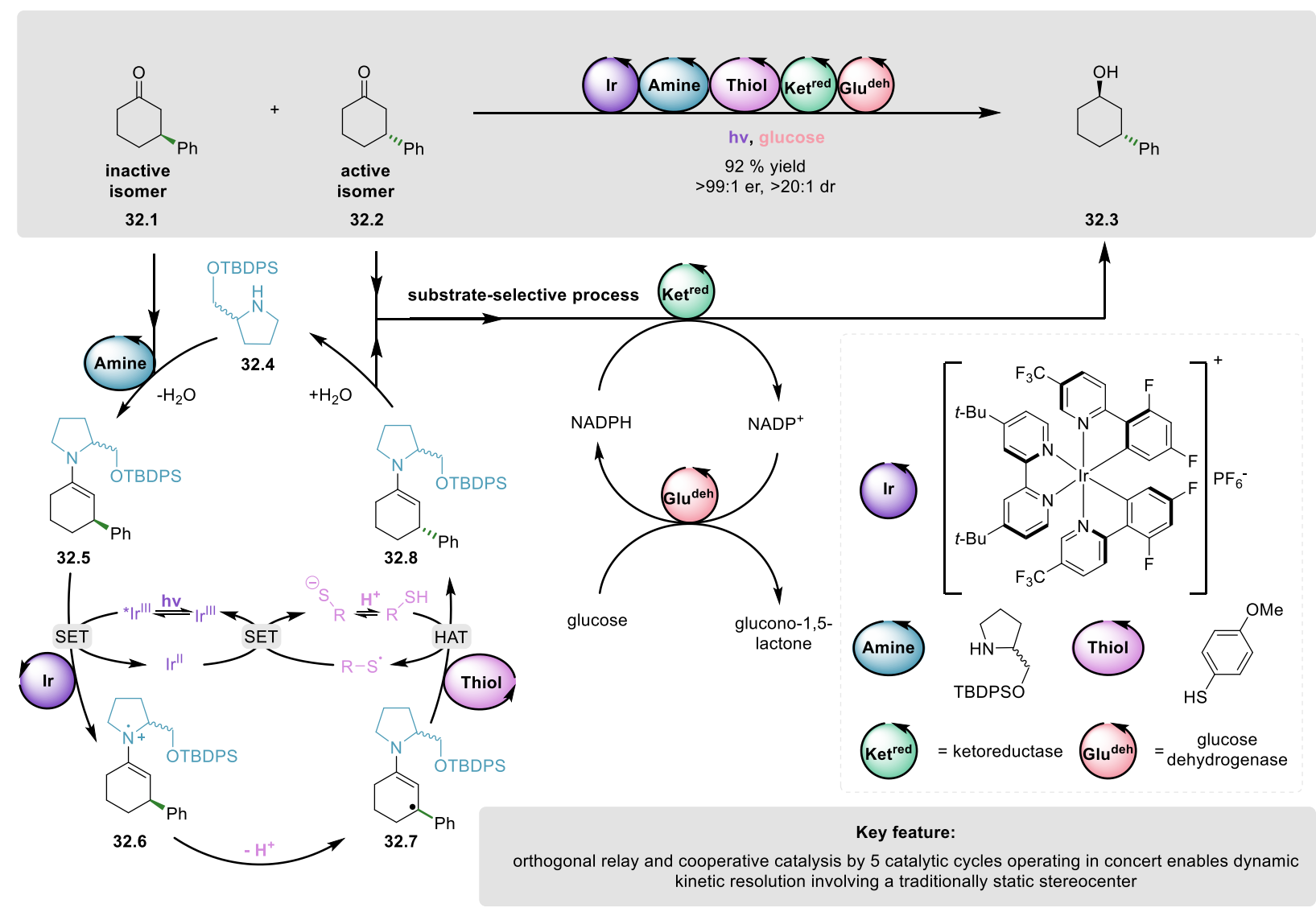

\subsection{Assisted complex systems:}

The possibility to modify the conditions of reaction sequence occurring under multi-catalysis expedites the development of such complex systems, however, at the price of preventing the cooperativity between reactions taking place in different phases of the process. Nevertheless, such 'assisted' systems can substantially simplify synthetic processes, increasing their overall efficiency. Bellow we discuss selected examples of assisted complex systems exploiting either sequential or assisted-relay catalysis along with cooperative, auto- or orthogonal-relay catalysis.

Sequential catalysis with the $2^{\text {nd }}$ step involving cooperative catalysis (3 catalysts) 
Beller $^{137}$ and co-workers developed a triple catalytic system that exploits sequential and cooperative catalysis to directly convert aryl alkynes, anilines, and molecular hydrogen into enantioenriched secondary benzylic amines (Scheme 33). Initially, an Au-catalyst triggers hydroamination of alkyne 33.1 with aniline $\mathbf{3 3 . 2}$ to form an imine, which is subsequently isomerized to ketimine intermediate 33.4. Upon completion, the inert atmosphere is exchanged for hydrogen, Knölker's complex and a chiral phosphoric acid are added, which catalyze cooperative enantioselective reduction of the ketimine intermediate $\mathbf{3 3 . 4}$ to form the benzylamine $\mathbf{3 3 . 3}$ in high yield and enantioselectivity. Notably, the authors showed that the sequence occurs when all reagents and catalysts are present from the start (i.e., under orthogonal-relay and cooperative catalysis). However, in the latter case, the product was formed in lower yield and selectivity than for the sequential approach ( $42 \%$ yield and $49 \%$ ee, versus $80 \%$ yield, and $94 \%$ ee, respectively), indicating that sequential addition prevents the detrimental side reactions. 
Scheme 33. Au-/ Fe-/Brønsted acid-catalyzed sequential hydroamination of alkynes with primary amines followed by cooperative enantioselective hydrogenation

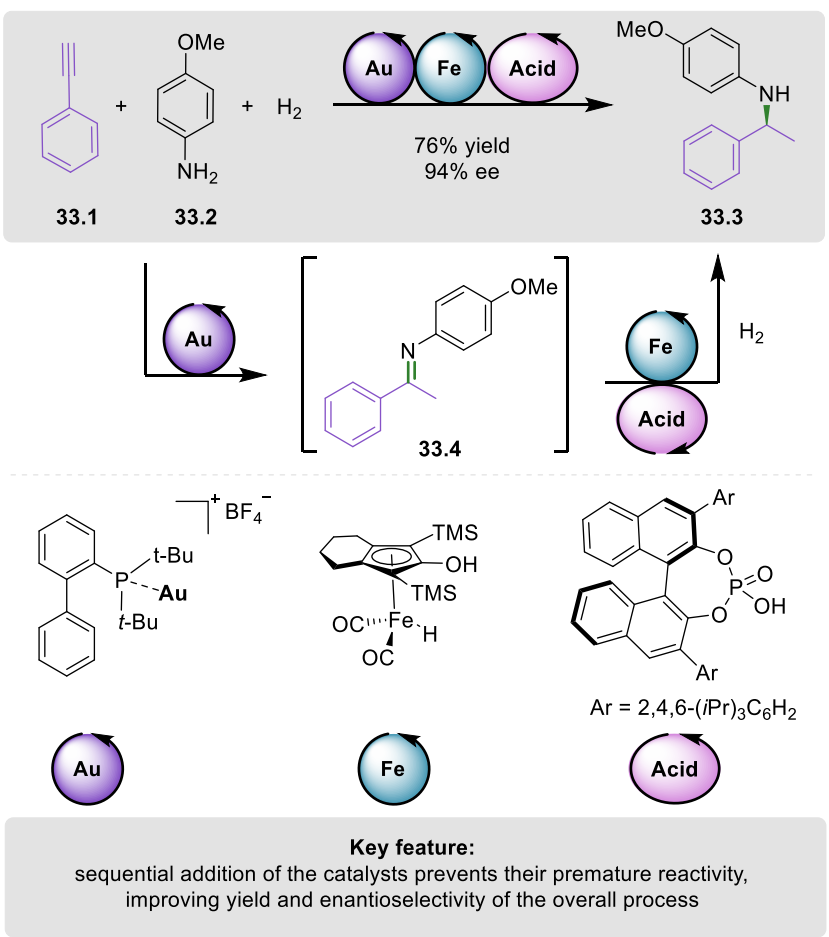

Sequential catalysis with the $2^{\text {nd }}$ step involving both auto-relay and orthogonal relay catalysis (4 reactions with 3 catalysts)

Combining multiple catalytic activities of several catalysts can ideally provide synthetic sequences that transform abundant chemical commodities into value-added building blocks with high atom- and material-efficiency. Huang ${ }^{138}$ and co-workers reported recently a compelling example, where linear alkanes are converted directly into linear alcohols with theoretical $100 \%$ atom efficiency within a triple-catalytic, two-step sequence (Scheme 34). In the first step, alkane 34.1 undergoes acceptorless dehydrogenation to form a mixture of alkenes $\mathbf{3 4 . 3}$ and $\mathbf{3 4 . 4}$ in the presence of an Ir-catalyst. In the second step, the alkene intermediates undergo Rh-catalyzed autorelay isomerization-hydroformylation sequence to form selectively linear aldehyde $\mathbf{3 4 . 5}$, which in 
turn directly undergoes Ru-catalyzed hydrogenation to form the linear alcohol product 34.2. In principle, dihydrogen, the sole by-product of the first step, could be incorporated in the subsequent step, demonstrating the concept of material leveraging in the reaction sequence to improve atomeconomy of the synthesis. It is worth to underscore that all reactions of isomerizationhydroformylation-hydrogenation relay occur in parallel under the same conditions with suitable rates and high chemoselectivity, being the key to form the product in high yield. 
Scheme 34. Ir-/Rh-/Ru-catalyzed sequential dehydrogenation of alkanes followed with orthogonal and auto-relay of olefin isomerization, hydroformylation, and hydrogenation to transform nalkanes to n-alcohols
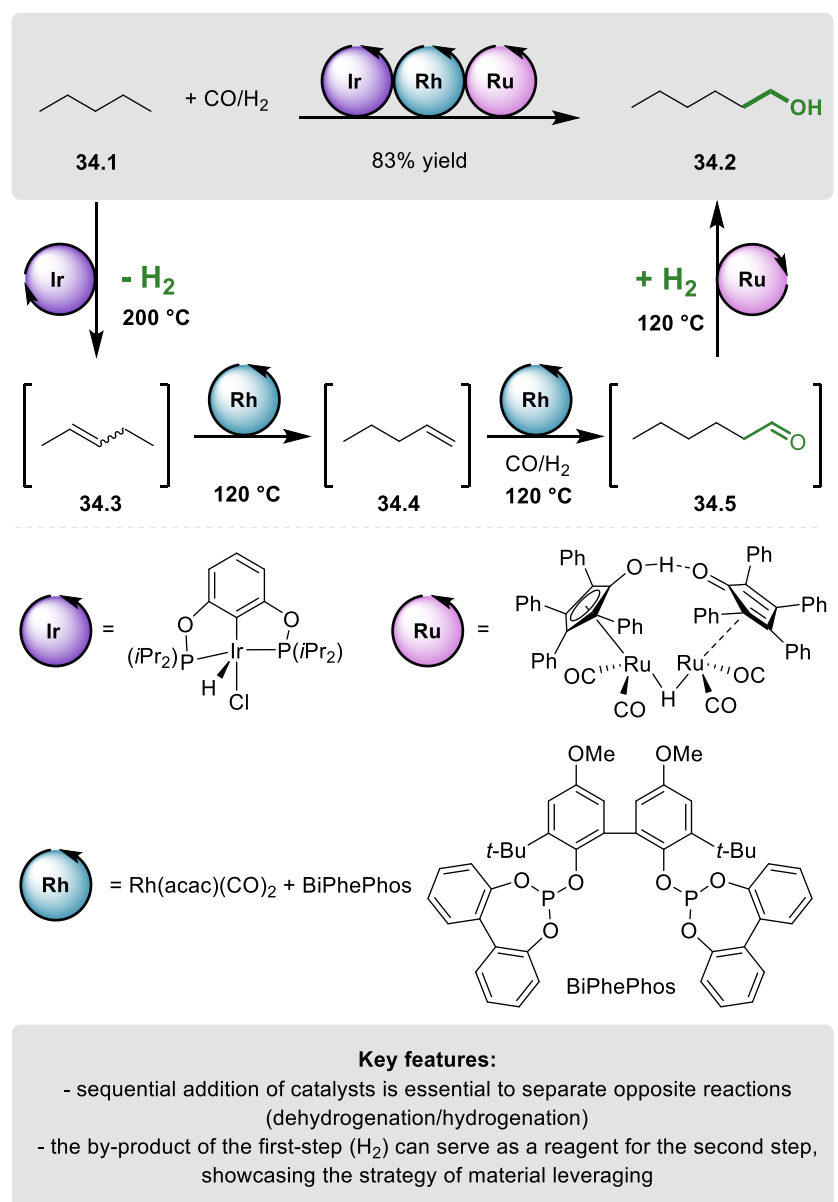

Assisted-relay catalysis with the first step involving orthogonal relay catalysis (3 reactions with 2 catalysts)

Chaładaj $^{139}$ and co-workers reported a four-component Pd-catalyzed perfluoroalkylative arylation of alkynes, which operates through three functionally distinct catalytic cycles, exploiting relay catalysis and temperature-triggered assisted-relay catalysis (Scheme 35). Initially, alkyne 35.1 reacts with perfluoroalkyl iodide 35.2 to form vinyl iodide 35.6 through radical $\mathrm{Pd}^{0} / \mathrm{Pd}^{\mathrm{I}}$ mechanism involving binap-Pd catalyst. The vinyl iodide $\mathbf{3 5 . 6}$ undergoes directly borylation with 
(Bpin) $)_{2}$ to form vinyl boronate ester 35.7 within the orthogonal relay through the $\mathrm{Pd}^{0} / \mathrm{Pd}^{\mathrm{II}}$ mechanism by Xphos-Pd catalyst. Upon completion, the reaction temperature is increased to trigger the Suzuki coupling of the vinyl boronate ester $\mathbf{3 5 . 7}$ with aryl iodide $\mathbf{3 5 . 4}$ to deliver the final product 35.5 .

Scheme 35. A four-substrate orthogonal relay of perfluoroalkylative iodination of alkynes and borylation merged with assisted-relay of temperature-triggered Suzuki cross coupling. Two Pdcatalysts operate three functionally distinct catalytic cycles, merging auto-relay and assisted-relay catalysis
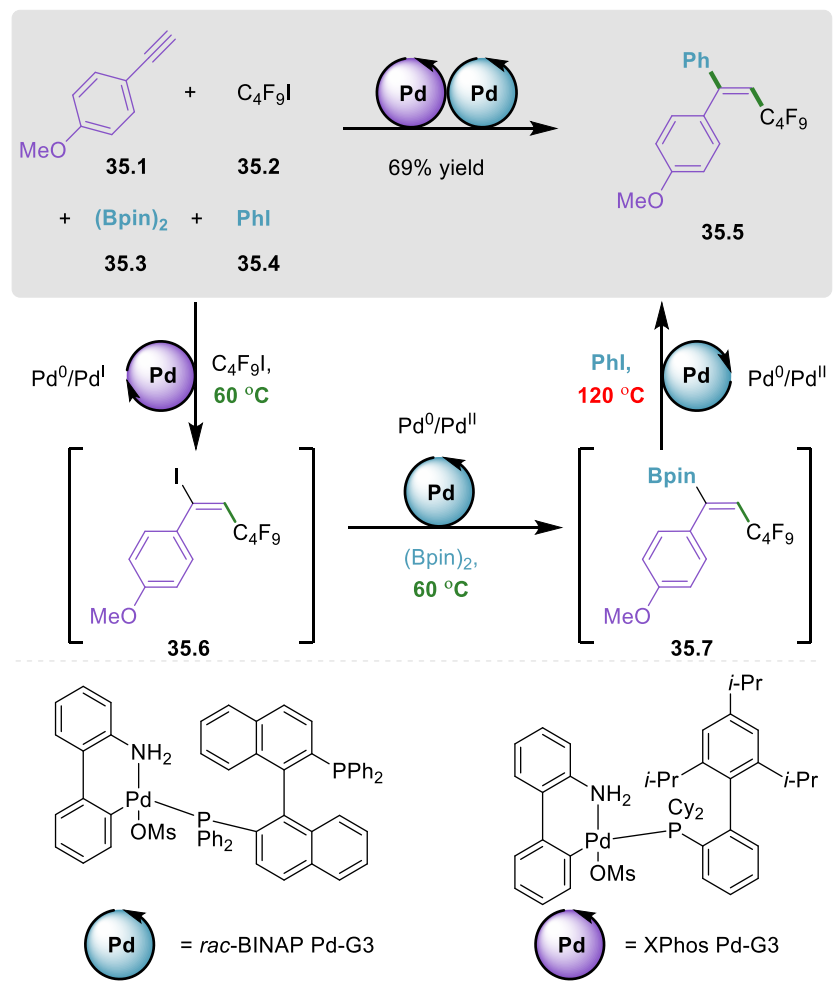

Key feature:

combination of auto- and assited-relay catalysis: temperature increase triggers additional reactivity of the catalyst to promote the last step in the sequence

Assisted-relay catalysis with the $2^{\text {nd }}$ step involving cooperative catalysis (one catalyst acts both cooperatively and non-cooperatively): 
Lautens ${ }^{140}$ and co-workers reported a three-component synthesis of fully substituted triazoles in the presence of two catalysts operating under assisted-relay and cooperative catalysis (Scheme 36). The sequence starts with a substrate-selective $\mathrm{Cu}$-catalyzed cycloaddition of organic azide $\mathbf{3 6 . 1}$ with iodoalkyne 36.2 to form iodotriazole 36.5 , in the presence of another alkyne 36.3 that remains unreactive under the initial conditions. Upon completion, the temperature is increased from room temperature to $85{ }^{\circ} \mathrm{C}$ to trigger the $\mathrm{Pd}-/ \mathrm{Cu}$-catalyzed Sonogashira reaction of iodotriazole intermediate 36.5 with terminal alkyne 36.3 , yielding fully substituted triazole 36.4 in high overall yield. Although the complete sequence can also be executed directly at $85^{\circ} \mathrm{C}$, the product is formed in significantly lower yield (30\% vs. $73 \%$ ). Careful optimization of the Pd-precatalyst, base, and the temperature program was essential to prevent premature side reactions and ordering the catalytic activity. 
Scheme 36. $\mathrm{Cu}-\mathrm{Pd}$-catalyzed temperature-controlled assisted-relay involving cycloaddition and cooperative Sonogashira coupling to obtain fully substituted triazoles

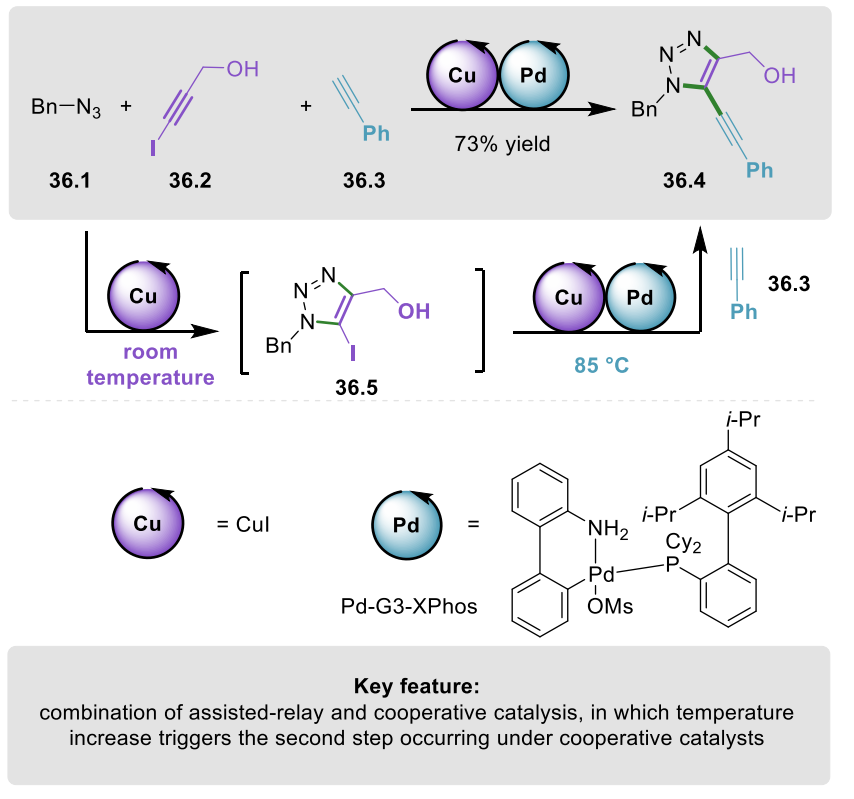

Assisted-relay catalysis with the $1^{\text {st }}$ step involving cooperative catalysis and the $2^{\text {nd }}$ step involving divergent catalysis (one catalyst acts both cooperatively and non-cooperatively):

Catalysts with complementary selectivities, such as stereodivergent selectivity, are attractive in the context of diversity-oriented synthesis. The ability to control the activity of complementary catalysts within a sequence represents an appealing strategy for the divergent synthesis. Lalic ${ }^{141}$ and co-workers recently reported an elegant dual-catalytic system to perform either $(Z)$ - or $(E)$ selective synthesis of vinyl arenes, with the selectivity being controlled by the reaction conditions (Scheme 37). First, a Sonogashira cross-coupling reaction between terminal alkyl alkyne $\mathbf{3 7 . 1}$ and aryl halide 37.2 takes place under cooperative $\mathrm{Pd} / \mathrm{Cu}$-catalysis. Then, alkyne intermediate $\mathbf{3 7 . 5}$ undergoes semireduction under assisted-relay catalysis by either $\mathrm{Cu}$ - or Pd-complex. While the (Z)-selective semi-reduction to furnish $\mathbf{3 7 . 3}$ is promoted by the $\mathrm{Cu}$-complex in the presences of close to stoichiometric amount of methanol as a reductant, the $(E)$-selective semi-reduction to yield 
37.4 selectively is conducted by the Pd-complex in the presence of excess methanol. Overall, the possibility to suppress the activity of the $\mathrm{Cu}$-catalyst by excess methanol provides a convenient handle to direct the overall reaction to form either diastereomer of the product in high selectivity and yield.

Scheme 37. Cu-/Pd-catalyzed reagent-triggered stereo-divergent assisted-relay of cooperative Sonogashira coupling and semireduction to form either the $(Z)$-alkenes under $\mathrm{Cu}$-catalysis or the (E)-alkenes under Pd-catalysis: amount of methanol can divert the activity from $\mathrm{Cu}$ - to $\mathrm{Pd}$-catalyst

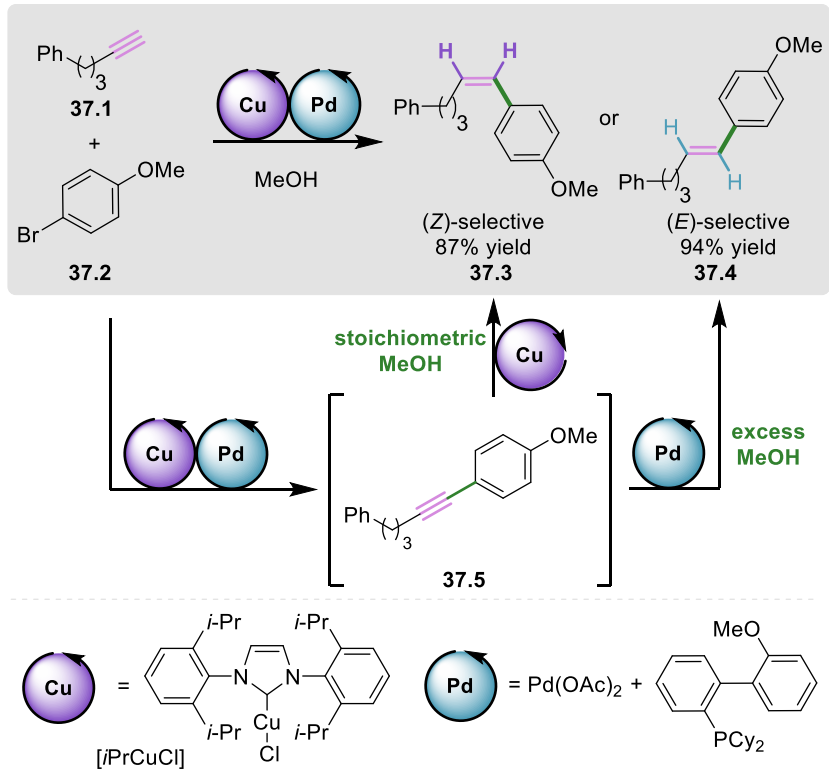

Key feature:

Key feature:
quantity of one reagent $\mathrm{MeOH}$ controls which catalyst engages in the second step of the sequence occurring under cooperative and assisted-relay catalysis, enabling to form either stereoisomer of the product 


\section{Conclusions and outlook}

Driven by the demand for more efficient and sustainable chemical processes, the field of catalysis continues its rapid development. As illustrated in this Perspective article, multi-catalysis emerges not only to outcompete the current synthetic protocols, but most importantly also to bring new 'one-pot' transformations that exhibit outstanding resource efficiency. Remarkably, multicatalytic sequences can undergo through otherwise unfeasible pathways thanks to, for instance, prospectively unstable intermediates being converted as soon as formed. Exemplarily, dualcatalytic systems can enable the direct functionalization of inherently unreactive sites of molecules, thanks to cooperation between reversible catalytic reactions and orthogonal functionalization reactions, opening new valuable short-cuts to the synthetic practitioner.

Despite major challenges that need to be addressed with respect to compatibility issues and catalyst reactivity ordering, recent studies show many competent strategies to address these. For instance, sequential additions of reagents or catalysts, reaction compartmentalization or catalyst encapsulation, proved to be feasible solutions to overcome such incompatibility issues. Likewise, the use of chemical triggers able to induce a change of catalytic activity or stepwise temperature programming of the sequence of reactions, are practical solutions to deliver the correct order of reactions within the targeted sequence. However, these strategies eliminate the advantage of cooperativity between reactions in the sequence, limiting the full potential of multi-catalytic systems. Therefore, novel strategies that address these challenges in situ need to be developed. Recent examples in the literature show that mimicking the features of enzymes, that is, (i) allosteric regulation, (ii) substrate-selective catalysis, or (iii) substrate channeling between consecutive 
catalytic sites, are plausible ways to solve those issues while maintaining the cooperativity between reactions.

In addition, a fundamental change in approach has to be made in order to improve the development of such complex multi-catalytic systems and to study its emergent properties. In this context, suitable catalysts able to perform with excellent chemo- and reaction-selectivity within a complex mixture are most likely to be different than those performing best in single reaction environments. Linear screening of different parameters for such multivariate systems is rather impractical. Therefore, new strategies to design, analyze and validate the development of such systems are required. Overall, although many challenges remain open, the prospects of multicatalysis are vast. We envision that future studies will enable the rational design of processes, in which multiple catalysts are orchestrated precisely to transform simple starting materials into valuable products within complex systems of interconnected reactions.

\section{AUTHOR INFORMATION}

\section{Corresponding Author}

* E-mail: dydio@unistra.fr

\section{Author Contributions}

The manuscript was written through contributions of all authors. All authors have given approval to the final version of the manuscript.

† SM and LV contributed equally.

\section{ACKNOWLEDGMENT}


This work was financially supported by the European Research Council (ERC StG „Reverse\&Cat“ \#804106), the French National Research Agency (ANR IdEx, ANR LabEx „Chemistry of Complex Systems“, and a PhD fellowship for BL through the Programme d' Investissement d' Avenir under contract 17-EURE-0016), and the Frontier Research in Chemistry Foundation (a Solvay $\mathrm{PhD}$ fellowship for SM).

\section{REFERENCES}

(1) Bartholomew, C. H.; Farrauto, R. J. Fundamentals of Industrial Catalytic Processes; John Wiley \& Sons, Inc.: Hoboken, NJ, USA, 2005. https://doi.org/10.1002/9780471730071.

(2) Sancheti, S. P.; Urvashi; Shah, M. P.; Patil, N. T. Ternary Catalysis: A Stepping Stone toward Multicatalysis. ACS Catal. 2020, $10 \quad$ (5), $3462-3489$. https://doi.org/10.1021/acscatal.9b04000.

(3) Kim, U. Bin; Jung, D. J.; Jeon, H. J.; Rathwell, K.; Lee, S. Synergistic Dual Transition Metal Catalysis. Chem. Rev. 2020, 120 (24), 13382-13433. https://doi.org/10.1021/acs.chemrev.0c00245.

(4) Ambrosini, L. M.; Lambert, T. H. Multicatalysis: Advancing Synthetic Efficiency and Inspiring Discovery. ChemCatChem. 2010. https://doi.org/10.1002/cctc.200900323.

(5) Patil, N. T.; Shinde, V. S.; Gajula, B. A One-Pot Catalysis: The Strategic Classification with Some Recent Examples. Org. Biomol. Chem. 2012, 10 (2), 211-224. https://doi.org/10.1039/C1OB06432K.

(6) Quintard, A. Iron-Based Multi-Catalysis: Eco-Compatible Alternative for Complex Molecules Synthesis. Chemistry - A European Journal. 2021. 
https://doi.org/10.1002/chem.202002092.

(7) Lee, J. M.; Na, Y.; Han, H.; Chang, S. Cooperative Multi-Catalyst Systems for One-Pot Organic Transformations. Chem. Soc. Rev. 2004. https://doi.org/10.1039/b309033g.

(8) Shao, Z.; Zhang, H. Combining Transition Metal Catalysis and Organocatalysis: A Broad New Concept for Catalysis. Chem. Soc. Rev. 2009. https://doi.org/10.1039/b901258n.

(9) Rueping, M.; Koenigs, R. M.; Atodiresei, I. Unifying Metal and Brønsted Acid CatalysisConcepts, Mechanisms, and Classifications. Chem. - A Eur. J. 2010. https://doi.org/10.1002/chem.201001140.

(10) Lohr, T. L.; Marks, T. J. Orthogonal Tandem Catalysis. Nat. Chem. 2015, 7 (6), 477-482. https://doi.org/10.1038/nchem.2262.

(11) Fogg, D. E.; dos Santos, E. N. Tandem Catalysis: A Taxonomy and Illustrative Review. Coord. Chem. Rev. 2004, $248 \quad$ (21-24), 2365-2379. https://doi.org/10.1016/j.ccr.2004.05.012.

(12) Chapman, C. J.; Frost, C. G. Tandem and Domino Catalytic Strategies for Enantioselective Synthesis. Synthesis. 2007. https://doi.org/10.1055/s-2006-950379.

(13) Hopkinson, M. N.; Sahoo, B.; Li, J. L.; Glorius, F. Dual Catalysis Sees the Light: Combining Photoredox with Organo-, Acid, and Transition-Metal Catalysis. Chemistry - A European Journal. 2014. https://doi.org/10.1002/chem.201304823.

(14) Hayashi, Y. Pot Economy and One-Pot Synthesis. Chemical Science. 2016. https://doi.org/10.1039/c5sc02913a. 
(15) Yadav, R. N.; Srivastava, A. K.; Banik, B. K. One-Pot Strategy: A Highly Economical Tool in Organic Synthesis and Medicinal Chemistry. In Green Approaches in Medicinal Chemistry for Sustainable Drug Design; 2020. https://doi.org/10.1016/b978-0-12-8175927.00010-1.

(16) Allen, A. E.; MacMillan, D. W. C. Synergistic Catalysis: A Powerful Synthetic Strategy for New Reaction Development. Chem. Sci. 2012, $3 \quad$ (3), 633. https://doi.org/10.1039/c2sc00907b.

(17) Romiti, F.; del Pozo, J.; Paioti, P. H. S.; Gonsales, S. A.; Li, X.; Hartrampf, F. W. W.; Hoveyda, A. H. Different Strategies for Designing Dual-Catalytic Enantioselective Processes: From Fully Cooperative to Non-Cooperative Systems. J. Am. Chem. Soc. 2019, 141 (45), 17952-17961. https://doi.org/10.1021/jacs.9b05464.

(18) Inamdar, S. M.; Shinde, V. S.; Patil, N. T. Enantioselective Cooperative Catalysis. Org. Biomol. Chem. 2015, 13 (30), 8116-8162. https://doi.org/10.1039/C5OB00986C.

(19) Pellissier, H. Recent Developments in Enantioselective Multicatalyzed Tandem Reactions. Advanced Synthesis and Catalysis. 2020. https://doi.org/10.1002/adsc.202000210.

(20) Rudroff, F.; Mihovilovic, M. D.; Gröger, H.; Snajdrova, R.; Iding, H.; Bornscheuer, U. T. Opportunities and Challenges for Combining Chemo- and Biocatalysis. Nat. Catal. 2018, 1 (1), 12-22. https://doi.org/10.1038/s41929-017-0010-4.

(21) Galván, A.; Fañanás, F. J.; Rodríguez, F. Multicomponent and Multicatalytic Reactions - A Synthetic Strategy Inspired by Nature. Eur. J. Inorg. Chem. 2016. https://doi.org/10.1002/ejic.201501287. 
(22) Hwang, E. T.; Lee, S. Multienzymatic Cascade Reactions via Enzyme Complex by Immobilization. $\quad$ ACS $\quad$ Catal. 2019, $\quad 9 \quad$ (5), $\quad$ 4402-4425. https://doi.org/10.1021/acscatal.8b04921.

(23) Ji, Q.; Wang, B.; Tan, J.; Zhu, L.; Li, L. Immobilized Multienzymatic Systems for Catalysis of Cascade Reactions. Process Biochem. 2016, 51 (9), 1193-1203. https://doi.org/https://doi.org/10.1016/j.procbio.2016.06.004.

(24) Sperl, J. M.; Sieber, V. Multienzyme Cascade Reactions-Status and Recent Advances. ACS Catal. 2018, 8 (3), 2385-2396. https://doi.org/10.1021/acscatal.7b03440.

(25) Ricca, E.; Brucher, B.; Schrittwieser, J. H. Multi-Enzymatic Cascade Reactions: Overview and Perspectives. Adv. Synth. Catal. 2011, 353 (13), 2239-2262. https://doi.org/10.1002/adsc.201100256.

(26) Tietze, L. F.; Brasche, G.; Gericke, K. M. Domino Reactions in Organic Synthesis. In Domino Reactions in Organic Synthesis; Wiley: Weinheim, 2006; pp 1-541. https://doi.org/10.1002/9783527609925.

(27) Kim, D.-S.; Park, W.-J.; Jun, C.-H. Metal-Organic Cooperative Catalysis in C-H and C-C Bond Activation. Chem. Rev. 2017, $117 \quad$ (13), 8977-9015. https://doi.org/10.1021/acs.chemrev.6b00554.

(28) Afewerki, S.; Córdova, A. Combinations of Aminocatalysts and Metal Catalysts: A Powerful Cooperative Approach in Selective Organic Synthesis. Chem. Rev. 2016, 116(22), 13512-13570. https://doi.org/10.1021/acs.chemrev.6b00226.

(29) Peters, R. Cooperative Catalysis; Peters, R., Ed.; Wiley: Weinheim, 2015. 
https://doi.org/10.1002/9783527681020.

(30) Krautwald, S.; Sarlah, D.; Schafroth, M. A.; Carreira, E. M. Enantio- and Diastereodivergent Dual Catalysis: -Allylation of Branched Aldehydes. Science 2013, 340 (6136), 1065-1068. https://doi.org/10.1126/science.1237068.

(31) Krautwald, S.; Schafroth, M. A.; Sarlah, D.; Carreira, E. M. Stereodivergent $\alpha$-Allylation of Linear Aldehydes with Dual Iridium and Amine Catalysis. J. Am. Chem. Soc. 2014, 136 (8), 3020-3023. https://doi.org/10.1021/ja5003247.

(32) Sandmeier, T.; Krautwald, S.; Zipfel, H. F.; Carreira, E. M. Stereodivergent Dual Catalytic $\alpha$-Allylation of Protected $\alpha$-Amino- and $\alpha$-Hydroxyacetaldehydes. Angew. Chemie Int. Ed. 2015, 54 (48), 14363-14367. https://doi.org/10.1002/anie.201506933.

(33) He, R.; Huo, X.; Zhao, L.; Wang, F.; Jiang, L.; Liao, J.; Zhang, W. Stereodivergent Pd/Cu Catalysis for the Dynamic Kinetic Asymmetric Transformation of Racemic Unsymmetrical 1,3-Disubstituted Allyl Acetates. J. Am. Chem. Soc. 2020, 142 (18), 8097-8103. https://doi.org/10.1021/jacs.0c02150.

(34) Huo, X.; Zhang, J.; Fu, J.; He, R.; Zhang, W. Ir/Cu Dual Catalysis: Enantio- and Diastereodivergent Access to $\alpha, \alpha$-Disubstituted $\alpha$-Amino Acids Bearing Vicinal Stereocenters. J. Am. Chem. Soc. 2018, $140 \quad$ (6), 2080-2084. https://doi.org/10.1021/jacs.8b00187.

(35) Jiang, X.; Boehm, P.; Hartwig, J. F. Stereodivergent Allylation of Azaaryl Acetamides and Acetates by Synergistic Iridium and Copper Catalysis. J. Am. Chem. Soc. 2018, 140 (4), 1239-1242. https://doi.org/10.1021/jacs.7b12824. 
(36) Wei, L.; Zhu, Q.; Xu, S.-M.; Chang, X.; Wang, C.-J. Stereodivergent Synthesis of $\alpha, \alpha-$ Disubstituted $\alpha$-Amino Acids via Synergistic Cu/Ir Catalysis. J. Am. Chem. Soc. 2018, 140 (4), 1508-1513. https://doi.org/10.1021/jacs.7b12174.

(37) He, Z.-T.; Jiang, X.; Hartwig, J. F. Stereodivergent Construction of Tertiary Fluorides in Vicinal Stereogenic Pairs by Allylic Substitution with Iridium and Copper Catalysts. J. Am. Chem. Soc. 2019, 141 (33), 13066-13073. https://doi.org/10.1021/jacs.9b04440.

(38) Xu, S.-M.; Wei, L.; Shen, C.; Xiao, L.; Tao, H.-Y.; Wang, C.-J. Stereodivergent Assembly of Tetrahydro- $\gamma$-Carbolines via Synergistic Catalytic Asymmetric Cascade Reaction. Nat. Commun. 2019, 10 (1), 5553. https://doi.org/10.1038/s41467-019-13529-z.

(39) Zhang, Q.; Yu, H.; Shen, L.; Tang, T.; Dong, D.; Chai, W.; Zi, W. Stereodivergent Coupling of 1,3-Dienes with Aldimine Esters Enabled by Synergistic Pd and Cu Catalysis. J. Am. Chem. Soc. 2019, 141 (37), 14554-14559. https://doi.org/10.1021/jacs.9b07600.

(40) Sandmeier, T.; Carreira, E. M. Enantioselective Iridium-Catalyzed $\alpha$-Allylation with Aqueous Solutions of Acetaldehyde. Org. Lett. 2020, 22 (3), 1135-1138. https://doi.org/10.1021/acs.orglett.9b04658.

(41) Singha, S.; Serrano, E.; Mondal, S.; Daniliuc, C. G.; Glorius, F. Diastereodivergent Synthesis of Enantioenriched $\alpha, \beta$-Disubstituted $\gamma$-Butyrolactones via Cooperative NHeterocyclic Carbene and Ir Catalysis. Nat. Catal. 2020, 3 (1), 48-54. https://doi.org/10.1038/s41929-019-0387-3.

(42) Zhang, M.-M.; Wang, Y.-N.; Wang, B.-C.; Chen, X.-W.; Lu, L.-Q.; Xiao, W.-J. Synergetic Iridium and Amine Catalysis Enables Asymmetric [4+2] Cycloadditions of Vinyl 
Aminoalcohols with Carbonyls. Nat. Commun. 2019, $10 \quad$ (1), 2716. https://doi.org/10.1038/s41467-019-10674-3.

(43) Kim, B.; Kim, Y.; Lee, S. Y. Stereodivergent Carbon-Carbon Bond Formation between Iminium and Enolate Intermediates by Synergistic Organocatalysis. J. Am. Chem. Soc. 2021, 143 (1), 73-79. https://doi.org/10.1021/jacs.0c11077.

(44) Tellis, J. C.; Primer, D. N.; Molander, G. A. Single-Electron Transmetalation in Organoboron Cross-Coupling by Photoredox/Nickel Dual Catalysis. Science 2014, 345 (6195), 433-436. https://doi.org/10.1126/science.1253647.

(45) Twilton, J.; Le, C.; Zhang, P.; Shaw, M. H.; Evans, R. W.; MacMillan, D. W. C. The Merger of Transition Metal and Photocatalysis. Nat. Rev. Chem. 2017, 1 (7), 0052. https://doi.org/10.1038/s41570-017-0052.

(46) Zuo, Z.; Ahneman, D. T.; Chu, L.; Terrett, J. A.; Doyle, A. G.; MacMillan, D. W. C. Merging Photoredox with Nickel Catalysis: Coupling of -Carboxyl Sp3-Carbons with Aryl Halides. Science 2014, 345 (6195), 437-440. https://doi.org/10.1126/science.1255525.

(47) Jouffroy, M.; Primer, D. N.; Molander, G. A. Base-Free Photoredox/Nickel Dual-Catalytic Cross-Coupling of Ammonium Alkylsilicates. J. Am. Chem. Soc. 2016, 138 (2), 475-478. https://doi.org/10.1021/jacs.5b10963.

(48) Nakajima, K.; Nojima, S.; Nishibayashi, Y. Nickel- and Photoredox-Catalyzed CrossCoupling Reactions of Aryl Halides with 4-Alkyl-1,4-Dihydropyridines as Formal Nucleophilic Alkylation Reagents. Angew. Chemie Int. Ed. 2016, 55 (45), 14106-14110. https://doi.org/10.1002/anie.201606513. 
(49) Milligan, J. A.; Phelan, J. P.; Badir, S. O.; Molander, G. A. Alkyl Carbon-Carbon Bond Formation by Nickel/Photoredox Cross-Coupling. Angew. Chemie Int. Ed. 2019, 58 (19), 6152-6163. https://doi.org/10.1002/anie.201809431.

(50) Shaw, M. H.; Shurtleff, V. W.; Terrett, J. A.; Cuthbertson, J. D.; MacMillan, D. W. C. Native Functionality in Triple Catalytic Cross-Coupling: Sp3 C-H Bonds as Latent Nucleophiles. $\quad$ Science $\quad$ 2016, $352 \quad$ (6291), https://doi.org/10.1126/science.aaf6635.

(51) Le, C.; Liang, Y.; Evans, R. W.; Li, X.; MacMillan, D. W. C. Selective Sp3 C-H Alkylation via Polarity-Match-Based Cross-Coupling. Nature 2017, 547 (7661), 79-83. https://doi.org/10.1038/nature22813.

(52) Zhang, X.; MacMillan, D. W. C. Direct Aldehyde C-H Arylation and Alkylation via the Combination of Nickel, Hydrogen Atom Transfer, and Photoredox Catalysis. J. Am. Chem. Soc. 2017, 139 (33), 11353-11356. https://doi.org/10.1021/jacs.7b07078.

(53) Heitz, D. R.; Tellis, J. C.; Molander, G. A. Photochemical Nickel-Catalyzed C-H Arylation: Synthetic Scope and Mechanistic Investigations. J. Am. Chem. Soc. 2016, 138 (39), 1271512718. https://doi.org/10.1021/jacs.6b04789.

(54) Shields, B. J.; Doyle, A. G. Direct C(Sp 3 )-H Cross Coupling Enabled by Catalytic Generation of Chlorine Radicals. J. Am. Chem. Soc. 2016, 138 (39), 12719-12722. https://doi.org/10.1021/jacs.6b08397.

(55) Perry, I. B.; Brewer, T. F.; Sarver, P. J.; Schultz, D. M.; DiRocco, D. A.; MacMillan, D. W. C. Direct Arylation of Strong Aliphatic C-H Bonds. Nature 2018, 560 (7716), 70-75. 
https://doi.org/10.1038/s41586-018-0366-x.

(56) Lee, G. S.; Won, J.; Choi, S.; Baik, M.-H.; Hong, S. H. Synergistic Activation of Amides and Hydrocarbons for Direct $\mathrm{C}(\mathrm{Sp} 3$ ) $-\mathrm{H}$ Acylation Enabled by Metallaphotoredox Catalysis. Angew. Chemie Int. Ed. 2020, 59 (39), 16933-16942. https://doi.org/10.1002/anie.202004441.

(57) Thullen, S. M.; Treacy, S. M.; Rovis, T. Regioselective Alkylative Cross-Coupling of Remote Unactivated C( Sp 3 )-H Bonds. J. Am. Chem. Soc. 2019, 141 (36), 14062-14067. https://doi.org/10.1021/jacs.9b07014.

(58) Cao, H.; Kuang, Y.; Shi, X.; Wong, K. L.; Tan, B. B.; Kwan, J. M. C.; Liu, X.; Wu, J. Photoinduced Site-Selective Alkenylation of Alkanes and Aldehydes with Aryl Alkenes. Nat. Commun. 2020, 11 (1), 1956. https://doi.org/10.1038/s41467-020-15878-6.

(59) Shaw, M. H.; Twilton, J.; MacMillan, D. W. C. Photoredox Catalysis in Organic Chemistry. J. Org. Chem. 2016, 81 (16), 6898-6926. https://doi.org/10.1021/acs.joc.6b01449.

(60) Hamilton, D. S.; Nicewicz, D. A. Direct Catalytic Anti-Markovnikov Hydroetherification of Alkenols. J. Am. Chem. Soc. 2012, 134 (45), 18577-18580. https://doi.org/10.1021/ja309635w.

(61) Wilger, D. J.; Grandjean, J.-M. M.; Lammert, T. R.; Nicewicz, D. A. The Direct AntiMarkovnikov Addition of Mineral Acids to Styrenes. Nat. Chem. 2014, 6 (8), 720-726. https://doi.org/10.1038/nchem.2000.

(62) Margrey, K. A.; Nicewicz, D. A. A General Approach to Catalytic Alkene AntiMarkovnikov Hydrofunctionalization Reactions via Acridinium Photoredox Catalysis. Acc. 
Chem. Res. 2016, 49 (9), 1997-2006. https://doi.org/10.1021/acs.accounts.6b00304.

(63) Perkowski, A. J.; Nicewicz, D. A. Direct Catalytic Anti-Markovnikov Addition of Carboxylic Acids to Alkenes. J. Am. Chem. Soc. 2013, 135 (28), 10334-10337. https://doi.org/10.1021/ja4057294.

(64) Nguyen, T. M.; Nicewicz, D. A. Anti-Markovnikov Hydroamination of Alkenes Catalyzed by an Organic Photoredox System. J. Am. Chem. Soc. 2013, 135 (26), 9588-9591. https://doi.org/10.1021/ja4031616.

(65) Nguyen, T. M.; Manohar, N.; Nicewicz, D. A. Anti -Markovnikov Hydroamination of Alkenes Catalyzed by a Two-Component Organic Photoredox System: Direct Access to Phenethylamine Derivatives. Angew. Chemie Int. Ed. 2014, 53 (24), 6198-6201. https://doi.org/10.1002/anie.201402443.

(66) Zeller, M. A.; Riener, M.; Nicewicz, D. A. Butyrolactone Synthesis via Polar Radical Crossover Cycloaddition Reactions: Diastereoselective Syntheses of Methylenolactocin and Protolichesterinic Acid. Org. Lett. 2014, 16 (18), 4810-4813. https://doi.org/10.1021/ol5022993.

(67) Cavanaugh, C. L.; Nicewicz, D. A. Synthesis of $\alpha$-Benzyloxyamino- $\gamma$-Butyrolactones via a Polar Radical Crossover Cycloaddition Reaction. Org. Lett. 2015, 17 (24), 6082-6085. https://doi.org/10.1021/acs.orglett.5b03113.

(68) Gesmundo, N. J.; Grandjean, J.-M. M.; Nicewicz, D. A. Amide and Amine Nucleophiles in Polar Radical Crossover Cycloadditions: Synthesis of $\gamma$-Lactams and Pyrrolidines. Org. Lett. 2015, 17 (5), 1316-1319. https://doi.org/10.1021/acs.orglett.5b00316. 
(69) Wu, F.; Wang, L.; Chen, J.; Nicewicz, D. A.; Huang, Y. Direct Synthesis of Polysubstituted Aldehydes via Visible-Light Catalysis. Angew. Chemie Int. Ed. 2018, 57 (8), 2174-2178. https://doi.org/10.1002/anie.201712384.

(70) Romero, N. A.; Margrey, K. A.; Tay, N. E.; Nicewicz, D. A. Site-Selective Arene C-H Amination via Photoredox Catalysis. Science 2015, 349 (6254), 1326-1330. https://doi.org/10.1126/science.aac9895.

(71) Chen, W.; Huang, Z.; Tay, N. E. S.; Giglio, B.; Wang, M.; Wang, H.; Wu, Z.; Nicewicz, D. A.; Li, Z. Direct Arene C-H Fluorination with 18 F - via Organic Photoredox Catalysis. Science 2019, 364 (6446), 1170-1174. https://doi.org/10.1126/science.aav7019.

(72) Ohmatsu, K.; Suzuki, R.; Furukawa, Y.; Sato, M.; Ooi, T. Zwitterionic 1,2,3-Triazolium Amidate as a Catalyst for Photoinduced Hydrogen-Atom Transfer Radical Alkylation. ACS Catal. 2020, 10 (4), 2627-2632. https://doi.org/10.1021/acscatal.9b04491.

(73) Shin, N. Y.; Ryss, J. M.; Zhang, X.; Miller, S. J.; Knowles, R. R. Light-Driven Deracemization Enabled by Excited-State Electron Transfer. Science 2019, 366 (6463), 364-369. https://doi.org/10.1126/science.aay2204.

(74) Wang, Y.; Carder, H. M.; Wendlandt, A. E. Synthesis of Rare Sugar Isomers through SiteSelective Epimerization. Nature 2020, $578 \quad$ (7795), $\quad 403-408$. https://doi.org/10.1038/s41586-020-1937-1.

(75) Pellissier, H. Stereocontrolled Domino Reactions. Chem. Rev. 2013, 113 (1), 442-524. https://doi.org/10.1021/cr300271k.

(76) Grossmann, A.; Enders, D. N-Heterocyclic Carbene Catalyzed Domino Reactions. Angew. 
Chemie Int. Ed. 2012, 51 (2), 314-325. https://doi.org/10.1002/anie.201105415.

(77) Pellissier, H. Recent Developments in Enantioselective Metal-Catalyzed Domino Reactions. Advanced Synthesis and Catalysis. 2016, pp 2194-2259. https://doi.org/10.1002/adsc.201600462.

(78) Clavier, H.; Pellissier, H. Recent Developments in Enantioselective Metal-Catalyzed Domino Reactions. Adv. Synth. Catal. 2012, 354 (18), 3347-3403. https://doi.org/10.1002/adsc.201200254.

(79) Pellissier, H. Recent Developments in Enantioselective Metal-Catalyzed Domino $\begin{array}{lllllll}\text { Reactions. } & \text { Adv. Synth. } & \text { Catal. 2019, } 361 \quad \text { (8), 1733-1755. }\end{array}$ https://doi.org/10.1002/adsc.201801371.

(80) Chanda, T.; Zhao, J. C. G. Recent Progress in Organocatalytic Asymmetric Domino Transformations. Adv. Synth. Catal. 2018, $360 \quad$ (1), 2-79. https://doi.org/10.1002/adsc.201701059.

(81) Tietze, L. F. Domino Reactions. In Domino Reactions: Concepts for Efficient Organic Synthesis; Tietze, L. F., Ed.; Wiley-VCH Verlag GmbH \& Co. KGaA: Weinheim, Germany, 2014; pp 1-621. https://doi.org/10.1002/9783527671304.

(82) Trost, B. M.; Min, C. Total Synthesis of Terpenes via Palladium-Catalysed Cyclization Strategy. Nat. Chem. 2020, 12 (6), 568-573. https://doi.org/10.1038/s41557-020-0439-y.

(83) Trost, B. M.; Shi, Y. Palladium-Catalyzed Cyclizations of Polyenynes. A Palladium Zipper. J. Am. Chem. Soc. 1993, 115 (21), 9421-9438. https://doi.org/10.1021/ja00074a008.

(84) Whyte, A.; Torelli, A.; Mirabi, B.; Prieto, L.; Rodríguez, J. F.; Lautens, M. Cobalt- 
Catalyzed Enantioselective Hydroarylation of 1,6-Enynes. J. Am. Chem. Soc. 2020, 142 (20), 9510-9517. https://doi.org/10.1021/jacs.0c03246.

(85) Takahashi, K.; Yamashita, M.; Nozaki, K. Tandem Hydroformylation/Hydrogenation of Alkenes to Normal Alcohols Using Rh/Ru Dual Catalyst or Ru Single Component Catalyst. J. Am. Chem. Soc. 2012, 134 (45), 18746-18757. https://doi.org/10.1021/ja307998h.

(86) Takahashi, K.; Yamashita, M.; Ichihara, T.; Nakano, K.; Nozaki, K. High-Yielding Tandem Hydroformylation/Hydrogenation of a Terminal Olefin to Produce a Linear Alcohol Using a $\mathrm{Rh} / \mathrm{Ru}$ Dual Catalyst System. Angew. Chemie Int. Ed. 2010, 49 (26), 4488-4490. https://doi.org/10.1002/anie.201001327.

(87) Ahmed, M.; Seayad, A. M.; Jackstell, R.; Beller, M. Amines Made Easily: A Highly Selective Hydroaminomethylation of Olefins. J. Am. Chem. Soc. 2003, 125 (34), 1031110318. https://doi.org/10.1021/ja030143w.

(88) Hanna, S.; Holder, J. C.; Hartwig, J. F. A Multicatalytic Approach to the Hydroaminomethylation of A-Olefins. Angew. Chemie Int. Ed. 2019, 58 (11), 3368-3372. https://doi.org/10.1002/anie.201811297.

(89) Jia, Z.-J.; Shan, G.; Daniliuc, C. G.; Antonchick, A. P.; Waldmann, H. Enantioselective Synthesis of the Spirotropanyl Oxindole Scaffold through Bimetallic Relay Catalysis. Angew. Chemie Int. Ed. 2018, $57 \quad$ (44), 14493-14497. https://doi.org/10.1002/anie.201712882.

(90) Yu, Q.; Fu, Y.; Huang, J.; Qin, J.; Zuo, H.; Wu, Y.; Zhong, F. Enantioselective Oxidative Phenol-Indole [3 + 2] Coupling Enabled by Biomimetic Mn(Iii)/Brønsted Acid Relay 
Catalysis. ACS Catal. 2019, 9 (8), 7285-7291. https://doi.org/10.1021/acscatal.9b01734.

(91) Denard, C. A.; Bartlett, M. J.; Wang, Y.; Lu, L.; Hartwig, J. F.; Zhao, H. Development of a One-Pot Tandem Reaction Combining Ruthenium-Catalyzed Alkene Metathesis and Enantioselective Enzymatic Oxidation to Produce Aryl Epoxides. ACS Catal. 2015, 5 (6), 3817-3822. https://doi.org/10.1021/acscatal.5b00533.

(92) Corma, A.; Navas, J.; Sabater, M. J. Advances in One-Pot Synthesis through Borrowing Hydrogen Catalysis. Chem. Rev. 2018, 118 (4), 1410-1459. https://doi.org/10.1021/acs.chemrev.7b00340.

(93) Goldman, A. S. Catalytic Alkane Metathesis by Tandem Alkane Dehydrogenation-Olefin Metathesis. Science 2006, 312 (5771), 257-261. https://doi.org/10.1126/science.1123787.

(94) Lichosyt, D.; Zhang, Y.; Hurej, K.; Dydio, P. Dual-Catalytic Transition Metal Systems for Functionalization of Unreactive Sites of Molecules. Nat. Catal. 2019, 2 (2), 114-122. https://doi.org/10.1038/s41929-018-0207-1.

(95) Quintard, A.; Constantieux, T.; Rodriguez, J. An Iron/Amine-Catalyzed Cascade Process for the Enantioselective Functionalization of Allylic Alcohols. Angew. Chemie Int. Ed. 2013, 52 (49), 12883-12887. https://doi.org/https://doi.org/10.1002/anie.201307295.

(96) Roudier, M.; Constantieux, T.; Quintard, A.; Rodriguez, J. Triple Iron/Copper/Iminium Activation for the Efficient Redox Neutral Catalytic Enantioselective Functionalization of Allylic Alcohols. ACS Catal. 2016, $6 \quad$ (8), 5236-5244. https://doi.org/10.1021/acscatal.6b01102.

(97) Black, P. J.; Harris, W.; Williams, J. M. J. Catalytic Electronic Activation: Indirect Addition 
of Nucleophiles to an Allylic Alcohol This Work Was Supported by the University of Bath and Roche Discovery (P.J.B.). Angew. Chemie Int. Ed. 2001, 40 (23), 4475. https://doi.org/10.1002/1521-3773(20011203)40:23<4475::AID-ANIE4475>3.0.CO;2-P.

(98) Denard, C. A.; Huang, H.; Bartlett, M. J.; Lu, L.; Tan, Y.; Zhao, H.; Hartwig, J. F. Cooperative Tandem Catalysis by an Organometallic Complex and a Metalloenzyme. Angew. Chemie Int. Ed. 2014, 53 (2), 465-469. https://doi.org/10.1002/anie.201305778.

(99) Bhat, V.; Welin, E. R.; Guo, X.; Stoltz, B. M. Advances in Stereoconvergent Catalysis from 2005 to 2015: Transition-Metal-Mediated Stereoablative Reactions, Dynamic Kinetic Resolutions, and Dynamic Kinetic Asymmetric Transformations. Chem. Rev. 2017, 117 (5), 4528-4561. https://doi.org/10.1021/acs.chemrev.6b00731.

(100) Verho, O.; Bäckvall, J. E. Chemoenzymatic Dynamic Kinetic Resolution: A Powerful Tool for the Preparation of Enantiomerically Pure Alcohols and Amines. J. Am. Chem. Soc. 2015, 137 (12), 3996-4009. https://doi.org/10.1021/jacs.5b01031.

(101) Pàmies, O.; Bäckvall, J. E. Combination of Enzymes and Metal Catalysts. A Powerful Approach in Asymmetric Catalysis. Chem. Rev. 2003, 103 (8), 3247-3261. https://doi.org/10.1021/cr020029g.

(102) Chen, Z.; Aota, Y.; Nguyen, H. M. H.; Dong, V. M. Dynamic Kinetic Resolution of Aldehydes by Hydroacylation. Angew. Chemie Int. Ed. 2019, 58 (14), 4705-4709. https://doi.org/10.1002/anie.201900545.

(103) Litman, Z. C.; Wang, Y.; Zhao, H.; Hartwig, J. F. Cooperative Asymmetric Reactions Combining Photocatalysis and Enzymatic Catalysis. Nature 2018, 560 (7718), 355-359. 
https://doi.org/10.1038/s41586-018-0413-7.

(104) Atesin, A. C.; Ray, N. A.; Stair, P. C.; Marks, T. J. Etheric C-O Bond Hydrogenolysis Using a Tandem Lanthanide Triflate/Supported Palladium Nanoparticle Catalyst System. J. Am. Chem. Soc. 2012, 134 (36), 14682-14685. https://doi.org/10.1021/ja306309u.

(105) Lohr, T. L.; Li, Z.; Marks, T. J. Thermodynamic Strategies for C-O Bond Formation and Cleavage via Tandem Catalysis. Acc. Chem. Res. 2016, 49 (5), 824-834. https://doi.org/10.1021/acs.accounts.6b00069.

(106) Panteleev, J.; Zhang, L.; Lautens, M. Domino Rhodium-Catalyzed Alkyne Arylation/Palladium-Catalyzed N Arylation: A Mechanistic Investigation. Angew. Chemie Int. Ed. 2011, 50 (39), 9089-9092. https://doi.org/10.1002/anie.201103692.

(107) Friedman, A. A.; Panteleev, J.; Tsoung, J.; Huynh, V.; Lautens, M. Rh/Pd Catalysis with Chiral and Achiral Ligands: Domino Synthesis of Aza-Dihydrodibenzoxepines. Angew. Chemie Int. Ed. 2013, 52 (37), 9755-9758. https://doi.org/10.1002/anie.201303659.

(108) Zhang, L.; Qureshi, Z.; Sonaglia, L.; Lautens, M. Sequential Rhodium/Palladium Catalysis: Enantioselective Formation of Dihydroquinolinones in the Presence of Achiral and Chiral Ligands. Angew. Chemie Int. Ed. 2014, 53 (50), 13850-13853. https://doi.org/10.1002/anie.201407400.

(109) Huang, H.; Denard, C. A.; Alamillo, R.; Crisci, A. J.; Miao, Y.; Dumesic, J. A.; Scott, S. L.; Zhao, H. Tandem Catalytic Conversion of Glucose to 5-Hydroxymethylfurfural with an Immobilized Enzyme and a Solid Acid. ACS Catal. 2014, 4 (7), 2165-2168. https://doi.org/10.1021/cs500591f. 
(110) Cybulski, O.; Dygas, M.; Mikulak-Klucznik, B.; Siek, M.; Klucznik, T.; Choi, S. Y.; Mitchell, R. J.; Sobolev, Y. I.; Grzybowski, B. A. Concentric Liquid Reactors for Chemical Synthesis and Separation. Nature 2020, 586 (7827), 57-63. https://doi.org/10.1038/s41586020-2768-9.

(111) Sato, H.; Hummel, W.; Gröger, H. Cooperative Catalysis of Noncompatible Catalysts through Compartmentalization: Wacker Oxidation and Enzymatic Reduction in a One-Pot Process in Aqueous Media. Angew. Chemie Int. Ed. 2015, 54 (15), 4488-4492. https://doi.org/10.1002/anie.201409590.

(112) Huff, C. A.; Sanford, M. S. Cascade Catalysis for the Homogeneous Hydrogenation of CO 2 to Methanol. J. Am. Chem. Soc. 2011, 133 (45), 18122-18125. https://doi.org/10.1021/ja208760j.

(113) Lu, J.; Dimroth, J.; Weck, M. Compartmentalization of Incompatible Catalytic Transformations for Tandem Catalysis. J. Am. Chem. Soc. 2015, 137 (40), 12984-12989. https://doi.org/10.1021/jacs.5b07257.

(114) Wheeldon, I.; Minteer, S. D.; Banta, S.; Barton, S. C.; Atanassov, P.; Sigman, M. Substrate Channelling as an Approach to Cascade Reactions. Nat. Chem. 2016, 8 (4), 299-309. https://doi.org/10.1038/nchem.2459.

(115) Bornscheuer, U. T.; Huisman, G. W.; Kazlauskas, R. J.; Lutz, S.; Moore, J. C.; Robins, K. Engineering the Third Wave of Biocatalysis. Nature 2012, 485 (7397), 185-194. https://doi.org/10.1038/nature11117.

(116) Hyster, T. K.; Ward, T. R. Genetic Optimization of Metalloenzymes: Enhancing Enzymes 
for Non-Natural Reactions. Angew. Chemie Int. Ed. 2016, 55 (26), 7344-7357. https://doi.org/10.1002/anie.201508816.

(117) Dydio, P.; Ploeger, M.; Reek, J. N. H. Selective Isomerization-Hydroformylation Sequence: A Strategy to Valuable $\alpha$-Methyl-Branched Aldehydes from Terminal Olefins. ACS Catal. 2013, 3 (12), 2939-2942. https://doi.org/10.1021/cs400872a.

(118) Yin, X.-P.; Zeng, X.-P.; Liu, Y.-L.; Liao, F.-M.; Yu, J.-S.; Zhou, F.; Zhou, J. Asymmetric Triple Relay Catalysis: Enantioselective Synthesis of Spirocyclic Indolines through a OnePot Process Featuring an Asymmetric 6 $\pi$ Electrocyclization. Angew. Chemie Int. Ed. 2014, 53 (50), 13740-13745. https://doi.org/10.1002/anie.201407677.

(119) Dhiman, S.; Mishra, U. K.; Ramasastry, S. S. V. One-Pot Trimetallic Relay Catalysis: A Unified Approach for the Synthesis of $\beta$-Carbolines and Other [ c ]-Fused Pyridines. Angew. Chemie Int. Ed. 2016, 55 (27), 7737-7741. https://doi.org/10.1002/anie.201600840.

(120) Casnati, A.; Lichosyt, D.; Lainer, B.; Veth, L. .; Dydio, P. Multi-Catalytic Approach to OnePot Stereoselective Synthesis of Secondary Benzylic Alcohols. ChemRxiv 2021. https://doi.org/https://doi.org/10.26434/chemrxiv.14115551.v1.

(121) Camp, J. E. Auto-Tandem Catalysis: Activation of Multiple, Mechanistically Distinct Process by a Single Catalyst. European J. Org. Chem. 2017, 2017 (3), 425-433. https://doi.org/10.1002/ejoc.201600803.

(122) Shindoh, N.; Takemoto, Y.; Takasu, K. Auto-Tandem Catalysis: A Single Catalyst Activating Mechanistically Distinct Reactions in a Single Reactor. Chem. - A Eur. J. 2009, 15 (45), 12168-12179. https://doi.org/10.1002/chem.200901486. 
(123) Kanbayashi, N.; Takenaka, K.; Okamura, T.; Onitsuka, K. Asymmetric Auto-Tandem Catalysis with a Planar-Chiral Ruthenium Complex: Sequential Allylic Amidation and Atom-Transfer Radical Cyclization. Angew. Chemie Int. Ed. 2013, 52 (18), 4897-4901. https://doi.org/10.1002/anie.201300485.

(124) Matsushima, Y.; Onitsuka, K.; Kondo, T.; Mitsudo, T.; Takahashi, S. Asymmetric Catalysis of Planar-Chiral Cyclopentadienylruthenium Complexes in Allylic Amination and Alkylation. J. Am. Chem. Soc. 2001, 123 (42), 10405-10406. https://doi.org/10.1021/ja0163341.

(125) Long, J.; Yu, R.; Gao, J.; Fang, X. Access to 1,3-Dinitriles by Enantioselective Auto-tandem Catalysis: Merging Allylic Cyanation with Asymmetric Hydrocyanation. Angew. Chemie Int. Ed. 2020, 59 (17), 6785-6789. https://doi.org/10.1002/anie.202000704.

(126) Wu, X.; Cruz, F. A.; Lu, A.; Dong, V. M. Tandem Catalysis: Transforming Alcohols to Alkenes by Oxidative Dehydroxymethylation. J. Am. Chem. Soc. 2018, 140 (32), 1012610130. https://doi.org/10.1021/jacs.8b06069.

(127) Lecomte, M.; Lipshultz, J. M.; Kim-Lee, S.-H.; Li, G.; Radosevich, A. T. Driving Recursive Dehydration by P III /P V Catalysis: Annulation of Amines and Carboxylic Acids by Sequential C-N and C-C Bond Formation. J. Am. Chem. Soc. 2019, 141 (32), 1250712512. https://doi.org/10.1021/jacs.9b06277.

(128) Zhang, S.; del Pozo, J.; Romiti, F.; Mu, Y.; Torker, S.; Hoveyda, A. H. Delayed Catalyst Function Enables Direct Enantioselective Conversion of Nitriles to NH 2 -Amines. Science 2019, 364 (6435), 45-51. https://doi.org/10.1126/science.aaw4029. 
(129) Li, L.; Herzon, S. B. Temporal Separation of Catalytic Activities Allows Anti-Markovnikov Reductive Functionalization of Terminal Alkynes. Nat. Chem. 2014, 6 (1), 22-27. https://doi.org/10.1038/nchem.1799.

(130) Korvorapun, K.; Kaplaneris, N.; Rogge, T.; Warratz, S.; Stückl, A. C.; Ackermann, L. Sequential Meta-/Ortho-C-H Functionalizations by One-Pot Ruthenium(II/III) Catalysis. ACS Catal. 2018, 8 (2), 886-892. https://doi.org/10.1021/acscatal.7b03648.

(131) Semwal, S.; Choudhury, J. Switch in Catalyst State: Single Bifunctional Bi-State Catalyst for Two Different Reactions. Angew. Chemie Int. Ed. 2017, 56 (20), 5556-5560. https://doi.org/10.1002/anie.201702142.

(132) Meng, J.; Fan, L.-F.; Han, Z.-Y.; Gong, L.-Z. $\alpha$-Quaternary Chiral Aldehydes from Styrenes, Allylic Alcohols, and Syngas via Multi-Catalyst Relay Catalysis. Chem 2018, 4 (5), 1047-1058. https://doi.org/10.1016/j.chempr.2018.03.010.

(133) Wu, H.; He, Y.-P.; Gong, L.-Z. The Combination of Relay and Cooperative Catalysis with a Gold/Palladium/Brønsted Acid Ternary System for the Cascade Hydroamination/Allylic Alkylation Reaction. Adv. Synth. Catal. 2012, 354 (6), 975-980. https://doi.org/10.1002/adsc.201100922.

(134) Wang, C.; Han, Z.-Y.; Luo, H.-W.; Gong, L.-Z. Highly Enantioselective Relay Catalysis in the Three-Component Reaction for Direct Construction of Structurally Complex Heterocycles. Org. Lett. 2010, 12 (10), 2266-2269. https://doi.org/10.1021/ol1006086.

(135) Pirnot, M. T.; Rankic, D. A.; Martin, D. B. C.; MacMillan, D. W. C. Photoredox Activation for the Direct -Arylation of Ketones and Aldehydes. Science 2013, 339 (6127), 1593-1596. 
https://doi.org/10.1126/science.1232993.

(136) DeHovitz, J. S.; Loh, Y. Y.; Kautzky, J. A.; Nagao, K.; Meichan, A. J.; Yamauchi, M.; MacMillan, D. W. C.; Hyster, T. K. Static to Inducibly Dynamic Stereocontrol: The Convergent Use of Racemic $\beta$-Substituted Ketones. Science 2020, 369 (6507), 1113-1118. https://doi.org/10.1126/science.abc9909.

(137) Fleischer, S.; Werkmeister, S.; Zhou, S.; Junge, K.; Beller, M. Consecutive Intermolecular Reductive Hydroamination: Cooperative Transition-Metal and Chiral Brønsted Acid Catalysis. Chem. - A Eur. J. 2012, 18 (29), 9005-9010. https://doi.org/10.1002/chem.201200109.

(138) Tang, X.; Gan, L.; Zhang, X.; Huang, Z. N-Alkanes to n -Alcohols: Formal Primary C-H Bond Hydroxymethylation via Quadruple Relay Catalysis. Sci. $A d v$. 2020, 6 (47), eabc6688. https://doi.org/10.1126/sciadv.abc6688.

(139) Domański, S.; Gatlik, B.; Chaładaj, W. Pd-Catalyzed Boroperfluoroalkylation of Alkynes Opens a Route to One-Pot Reductive Carboperfluoroalkylation of Alkynes with Perfluoroalkyl and Aryl Iodides. Org. Lett. 2019, 21 (13), 5021-5025. https://doi.org/10.1021/acs.orglett.9b01618.

(140) Yamamoto, K.; Bruun, T.; Kim, J. Y.; Zhang, L.; Lautens, M. A New Multicomponent Multicatalyst Reaction (MC) 2 R: Chemoselective Cycloaddition and Latent Catalyst Activation for the Synthesis of Fully Substituted 1,2,3-Triazoles. Org. Lett. 2016, 18 (11), 2644-2647. https://doi.org/10.1021/acs.orglett.6b00975.

(141) Armstrong, M. K.; Goodstein, M. B.; Lalic, G. Diastereodivergent Reductive Cross 
Coupling of Alkynes through Tandem Catalysis: Z - and E -Selective Hydroarylation of Terminal Alkynes. J. Am. Chem. Soc. 2018, 140 (32), 10233-10241. https://doi.org/10.1021/jacs.8b05113. 


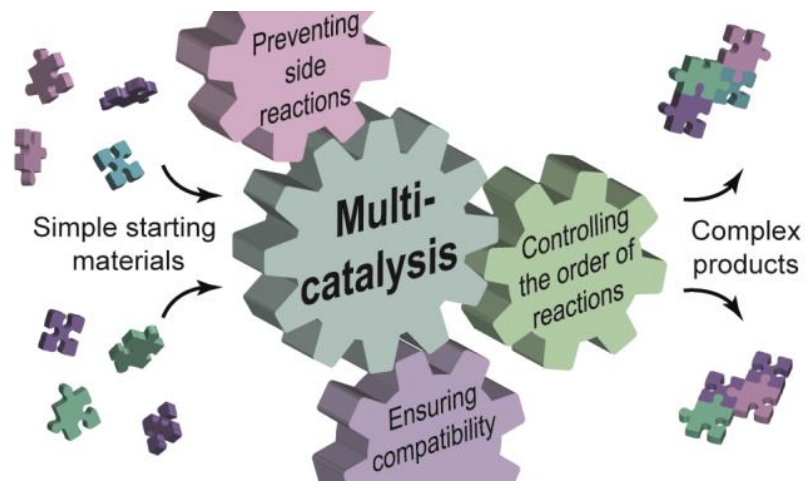

